\title{
Topological study of diverse hydrogen-bonded patterns found in a system of a nickel(II) complex and the sulfate anion
}

\section{Miguel Angel Harvey, Sebastián Suarez, Pavel N. Zolotarev, Davide M. Proserpio and Ricardo Baggio}

Acta Cryst. (2018). C74, 351-359

\section{IIUCr Journals CRYSTALLOGRAPHY JOURNALS ONLINE \\ Copyright (C) International Union of Crystallography \\ Author(s) of this paper may load this reprint on their own web site or institutional repository provided that this cover page is retained. Republication of this article or its storage in electronic databases other than as specified above is not permitted without prior permission in writing from the IUCr. \\ For further information see http://journals.iucr.org/services/authorrights.html}


STRUCTURAL

CHEMISTRY

ISSN 2053-2296

Received 19 January 2018

Accepted 9 February 2018

Edited by A. L. Spek, Utrecht University, The Netherlands

Keywords: hydrogen-bonded porous structures; crystal structure; sulfate bridge; topological analysis.

CCDC references: $1823175 ; 1823174$; 1823173

Supporting information: this article has supporting information at journals.iucr.org/c

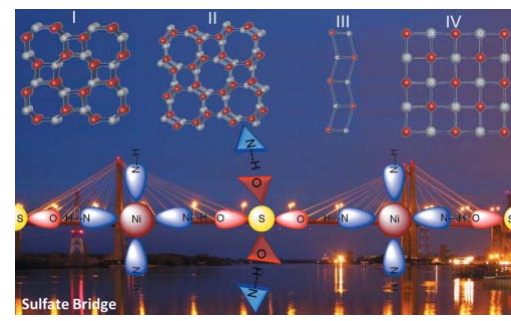

(C) 2018 International Union of Crystallography

\section{Topological study of diverse hydrogen-bonded patterns found in a system of a nickel(II) complex and the sulfate anion}

\author{
Miguel Angel Harvey, ${ }^{\mathrm{a}, \mathrm{b} *}$ Sebastián Suarez, ${ }^{\mathrm{c}}$ Pavel N. Zolotarev, ${ }^{\mathrm{d}}$ Davide M. \\ Proserpio $^{\mathrm{d}, \mathrm{e}}$ and Ricardo Baggio ${ }^{\mathrm{f}}$ *
}

\begin{abstract}
a Universidad Nacional de la Patagonia, Sede Trelew, 9100 Trelew, Chubut, Argentina, ${ }^{\mathbf{b} C e n P a t, C O N I C E T,} 9120$ Puerto Madryn, Chubut, Argentina, ' Departamento de Química Inorgánica, Analítica y Química, Física/INQUIMAE-CONICET, Facultad de Ciencias Exactas y Naturales, Universidad de Buenos Aires, Buenos Aires, Argentina, ${ }^{\mathbf{d}}$ Samara Center for Theoretical Materials Science (SCTMS), Samara State Technical University, Molodogvardeyskaya St. 244, 443100 Samara, Russian Federation, ${ }^{\mathbf{e}}$ Dipartimento di Chimica, Università degli Studi di Milano, Via C. Golgi 19, 20133 Milano, Italy, and ${ }^{\mathfrak{f} G e r e n c i a ~ d e ~ I n v e s t i g a c i o ́ n ~ y ~ A p l i c a c i o n e s, ~ C e n t r o ~ A t o ́ m i c o ~ C o n s t i t u y e n t e s, ~ C o m i s i o ́ n ~ N a c i o n a l ~ d e ~ E n e r g i ́ a ~ A t o ́ m i c a, ~}$ Buenos Aires, Argentina. *Correspondence e-mail: lauaye@gmail.com, baggio@tandar.cnea.gov.ar
\end{abstract}

A nickel(II) coordination complex, bis[2,6-bis $\left(1 H\right.$-benzimidazol-2-yl- $\left.\kappa N^{3}\right)$ pyridine- $\kappa N]$ nickel(II) sulfate, $\left[\mathrm{Ni}\left(\mathrm{C}_{19} \mathrm{H}_{13} \mathrm{~N}_{5}\right)_{2}\right] \mathrm{SO}_{4}$ or $\left[\mathrm{Ni}\left(\mathrm{H}_{2} L\right)_{2}\right] \mathrm{SO}_{4}$, having four peripheral tetrahedrally oriented $\mathrm{N}-\mathrm{H}$ donor units, combines with sulfate bridges to create hydrogen-bonded structures of varied dimensionality. The three crystal structures reported herein in the space groups $P 2_{1} 2_{1} 2_{1}, I \overline{4}$ and $P c c n$ are defined solely by strong charge-assisted $\mathrm{N}-\mathrm{H} \cdots \mathrm{O}$ hydrogen bonds and contain disordered guests (water and dimethylformamide) that vary in size, shape and degree of hydrophilicity. Two of the compounds are channelled solids with three-dimensional structures, while the third is one-dimensional in nature. In spite of their differences, all three present a striking resemblance to the previously reported anhydrous relative [Guo et al. (2011). Chin. J. Inorg. Chem. 27, 1517-1520], which is considered as the reference framework from which all three title compounds are derived. The hydrogen-bonded frameworks are described and compared using crystallographic and topological approaches.

\section{Introduction}

In the process of looking for adequate metal-organic nodes for hydrogen-bonded frameworks, we came across a family of complexes we had worked with some time ago (Harvey et al., 2003, 2013), formulated as $M\left(\mathrm{H}_{2} L\right)_{2}\left[\mathrm{H}_{2} L\right.$ is 2,6-bis $(1 H$ benzimidazol-2-yl)pyridine and $M$ is a transition metal], where the two identical $\mathrm{H}_{2} L$ ligands bind the metal in an orthogonal fashion relative to each other, giving the bulky $M\left(\mathrm{H}_{2} L\right)_{2}$ group a 'pseudo-spherical' shape, with four active $\mathrm{N}-\mathrm{H}$ donor groups pointing outwards in an approximate tetrahedral geometry around the metal atom (Fig. 1a). The bulky group looked promising as a 'tetrahedral hydrogen-bonding donor' acting as a nodal centre in an eventual hydrogen-bond network (Fig. 1b), in which case, a linker could be chosen with similar acceptor capabilities which are able to expand by selforganization into a porous three-dimensional array. The simplest example we could think of was sulfate $\left(\mathrm{SO}_{4}{ }^{2-}\right)$, with a similar tetrahedral distribution of hydrogen-bonding acceptors. The feasibility of this approach was assessed with respect to other complex structures already reported in the literature, $v i z$. the interpenetrating network in a hydrogen-bonded chromium sulfate biimidazolate described by Larsson \& Öhrström (2003), and we decided to attempt the synthesis of the corresponding $M\left(\mathrm{H}_{2} L\right)_{2}$ sulfates in the hope of obtaining 
some kind of 'diamond-like' hydrogen-bonded structure. Investigation of the $M\left(\mathrm{H}_{2} L\right)_{2} \mathrm{SO}_{4}$ system proved fruitful in that it provided a number of compounds, not only with the expected geometry, as hydrogen-bonded porous networks, but also in their properties to fine tune their geometry by adequately choosing the solvates, which end up acting as efficient crystallization templates. We report herein the results of these attempts with $\mathrm{Ni}^{2+}$ as the cation and dimethylformamide/water as the solvents.

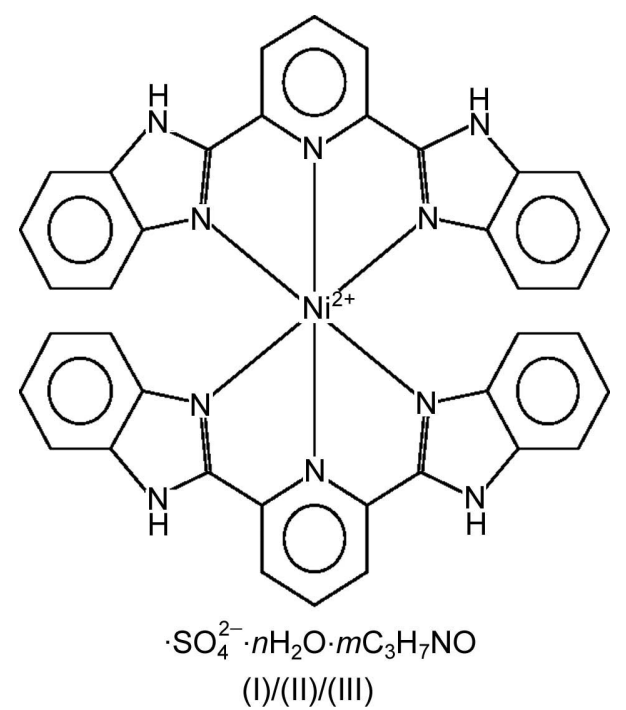

The three phases of bis[2,6-bis(1H-benzimidazol-2-yl- $\left.\kappa N^{3}\right)$ pyridine- $\kappa N]$ nickel(II) sulfate reported herein (see Scheme), with space groups $P 2_{1} 2_{1} 2_{1}$ for (I), $I \overline{4}$ for (II) and $P c c n$ for (III), are formulated as $\left[\mathrm{Ni}\left(\mathrm{H}_{2} L\right)_{2}\right] \mathrm{SO}_{4} \cdot n \mathrm{H}_{2} \mathrm{O} \cdot m \mathrm{DMF}$ (DMF is dimethylformamide), with $n$ and $m$ undeterminable by conventional X-ray diffraction (XRD) methods (see Refinement section for details). A search in the Cambridge Structural Database (CSD, Version 5.37 and updates; Groom et al., 2016) for pre-existing members of the $\left[\mathrm{Ni}\left(\mathrm{H}_{2} L\right)_{2}\right]^{2+}\left(\mathrm{SO}_{4}\right)^{2-}$ family resulted in only an unsolvated member [Guo et al., 2011; CSD refcode OYAKEF, hereinafter (IV)], which will be included as a reference in our discussion.

\section{Experimental}

\subsection{Synthesis and crystallization}

All reagents used were commercially available and used without further purification. Compound (I) was obtained by dissolution of $\mathrm{NiSO}_{4} \cdot 6 \mathrm{H}_{2} \mathrm{O}$ (CAS Number 10101-97-0, SigmaAldrich) and $\mathrm{H}_{2} L$ (CAS Number 28020-73-7, Sigma-Aldrich) in $\mathrm{DMF}$ in a 1:2 molar ratio up to a final concentration of $0.010 M$ of the $\mathrm{Ni}^{2+}$ cation. Compounds (II) and (III) were synthesized in a similar fashion, by mixing equal volumes of equimolar solutions $(0.010 M)$ of $\mathrm{NiSO}_{4} \cdot 6 \mathrm{H}_{2} \mathrm{O}$ in water and $\mathrm{H}_{2} L$ in DMF. Upon mixing, the immediate precipitation of a preliminary poorly crystallized phase occurred, which was discarded. After a couple of months of unperturbed evaporation, pale-red columnar crystals pertaining to some of the reported phases were obtained, within a mixture of further untargeted products. The acquisition of one particular phase could not so far be adequately controlled, its appearance being dependent on the solvent quality/ratio and crystallization conditions.

\subsection{Refinement}

All three title structures could be solved in a conventional way (SHELXS97; Sheldrick, 2008), but refinement (SHELXL2016; Sheldrick, 2015) posed some problems derived from the fact that the structures showed large solventaccessible voids of 29,46 and $7 \mathrm{vol} \%$ and any further improvement in the refinement was jeopardized by the disordered water/DMF solvent molecules delocalized in these regions. A direct consequence of this was the impossibility of refining (even with restraints) the active $\mathrm{H}$ atoms in the structures, which had to be treated, as had the rest, in the riding approximation $[\mathrm{N}-\mathrm{H}=0.86 \AA$ and $\mathrm{C}-\mathrm{H}=0.93 \AA$, with $U_{\text {iso }}(\mathrm{H})=1.2 U_{\text {eq }}$ (host)]. In view of these characteristics, the refinements were treated under the 'partial structure factors' algorithm implemented as the SQUEEZE option (Spek, 2015) in the PLATON package (Spek, 2009), which allowed for reasonable structural descriptions and reported $R$ factors of 0.074 for (I), 0.078 for (II) and 0.086 for (III). For the non-H atoms, rigid-bond restraints were applied to the anisotropic displacement parameters of bonded (1,2-) and nonbonded (1,3-) atom pairs. In addition, similarity restraints were applied to the geometries of the two independent organic ligands of the cation in each structure.

\subsection{Topological analysis}

Topological analysis was performed using the ToposPro program package (Blatov et al., 2014). The determination of all intermolecular interactions was carried out by means of the recently proposed 'domains method' (Blatov, 2016) that is based on Voronoi partition and implemented in the AutoCN program, which is a part of the ToposPro code. Among intermolecular interactions, the $D-\mathrm{H} \cdots A$ hydrogen bonds in a fragment are identified in accordance with the following additional geometrical criteria: $d(\mathrm{H} \cdots A)<2.5 \AA, d(D \cdots A)<$ $3.5 \AA$ and $\angle D-\mathrm{H} \cdots A>120^{\circ}(D=\mathrm{N}, \mathrm{O} ; A=\mathrm{N}, \mathrm{O}, \mathrm{F}, \mathrm{S}, \mathrm{Cl})$. The underlying nets of the investigated structures, which represent the overall connectivity of molecules in hydrogenbonded networks, were obtained after the simplification procedure performed in the $A D S$ program. This includes the representation of a molecule by its centre of mass, keeping the connectivity of molecules with each other by means of hydrogen bonds; all hydrogen bonds between a given pair of molecules transform to the same edge between the molecular centres of mass in the simplified net. The TTD collection of periodic network topologies and the TTO collection containing representatives of different topological types were used to determine the topological type of underlying nets (Blatov et al., 2014). The RCSR three-letter symbols (O'Keeffe et al., 2008) and the Koch \& Fischer (1978) nomenclature for 1- or 2-periodic sphere packings are used to designate network topologies. The nets absent in the RCSR database are designated with the ToposPro NDn nomen- 
Table 1

Crystal and refinement data for (I), (II) and (III) (herein reported), and (IV) (Guo et al., 2011) (included for comparison).

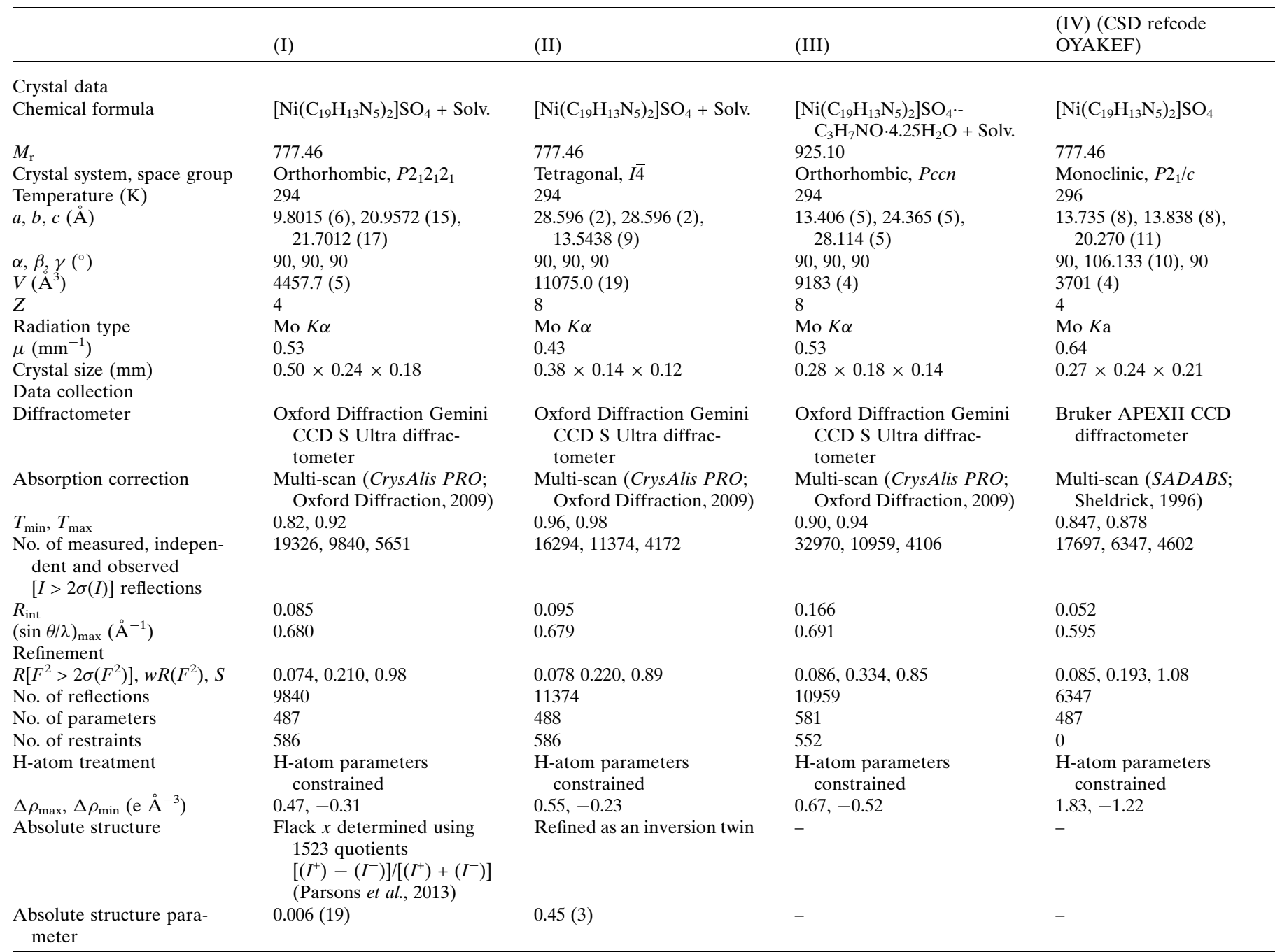

Computer programs: CrysAlis PRO (Oxford Diffraction, 2009), SHELXS97 (Sheldrick, 2008), SHELXL2016 (Sheldrick, 2015), SHELXTL (Sheldrick, 2008), SHELXL2014 (Sheldrick, 2015) and PLATON (Spek, 2009).

clature (Alexandrov et al., 2011), where $\mathrm{N}$ is a sequence of coordination numbers of all non-equivalent nodes of the net, $\mathrm{D}$ is the periodicity of the net $(\mathrm{D}=\mathrm{M}, \mathrm{C}, \mathrm{L}$ and $\mathrm{T}$ for $0-, 1-, 2-$ and 3-periodic nets, respectively) and $\mathrm{n}$ is the ordinal number of the net in the set of all non-isomorphic nets with the given ND sequence. The local connectivity of the molecules was described by means of the molecular connection type symbol (MCTS) $\mathrm{L}^{\text {mbtkpghond }}$ proposed in Aman et al. (2014) for the description of intermolecular connectivity in hydrogenbonded networks. Each molecule (L) is designated by superscripts $\mathrm{M}, \mathrm{B}, \mathrm{T}, \mathrm{K}, \mathrm{P}, \mathrm{G}, \mathrm{H}, \mathrm{O}, \mathrm{N}$ and $\mathrm{D}$ depending on the number $\mathrm{n}=1-10$ of its atoms (both hydrogen-bond donors and acceptors) involved in the formation of intermolecular hydrogen bonds. The total number of molecules connected to a given molecule is listed as the upper index in the line mbtkpghond, where each integer $\mathrm{m}, \mathrm{b}, \mathrm{t}, \mathrm{k}$, etc., corresponds to the number of molecules connected by one, two, three, four, etc., hydrogen bonds. Additionally, a recent inclusion in the ToposPro package was applied to evaluate the coordination modes of both the complex cation and the sulfate anion. This algorithm compares the similarity of the coordination figure of an atom or a molecule to some idealized coordination figures on the basis of comparison of angular fingerprints of coordination polyhedra (Shevchenko et al., 2017). In these calculations, parameters $\delta$ and $\sigma$ (as defined in Shevchenko's paper) were given values of 18 and $9^{\circ}$, respectively.

\section{Results and discussion}

\subsection{Crystal structure}

Table 1 presents crystal and refinement data for (I), (II) and (III) (reported herein), and (IV) (Guo et al., 2011), included for comparison purposes. As already stated, the structures consist of $\left[\mathrm{Ni}\left(\mathrm{H}_{2} L\right)_{2}\right]^{2+}$ cations acting as fourfold hydrogenbond donors (in the role of nodes) and sulfate counter-anions as hydrogen-bond acceptors (acting as linkers). Figs. 1(c), 1(d) and 1(e) show displacement ellipsoid plots for (I), (II) and 


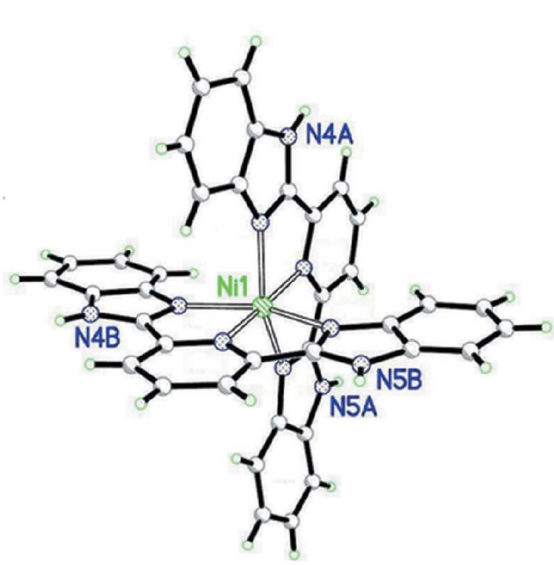

(a)

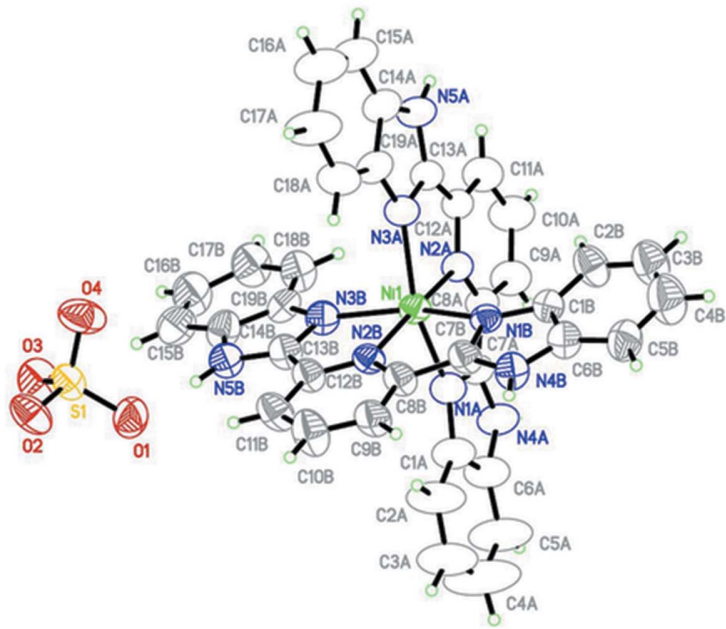

(d)

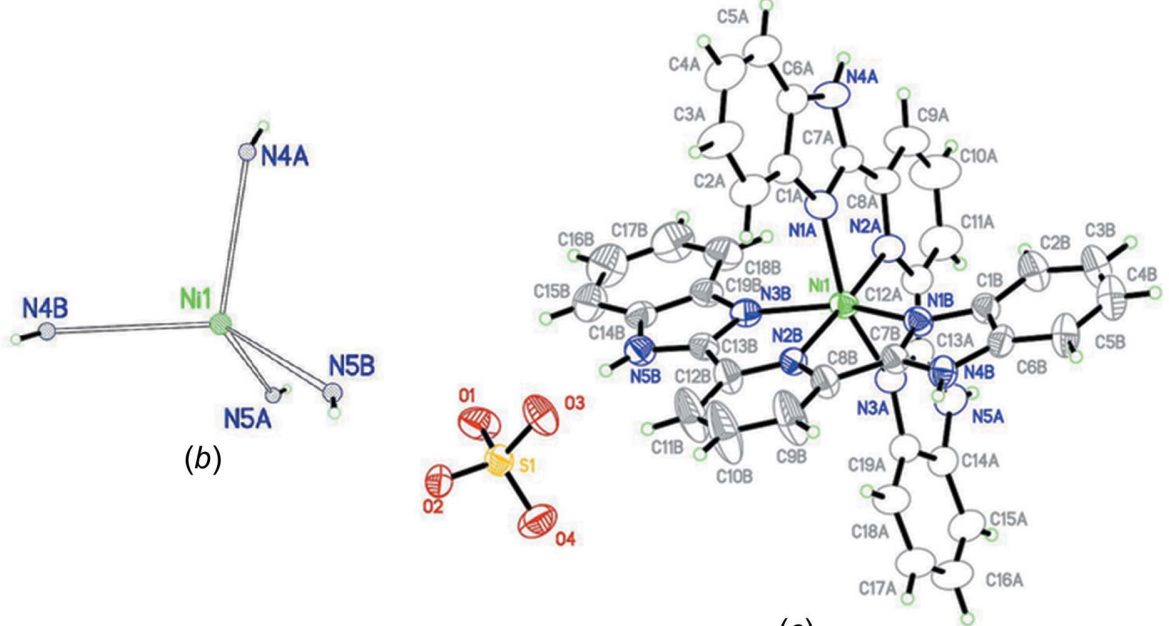

(c)

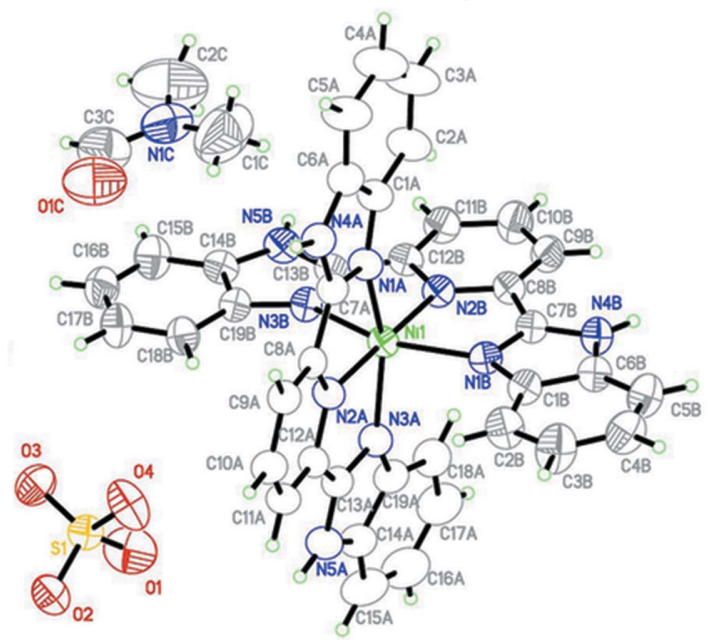

(e)

Figure 1

(a). A general $M L_{2}$ nucleus. (b) A schematic representation of panel (a) as a fourfold hydrogen-bonding donor node. $(c) /(d) /(e)$ Displacement ellipsoid plots of the asymmetric units in structures (I), (II) and (III), respectively (water molecules not shown), drawn at the $40 \%$ probability level. The $\mathrm{H}_{2} L$ ligands, at right angles to each other, have been drawn with different line shading, for clarity.

(III), respectively, showing the (common) numbering scheme used. Both the cation and the anion display the geometries usually reported in the literature and will not be discussed in particular detail since our interest will be concentrated on their hydrogen-bonding behaviour (only a comparative table of similar coordination distances is provided; Table 2). Table 3 presents the hydrogen-bonding interactions for the three structures discussed herein (where all the available $\mathrm{N}-\mathrm{H}$ groups are involved), as well as for the already reported (IV). Inspection of the table shows that in structures (I) and (II),

\footnotetext{
${ }^{1}$ At this stage, it could be argued that the hydrogen-bonding analysis, as performed herein, would be potentially incomplete due to the unresolved water/DMF solvent molecules not being taken into account, in spite of their potential capability for hydrogen bonding. An argument against this position is to be found precisely in the 'unobservance' of defined solvent molecules: hydrogen bonding to the mainframe, when present at all, has a definite 'anchoring effect', whose most inmediate result is the concentration of the corresponding electron density in a rather well-defined portion of space, thus rendering the molecule 'crystallographically visible' in a difference map. An example of this is the already discussed $\mathrm{N} 5 A-\mathrm{H} 5 A A \cdots \mathrm{O} 3 W$ hydrogen bond in structure (III), which renders the $\mathrm{O} 3 W$ solvent molecule 'detectable'.
}

each sulfate ion binds to four different $\mathrm{Ni}\left(\mathrm{H}_{2} L\right)_{2}$ units through its four $\mathrm{O}$ atoms bound to four different $\mathrm{N}-\mathrm{H}$ groups. In structure (III), instead, the sulfate anion binds to three $\mathrm{Ni}\left(\mathrm{H}_{2} L\right)_{2}$ units due to two $\mathrm{O}$ atoms (O1 and $\left.\mathrm{O} 3\right)$ being involved in a split hydrogen bond to the $\mathrm{N} 5 B-\mathrm{H} 5 \mathrm{~N} B$ group. This leaves a fourth $\mathrm{N}-\mathrm{H}$ group unbound to sulfate, but which binds to a solvent water molecule instead (N5A$\mathrm{H} 5 A A \cdots \mathrm{O} 3 W){ }^{1}$

In (I) and (II), this connectivity leads to three-dimensional structures having large channels (Fig. 2). In the case of (I), there is only one type (denoted $\mathbf{A}$ ) which runs along [100] and accounts for $30 \%$ of the total structure volume (Fig. $2 a$ ); in (II), there are two types ( $\mathbf{A}$ and $\mathbf{B}$ ), both running along [001] and accounting for as much as $45 \%$ (Fig. $2 b$ ). These volumes are in turn occupied by the disordered water/DMF solvent molecules. In (III), the result of $\mathrm{Ni}\left(\mathrm{H}_{2} L\right)_{2} \cdots \mathrm{SO}_{4}$ hydrogen bonding is a much simpler one-dimensional supramolecular structure, with interchain linkage diffusely mediated by water/ DMF solvent molecules. Fig. 3(a) displays a horizontal view of column $\mathbf{A}$ in (I), showing the lateral 'mesh' which wraps the 
Table 2

Selected coordination distances (£) for (I), (II), (III) and (IV).

\begin{tabular}{lllll}
\hline Bond & (I) & (II) & (III) & $(\mathrm{IV})$ \\
\hline Ni1-N1A & $2.108(5)$ & $2.128(7)$ & $2.118(5)$ & $2.117(6)$ \\
Ni1-N2A & $2.029(5)$ & $2.037(6)$ & $2.017(4)$ & $2.023(6)$ \\
Ni1-N3A & $2.113(5)$ & $2.115(7)$ & $2.086(5)$ & $2.128(6)$ \\
Ni1-N1B & $2.112(6)$ & $2.149(7)$ & $2.116(5)$ & $2.101(6)$ \\
Ni1-N2B & $2.022(5)$ & $2.026(6)$ & $2.021(4)$ & $1.998(6)$ \\
Ni1-N3B & $2.120(6)$ & $2.109(7)$ & $2.101(5)$ & $2.101(6)$ \\
\hline
\end{tabular}

column and which is made up of one single type of $\left[\mathrm{Ni}\left(\mathrm{H}_{2} L\right)_{2} \cdots \mathrm{SO}_{4}\right]_{3}$ loop $\left[\mathbf{A 1}, R_{6}^{6}(24)\right.$, shaded in the figure]. This loop is topologically identical to that of column $\mathbf{A}$ in (II) (loop A1) represented in Fig. 3(b). The topological similarities are also reflected in the characteristics of the columns generated: as a crude estimator, we have taken the 'diameters' of the columns, as measured by the projected distances between opposite $\mathrm{Ni}^{2+}$ cations, which gives roughly $\sim 15.5-16.0 \AA$ for (I) and $\sim 14.0 \AA$ for (II). The main difference between both structures comes from the second column, i.e. B, which is absent in (I) but present in (II) (see Fig. 2), and which shows a more complex mesh structure (Fig. 3c), presenting large $\left[\mathrm{Ni}\left(\mathrm{H}_{2} L\right)_{2} \cdots \mathrm{SO}_{4}\right]_{3}$ loops $\left[\mathbf{B 1}, R_{6}^{6}(24)\right]$ similar to $\mathbf{A 1}$ described already, combined with smaller $\left[\mathrm{Ni}\left(\mathrm{H}_{2} L\right)_{2} \cdots \mathrm{SO}_{4}\right]_{2}$ loops [B2, $\left.R_{4}^{4}(16)\right]$. This allows for a larger diameter in the column, which, with a similar estimator as before, is $\sim 20.0 \AA$. A different scenario is seen for structure (III), where the connectivity results in the [100] chains shown in projection in Fig. 4(a) and in turn interconnected by water-mediated hydrogen bonds. These interactions are hard to describe, since water $\mathrm{H}$ atoms could not be detected, but there is, however, one significant hydrogen bond fully resolved, having $\mathrm{O} 3 W$ as acceptor and $\mathrm{H} 5 \mathrm{~N} A$ as donor, and which fulfils an important role in the packing description to follow. Fig. 4(b) presents a rotated view of Fig. 4(a) along the horizontal axis, and shows the way in
Table 3

Hydrogen-bond geometry $\left(\AA{ }^{\circ},{ }^{\circ}\right)$ for (I), (II), (III) and (IV).

\begin{tabular}{llllll}
\hline Structure & $D-\mathrm{H} \cdots A$ & $D-\mathrm{H}$ & $\mathrm{H} \cdots A$ & $D \cdots A$ & $D-\mathrm{H} \cdots A$ \\
\hline (I) & $\mathrm{N} 4 A-\mathrm{H} 4 \mathrm{~N} A \cdots \mathrm{O} 2^{\mathrm{i}}$ & 0.86 & 1.82 & $2.654(8)$ & 163 \\
& $\mathrm{~N} 5 A-\mathrm{H} 5 \mathrm{~N} A \cdots \mathrm{O} 1^{\mathrm{ii}}$ & 0.86 & 1.85 & $2.701(8)$ & 171 \\
& $\mathrm{~N} 4 B-\mathrm{H} 4 \mathrm{~N} B \cdots \mathrm{O} 4^{\mathrm{iii}}$ & 0.86 & 1.85 & $2.685(9)$ & 164 \\
& $\mathrm{~N} 5 B-\mathrm{H} 5 \mathrm{~N} B \cdots \mathrm{O} 3$ & 0.86 & 1.81 & $2.663(9)$ & 173 \\
(II) & $\mathrm{N} 4 A-\mathrm{H} 4 \mathrm{~N} A \cdots \mathrm{O} 3^{\mathrm{i}}$ & 0.86 & 1.85 & $2.706(10)$ & 172 \\
& $\mathrm{~N} 5 A-\mathrm{H} 5 \mathrm{~N} A \cdots \mathrm{O} 1^{\mathrm{ii}}$ & 0.86 & 2.51 & $3.049(10)$ & 121 \\
& $\mathrm{~N} 5 A-\mathrm{H} 5 \mathrm{~N} A \cdots \mathrm{O} 2^{\mathrm{ii}}$ & 0.86 & 2.04 & $2.883(11)$ & 165 \\
& $\mathrm{~N} 4 B-\mathrm{H} 4 \mathrm{~N} B \cdots \mathrm{O} 4^{\mathrm{iii}}$ & 0.86 & 1.89 & $2.746(10)$ & 177 \\
& $\mathrm{~N} 5 B-\mathrm{H} 5 \mathrm{~N} B \cdots \mathrm{O} 1$ & 0.86 & 1.75 & $2.605(10)$ & 174 \\
(III) & $\mathrm{N} 4 A-\mathrm{H} 4 A A \cdots \mathrm{O} 4^{\mathrm{i}}$ & 0.86 & 1.89 & $2.732(7)$ & 168 \\
& $\mathrm{~N} 5 A-\mathrm{H} 5 A A \cdots \mathrm{O} 3 W$ & 0.86 & 1.97 & $2.802(7)$ & 163 \\
& $\mathrm{~N} 4 B-\mathrm{H} 4 B A \cdots \mathrm{O} 2^{\mathrm{ii}}$ & 0.86 & 1.79 & $2.646(7)$ & 175 \\
& $\mathrm{~N} 5 B-\mathrm{H} 5 B A \cdots \mathrm{O} 1^{\mathrm{iii}}$ & 0.86 & 2.29 & $3.024(8)$ & 143 \\
& $\mathrm{~N} 5 B-\mathrm{H} 5 B A \cdots \mathrm{O} 3^{\mathrm{iii}}$ & 0.86 & 2.19 & $2.959(9)$ & 149 \\
(IV) & $\mathrm{N} 4 A-\mathrm{H} 4 \mathrm{~N} A \cdots \mathrm{O} 2^{\mathrm{i}}$ & 0.86 & 2.04 & $2.7418(16)$ & 138 \\
& $\mathrm{~N} 5 A-\mathrm{H} 5 \mathrm{~N} A \cdots \mathrm{O} 3$ & 0.86 & 1.94 & $2.7966(16)$ & 176 \\
& $\mathrm{~N} 4 B-\mathrm{H} 4 \mathrm{~N} B \cdots \mathrm{O} 1^{\mathrm{ii}}$ & 0.86 & 1.82 & $2.6806(16)$ & 179 \\
& $\mathrm{~N} 5 B-\mathrm{H} 5 \mathrm{~N} B \cdots \mathrm{O} 4^{\mathrm{iii}}$ & 0.86 & 2.11 & $2.8356(17)$ & 142 \\
\hline
\end{tabular}

Symmetry codes for (I): (i) $-x+\frac{3}{2},-y, z-\frac{1}{2}$; (ii) $-x+\frac{5}{2},-y, z-\frac{1}{2}$; (iii) $x-\frac{1}{2},-y+\frac{1}{2},-z+2$. Symmetry codes for (II): (i) $y,-x+1,-z$; (ii) $-x+\frac{3}{2},-y+\frac{1}{2}, z-\frac{1}{2}$; (iii) $-x+\frac{3}{2},-y+\frac{1}{2}, z+\frac{1}{2}$. Symmetry codes for (III): (i) $-x+2,-y+1,-z+1$; (ii) $-x+\frac{3}{2}, y, z-\frac{1}{2}$; (iii) $-x+1$, $-y+1,-z+1$. Symmetry codes for (IV): (i) $y,-x+1,-z$; (ii) $-x+\frac{3}{2},-y+\frac{1}{2}, z-\frac{1}{2}$; (iii) $-x+\frac{3}{2},-y+\frac{1}{2}, z+\frac{1}{2}$.

which chains align in the (010) plane. Note the fact that only three $\mathrm{N}-\mathrm{H}$ groups are involved in chain formation (one of them in a bifurcated mode), the fourth being involved in the hydrogen bond to the $\mathrm{O} 3 \mathrm{~W}$ solvent molecule. In structure (IV) (reported in Guo et al., 2011), the hydrogen-bonded network is defined by [010] chains (viewed as closed loops in Fig. 5a), which are linked along [100] via an $\mathrm{N}-\mathrm{H} \cdot \mathrm{OSSO}_{3}$ hydrogen bond to form slightly corrugated two-dimensional arrays parallel to (001) and shown in projection as the undulating structures running top to bottom in Fig. 5(a). Fig. 5(b), in turn, presents a rotated view of Fig. 5(a), displaying these twodimensional substructures in full.

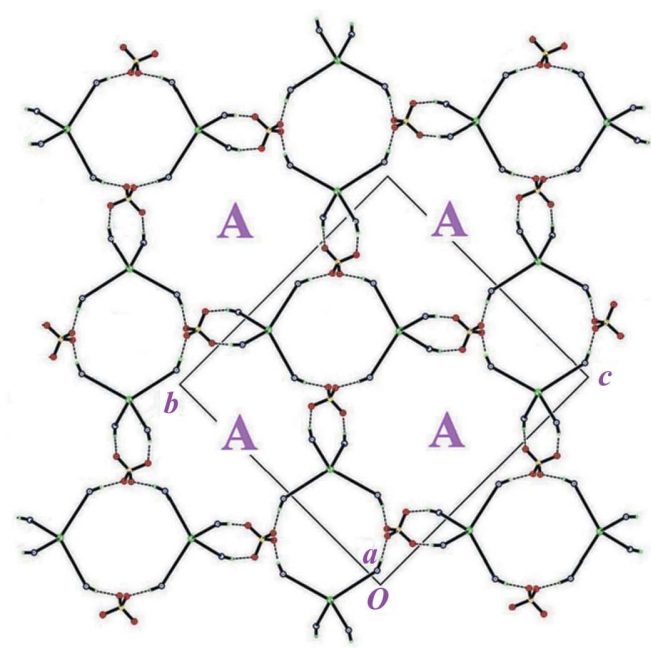

(a)

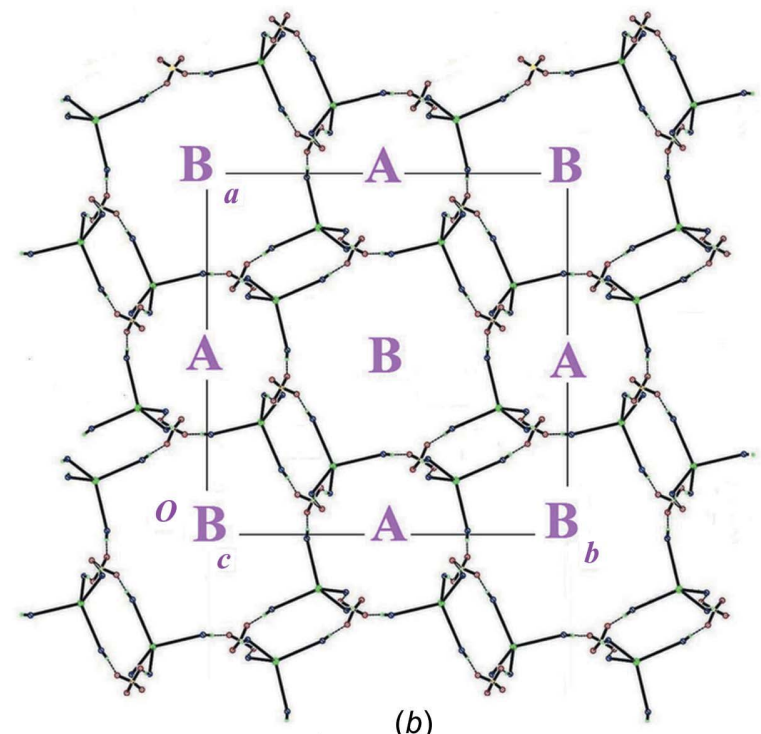

(b)

Figure 2

Schematic projections of structures (I) and (II) along the channels, showing (a) structure (I) (only channels of type A) and (b) structure (II) (channels of types $\mathbf{A}$ and $\mathbf{B}$ ). 


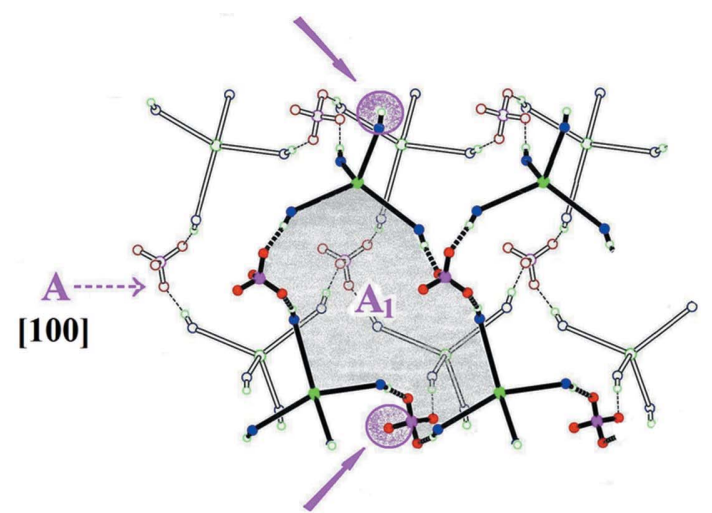

(a)

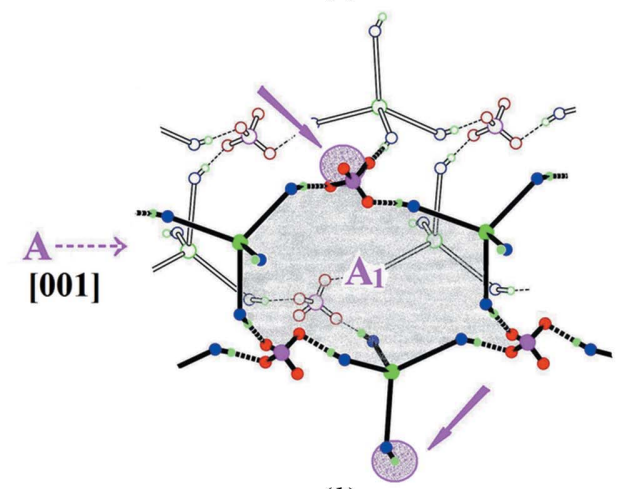

(b)

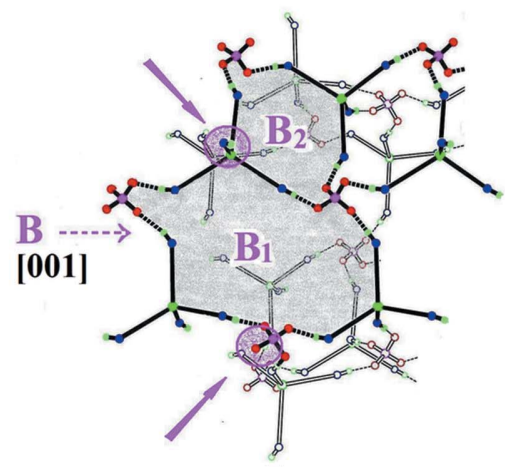

(c)

Figure 3

Schematic representation of the meshes wrapping channels (displayed horizontally) in (I) and (II) for (a) channel $\mathbf{A}$ of structure (I), showing mesh motif A1, (b) channel $\mathbf{A}$ of structure (II), showing mesh motif A1, and $(c)$ channel $\mathbf{B}$ of structure (II), showing mesh motifs $\mathbf{B 1}$ and $\mathbf{B 2}$. Broken arrows suggest the tube direction. (The full arrows are relevant to the discussion in document SD1 of the supporting information.)

A point common to all three solvatomorphs (I), (II) and (III) is the fact that they are directly related to the structure of this unsolvated 'parent' (IV), and which could be considered as the reference frame from which all three derive. A detailed analysis of the way in which this relationship is achieved is presented in document SD1 of the supporting information.

\subsection{Analysis of the topology of the hydrogen-bonded} networks

In structures (I), (II) and (IV), both the complex cation and the sulfate anion have four active centres (atoms that parti-

cipate in hydrogen bonding) and form one hydrogen bond with each of the four neighbours; therefore the connection type of both ions is $\mathrm{K}^{4}$ (see Fig. S1 in the supporting information). On the contrary, in structure (III), the ions have different local connectivities of $\mathrm{K}^{31}$ and $\mathrm{K}^{21}$ for the cation and anion, respectively. The standard representation of the hydrogen-bonded structures leads to the underlying nets shown in Fig. 6. The sql topological type of the underlying net in (IV) is the most abundant among layered hydrogen-bonded structures (Zolotarev et al., 2014), while the unc and atn topologies found in (I) and (II), respectively, are very uncommon for hydrogen-bond frameworks in molecular crystals, especially the atn net. In the TTO collection, there are 11 molecular structures with unc topology and only one with atn topology (see Table S1 in the supporting information). The hydrogen-bonded ladder in structure (III) possesses $4^{4}(0,2)$ topology, which is the second most abundant among structures with 1-periodic hydrogen-bonded systems. To explore the bonding preferences of the complex cations with composition

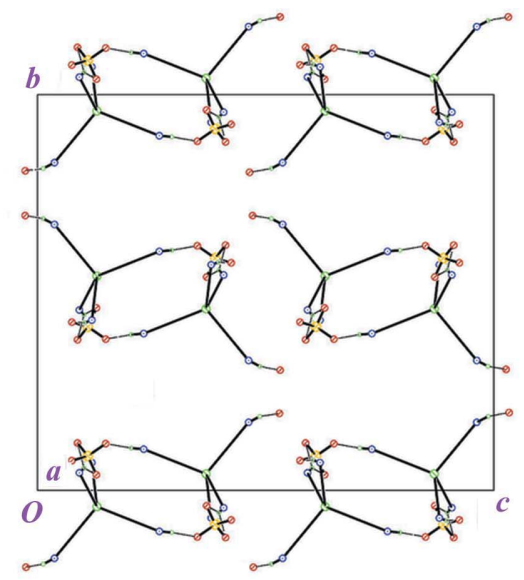

(a)

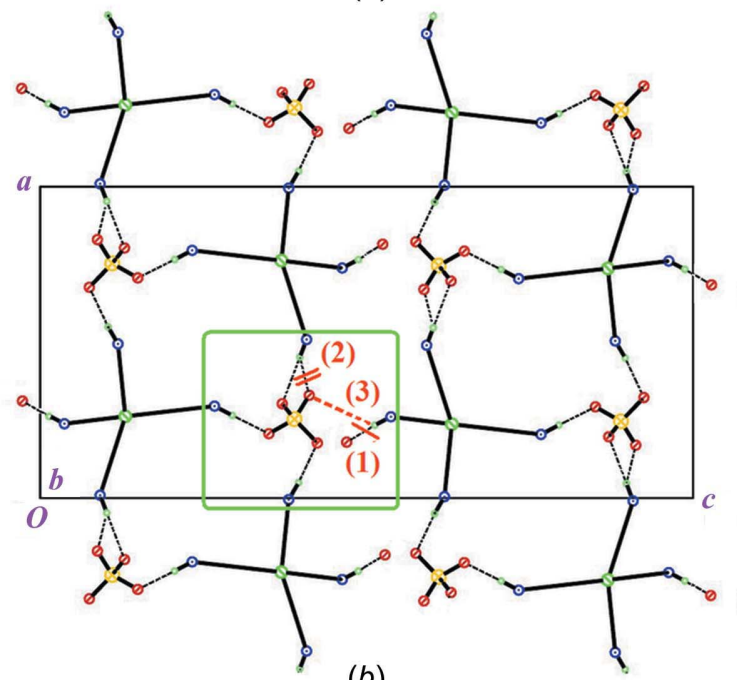

Figure 4

(b)

(a) View in projection of the [100] columnar arrays in (III) (closed loops). Note the $\mathrm{N}-\mathrm{H} \cdots \mathrm{OW}$ hydrogen bond, limiting the interconnectivity of the chains. Only the $\mathrm{O} 3 W$ solvent molecule has been considered in the analysis, for clarity. (b) A view of the (010) plane, showing (vertically) the parallel [100] columns. (The square inset is relevant to the discussion in document SD1 of the supporting information.) 


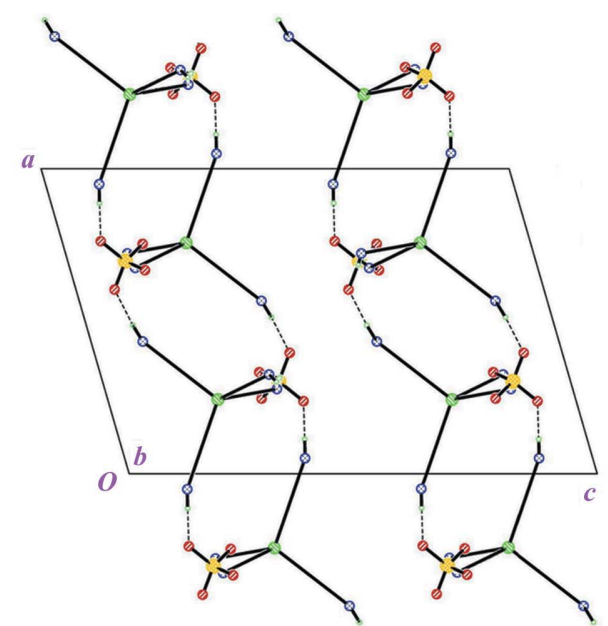

(a)

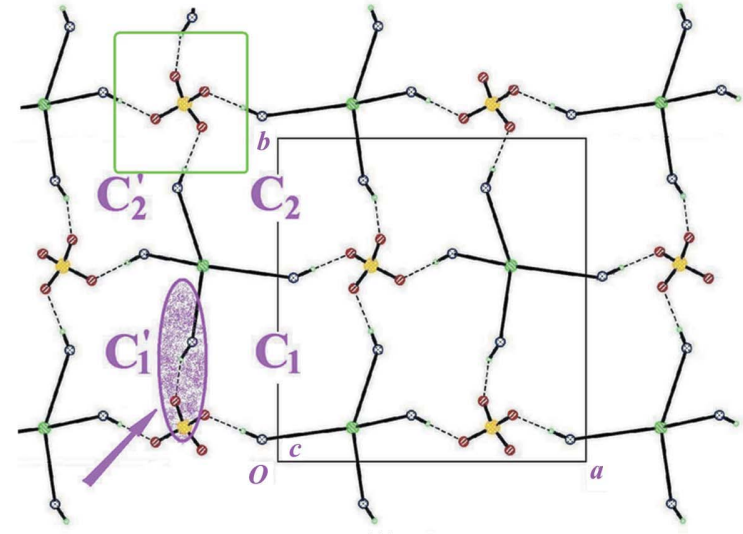

(b)

Figure 5

(a) A view in projection of the [010] columnar arrays in (IV) (closed loops), linked along [100] to form the slightly undulating (001) planes coming out of the plane of the figure. (b) One of these isolated planes, seen in full display. (The square inset, full arrow and C labels are relevant to the discussion in document SD1 of the supporting information.)

$M\left(\mathrm{H}_{2} L\right)_{2}$, we extracted from the CSD 20 crystal structures (see Table S2 in the supporting information) with the same ligand, i.e. 2,6-bis( $1 H$-benzimidazol-2-yl)pyridine, denoted $\mathrm{H}_{2} L$. From the analysis of the structures, one can assume that the connection type of such cations is usually of the $\mathrm{K}$ type (four active centres), because in all the 20 structures, it engages all $\mathrm{N}-\mathrm{H}$ groups in bonding, but for the seven rareearth-containing cations, the connectivity is greater because of the extra ligands engaged in the formation of additional hydrogen bonds. The only structure (CSD refcode EYINAB;
Harvey et al., 2004) with an anion of double negative charge, namely solvated $\mathrm{Zn}^{\mathrm{II}}-\left(\mathrm{H}_{2} L\right)_{2}$-peroxodisulfate, has the same topology as (III), i.e. $4^{4}(0,2)$, though the coordination type of the cation (MCTS $\mathrm{K}^{4}$ ) in this structure is different from that found in (III) (MCTS $\mathrm{K}^{31}$ ). With the aim of exploring the local connectivity preferences of the sulfate anion, a sample of 843 crystal structures with sulfate anions forming hydrogen bonds was extracted from the CSD by means of the ConQuest program (Bruno et al., 2002). Structures comprising both sulfate and hydrogen sulfate anions were eliminated from the

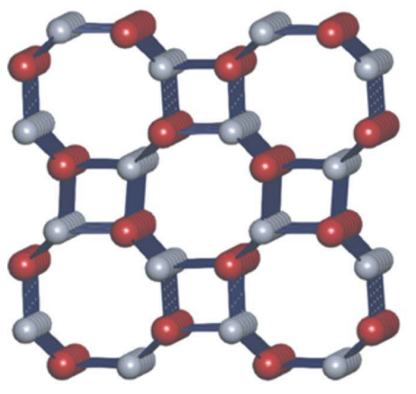

unc

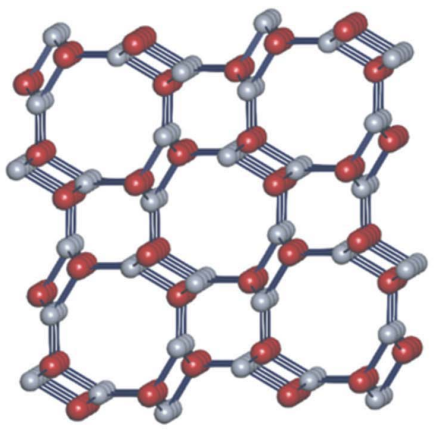

atn

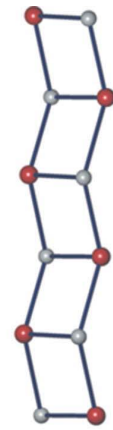

$4^{4}(0,2)$

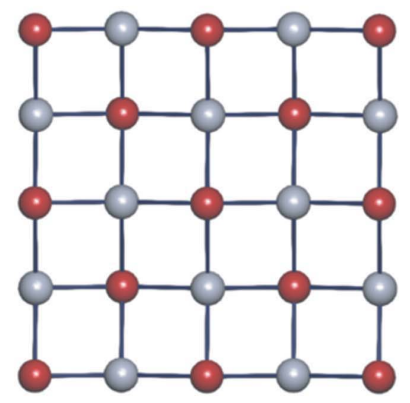

sql
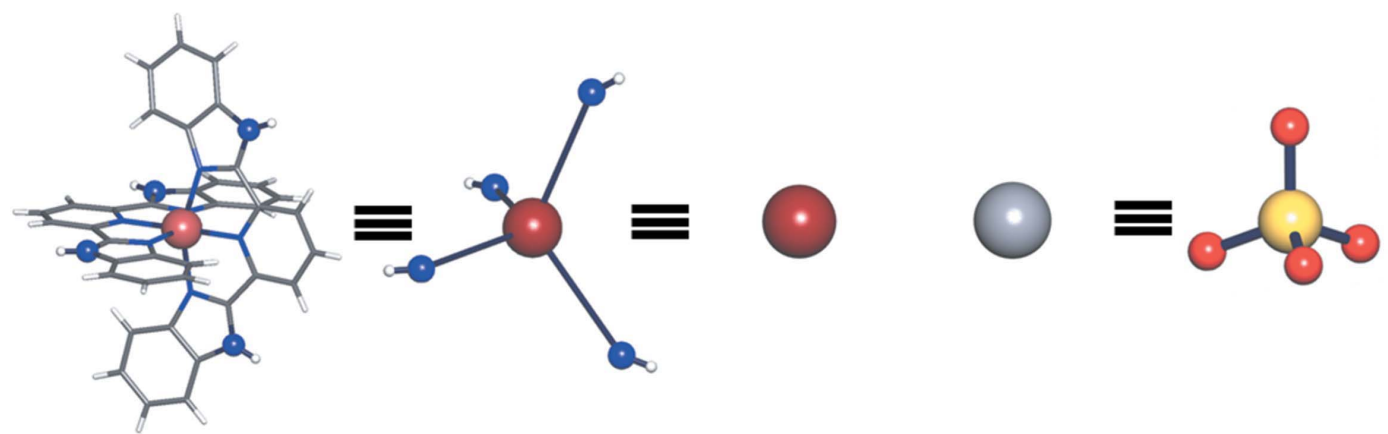

Figure 6

The underlying nets in the crystal structures of (I), (II), (III) and (IV) (left to right), with the corresponding topological type of the net underneath. The underlying net represents the connection pattern of complex cations and sulfate anions (nodes of the net) by means of hydrogen bonds (edges between nodes). The unc net is intrinsically chiral. 


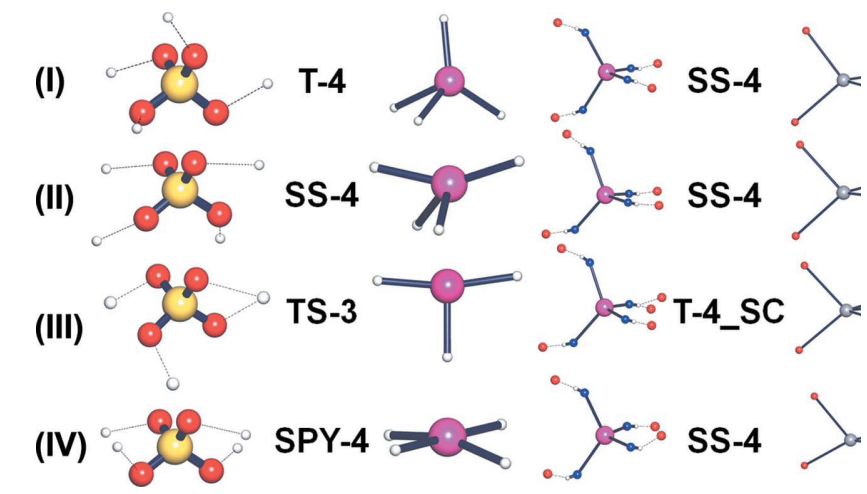

Figure 7

Hydrogen bonds formed by the molecular ions in structures (I)-(IV), along with the resulting coordination modes: T-4 is a tetrahedron, SS-4 is a see-saw, TS-3 is T-shaped, SPY-4 is square-planar and T-4_SC is distorted face-capped tetrahedron (Hartshorn et al., 2007).

set due to unreliable localization of the $\mathrm{H}$-atom position in the hydrogen sulfate anion. The distribution of the sulfate-anion connectivity demonstrates (see Table S3 in the supporting information) that the $\mathrm{K}^{4}$ connection type found in (I), (II) and (IV) is not common and accounts for only $1.26 \%$ (13 examples) among 1034 sulfate anions found in the set of crystal structures without disorder of the sulfate anion. The $\mathrm{K}^{21}$ connection type found in (III) is even rarer, with only one example. For sulfate anions that are hydrogen bonded to four neighbouring molecules, the frequency of occurrence of connection types goes down according to $\mathrm{K}^{04}>\mathrm{K}^{13}>\mathrm{K}^{22}>\mathrm{T}^{4}$ $>\mathrm{K}^{4}>\mathrm{K}^{31}$ (see Fig. $\mathrm{S} 1$ in the supporting information), i.e. typically, the sulfate anion forms two hydrogen bonds via each $\mathrm{O}$ atom $\left(\mathrm{K}^{04}\right)$. The other feature to be examined was the coordination modes of the molecular ions in (I)-(IV). The data on the coordination figures of the ions are presented in Fig. 7. Additionally, the coordination figure similarities were calculated for complex cations in the set of 20 structures and sulfate anions in the large set of 834 structures described above. Only six structures out of 20 contain the complex cation with MCTS $\mathrm{K}^{4}$, i.e. hydrogen bonded to four sulfate $\mathrm{O}$ atoms as in (I) and (II) (see Table S2 in the supporting information). The see-saw coordination SS-4 shown in Fig. 7 (which can be seen as a distorted tetrahedron) is typical for this cation, the same coordination figure that was realized in structures (I) and (II), though there are two examples of the tetrahedral T-4 coordination mode. In structure (III), five $\mathrm{O}$ atoms (four from sulfate and one from water) result in the T-4_SC (distorted face-capped tetrahedron) coordination mode found in $\left[M\left(\mathrm{H}_{2} L\right)_{2}\right]^{2+}$ picrates. The distribution of the coordination figures determined for 1020 (14 sulfate anions form only one hydrogen bond, i.e. the ion is monocoordinated) independent sulfate anions from the large set of sulfate structures (see Table S4 in the supporting information) reveals that for 72 sulfate anions coordinated by four $\mathrm{H}$ atoms only: the SPY-4 (noncoplanar, 36 examples) and SS-4 (see-saw, 31 examples) coordination modes prevail, with only five structures featuring tetrahedral T-4 coordination. That means that in (I), (II) and (IV), all the common coordination modes of the sulfate anion were manifested. In structure (III), the TS-3 (T-shaped) coordination mode was found; this appeared in 12 out of 31 structures with a sulfate anion bonded to three $\mathrm{H}$ atoms.

\subsection{Theoretical hydrogen-bonded networks}

In order to explore the probable network topologies of the crystal structures of (I), (II) and (IV) with four-coordinated underlying nets, a set of similar hydrogen-bonded crystal
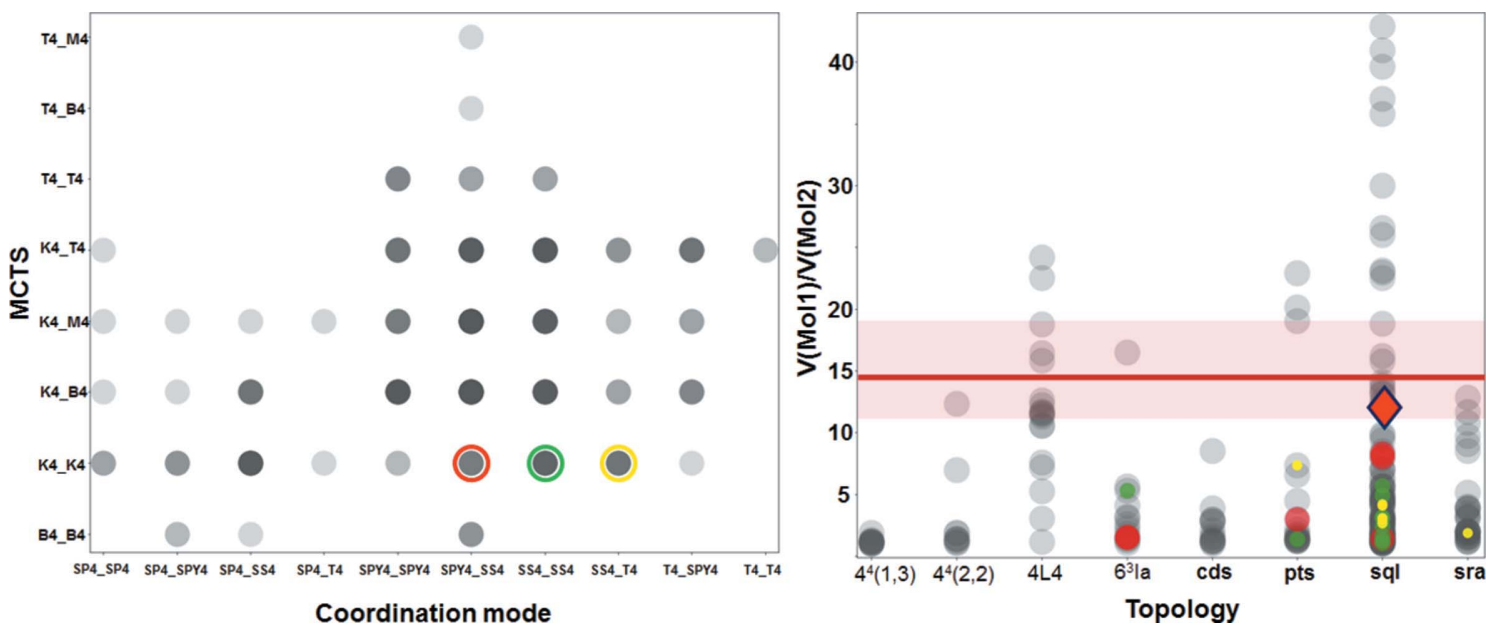

Figure 8

(Left) Two-dimensional frequency plot of MCTS versus coordination mode of molecules in the set of 239 structures defined in $\$ 3.3$. Red, green and yellow circles define the pairs of MCTS-coordination mode found in structures (IV), (II) and (I), respectively. The shade of a point depends on the number of structures with a given combination of MCTS and coordination mode (more transparent points correspond to pairs with smaller numbers of representatives). (Right) Two-dimensional frequency plot of the molecular volume ratio versus hydrogen-bond network topology. Coloured points correspond to the structures with pairs of MCTS-coordination mode observed in (I), (II) and (IV). The red line and pale-red rectangle correspond, respectively, to the $V_{\text {mean }}\left[M\left(\mathrm{H}_{2} L\right)_{2}\right] / V_{\text {mean }}\left(\mathrm{SO}_{4}\right)=14.4$ and to the span of the same ratio, taking into account standard deviations of molecular volumes. The red diamond corresponds to the structure of (IV). Data analysis was performed by means of the Python glue library (Goodman, 2012). 
structures composed of two crystallographically non-equivalent molecules (one molecule comprises only hydrogen-bond donors and the other only hydrogen-bond acceptors) was extracted from the CSD by means of the ToposPro program (Blatov et al., 2014). The connection types of molecules were of the $\mathrm{L}^{4}(\mathrm{~L}=\mathrm{K}, \mathrm{T}, \mathrm{B}$ and $\mathrm{M})$ type, with four single hydrogen bonds and more than 10 representatives for each topological type. As a result, 239 (see Table S5 in the supporting information) structures were retrieved. To take into account the relative dimensions of the molecules, the ratio of molecular volumes (calculated using molecular Voronoi polyhedra) was added to the list of structural descriptors (MCTS and coordination mode). For structures (I), (II) and (IV), the values are 16.0, 16.1 and 12.0, respectively. The mean molecular volumes and their standard deviations (SD) are $V_{\text {mean }}\left[M\left(\mathrm{H}_{2} L\right)_{2}\right]=775 \AA^{3}(\mathrm{SD}=42.8)$ and $V_{\text {mean }}\left(\mathrm{SO}_{4}\right)=$ $53.7 \AA^{3}$ (SD = 11.3), computed from the volumes of 13 complex cations and 1034 sulfate anions. Now, knowing the typical connectivities and coordination modes of both complex cation and sulfate anion, we can conclude that the sql topology observed for unsolvated (IV) (SS-4/SPY-4) was expected to appear because structures with the same molecular connectivity $\mathrm{K}^{4}$ and a molecular volume ratio closer to $V_{\text {mean }}\left[\mathrm{Me}\left(\mathrm{H}_{2} L\right)_{2}\right] / V_{\text {mean }}\left(\mathrm{SO}_{4}\right)=775 / 53.7=14.4$, also have an sql topology [8 (MUSHUD; Plass \& Yozgatli, 2003) and 8.3 (WUCKUC; Moon \& Choi, 2015)] (Fig. 8). Besides, one can see that other topological types for a given coordination mode were realized with a smaller $V(\operatorname{Mol} 1) / V(\operatorname{Mol} 2)$ ratio, i.e. where the two molecules have comparable volumes, which is not the case for the sulfate anion and the $\left[\mathrm{Ni}\left(\mathrm{H}_{2} L\right)_{2}\right]^{2+}$ cation. This, again, emphasizes the significance of the solvent molecules that might direct the crystallization of porous structures (I) and (II) and lead to such unusual topologies.

\section{Final remarks}

The $\left[\mathrm{Ni}\left(\mathrm{H}_{2} L\right)_{2}\right]^{2+}\left(\mathrm{SO}_{4}\right)^{2-}$ system, despite having simple building blocks with only four active centres each, shows the importance of the solvent, which can unpredictably change the outcome of the crystallization process. The unsolvated structure (IV) was probably to be expected based on the analysis of the set of related structures, but structures (I) and (II), evidently, are the outcome of the structure-directing influence of solvents. Structure (III) can be seen as an intermediate step en route to unsolvated polymorph (IV) due to very similar tubular hydrogen-bonded fragments. Even if we are on our way towards the further screening of this promising system through the diversification of solvents, metals and linkers, the challenge posed is extremely difficult to tackle, due to the unpredictability of the synthetic outcomes, a fact supported by the topological and structural analysis of the sets of similar structures. Our present efforts are focused on minimizing these latter drawbacks.

\section{Acknowledgements}

SS thanks CONICET for a research fellowship.

\section{Funding information}

Funding for this research was provided by: Agencia Nacional de Promoción Científica y Tecnológica (project No. PME 2006-01113, for the purchase of the Oxford Gemini CCD diffractometer).

\section{References}

Alexandrov, E. V., Blatov, V. A., Kochetkov, A. V. \& Proserpio, D. M. (2011). CrystEngComm, 11, 3947-3958.

Aman, F., Asiri, A. M., Siddiqui, W. A., Arshad, M. N., Ashraf, A., Zakharov, N. S. \& Blatov, V. A. (2014). CrystEngComm, 16, $1963-$ 1970.

Blatov, V. A. (2016). Struct. Chem. 27, 1605-1611.

Blatov, V. A., Shevchenko, A. P. \& Proserpio, D. M. (2014). Cryst. Growth Des. 14, 3576-3586.

Bruno, I. J., Cole, J. C., Edgington, P. R., Kessler, M., Macrae, C. F., McCabe, P., Pearson, J. \& Taylor, R. (2002). Acta Cryst. B58, 389397.

Goodman, A. A. (2012). Astron. Nachr. 333, 505-514.

Groom, C. R., Bruno, I. J., Lightfoot, M. P. \& Ward, S. C. (2016). Acta Cryst. B72, 171-179.

Guo, Y. C., Chen, S. Y., Qiu, D. F., Feng, Y. Q. \& Song, W. H. (2011). Chin. J. Inorg. Chem. 27, 1517-1520.

Hartshorn, R. M., Hey-Hawkins, E., Kalio, R. \& Leigh, G. J. (2007). Pure Appl. Chem. 79, 1779-1799.

Harvey, M. A., Baggio, S., Ibañez, A. \& Baggio, R. (2004). Acta Cryst. C60, m375-m381.

Harvey, M. A., Baggio, S., Muñoz, J. C. \& Baggio, R. (2003). Acta Cryst. C59, m283-m285.

Harvey, M. A., Suarez, S., Doctorovich, F. \& Baggio, R. (2013). Acta Cryst. C69, 47-51.

Koch, E. \& Fischer, W. (1978). Z. Kristallogr. 148, 107-152.

Larsson, K. \& Öhrström, L. (2003). CrystEngComm, 5, 222-225.

Moon, D. \& Choi, J.-H. (2015). Acta Cryst. E71, 210-212.

O'Keeffe, M., Peskov, M. A., Ramsden, S. J. \& Yaghi, O. M. (2008). Acc. Chem. Res. 41, 1782-1789.

Oxford Diffraction (2009). CrysAlis PRO. Oxford Diffraction Ltd, Yarnton, Oxfordshire, England.

Parsons, S., Flack, H. D. \& Wagner, T. (2013). Acta Cryst. B69, 249 259.

Plass, W. \& Yozgatli, H.-P. (2003). Z. Anorg. Allg. Chem. 629, 65-70.

Sheldrick, G. M. (1996). SADABS. University of Göttingen, Germany.

Sheldrick, G. M. (2008). Acta Cryst. A64, 112-122.

Sheldrick, G. M. (2015). Acta Cryst. C71, 3-8.

Shevchenko, A. P., Blatov, I. A., Kitaevaand, E. V. \& Blatov, V. A. (2017). Cryst. Growth Des. 17, 774-785.

Spek, A. L. (2009). Acta Cryst. D65, 148-155.

Spek, A. L. (2015). Acta Cryst. C71, 9-18.

Zolotarev, P. N., Arshad, M. N., Asiri, A. M., Al-Amshany, Z. M. \& Blatov, V. A. (2014). Cryst. Growth Des. 14, 1938-1949. 


\section{supporting information}

Acta Cryst. (2018). C74, 351-359 [https://doi.org/10.1107/S2053229618002413]

\section{Topological study of diverse hydrogen-bonded patterns found in a system of a nickel(II) complex and the sulfate anion}

\section{Miguel Angel Harvey, Sebastián Suarez, Pavel N. Zolotarev, Davide M. Proserpio and Ricardo Baggio}

Computing details

For all structures, data collection: CrysAlis PRO (Oxford Diffraction, 2009); cell refinement: CrysAlis PRO (Oxford Diffraction, 2009); data reduction: CrysAlis PRO (Oxford Diffraction, 2009); program(s) used to solve structure:

SHELXS97 (Sheldrick, 2008); program(s) used to refine structure: SHELXL2016 (Sheldrick, 2015); molecular graphics: SHELXTL (Sheldrick, 2008); software used to prepare material for publication: SHELXL2014 (Sheldrick, 2015) and PLATON (Spek, 2009).

Bis[2,6-bis(1H-benzimidazol-2-yl- $\left.\kappa N^{3}\right)$ pyridine- $\left.\kappa N\right]$ nickel(II) sulfate (I)

Crystal data

$\left[\mathrm{Ni}\left(\mathrm{C}_{19} \mathrm{H}_{13} \mathrm{~N}_{5}\right)_{2}\right] \mathrm{SO}_{4}$

$M_{r}=777.46$

Orthorhombic, $P 22_{1} 2_{1} 2_{1}$

$a=9.8015(6) \AA$

$b=20.9572(15) \AA$

$c=21.7012(17) \AA$

$V=4457.7(5) \AA^{3}$

$Z=4$

$F(000)=1600$

\section{Data collection}

Oxford Diffraction Gemini CCD S Ultra diffractometer

Radiation source: fine-focus sealed tube Graphite monochromator $\omega$ scans, thick slices

Absorption correction: multi-scan (CrysAlis PRO; Oxford Diffraction, 2009)

$T_{\min }=0.82, T_{\max }=0.92$

Refinement

Refinement on $F^{2}$ Least-squares matrix: full $R\left[F^{2}>2 \sigma\left(F^{2}\right)\right]=0.074$

$w R\left(F^{2}\right)=0.210$

$S=0.98$

9840 reflections

487 parameters
$D_{\mathrm{x}}=1.158 \mathrm{Mg} \mathrm{m}^{-3}$

Mo $K \alpha$ radiation, $\lambda=0.71073 \AA$

Cell parameters from 4887 reflections

$\theta=3.8-28.1^{\circ}$

$\mu=0.53 \mathrm{~mm}^{-1}$

$T=294 \mathrm{~K}$

Needle, light_red

$0.50 \times 0.24 \times 0.18 \mathrm{~mm}$

19326 measured reflections

9840 independent reflections

5651 reflections with $I>2 \sigma(I)$

$R_{\text {int }}=0.085$

$\theta_{\max }=28.9^{\circ}, \theta_{\min }=3.6^{\circ}$

$h=-12 \rightarrow 11$

$k=-26 \rightarrow 28$

$l=-26 \rightarrow 28$

586 restraints

Hydrogen site location: inferred from neighbouring sites

$\mathrm{H}$-atom parameters constrained

$w=1 /\left[\sigma^{2}\left(F_{\mathrm{o}}^{2}\right)+(0.0908 P)^{2}\right]$

where $P=\left(F_{\mathrm{o}}^{2}+2 F_{\mathrm{c}}^{2}\right) / 3$

$(\Delta / \sigma)_{\max }<0.001$ 


$$
\Delta \rho_{\max }=0.47 \mathrm{e} \AA^{-3}
$$$$
\Delta \rho_{\min }=-0.31 \text { e } \AA^{-3}
$$

Absolute structure: Flack $x$ determined using 1523 quotients $[(\mathrm{I}+)-(\mathrm{I}-)] /[(\mathrm{I}+)+(\mathrm{I}-)]$ (Parsons et al., 2013)

Absolute structure parameter: 0.006 (19)

Special details

Geometry. All e.s.d.'s (except the e.s.d. in the dihedral angle between two 1.s. planes) are estimated using the full covariance matrix. The cell e.s.d.'s are taken into account individually in the estimation of e.s.d.'s in distances, angles and torsion angles; correlations between e.s.d.'s in cell parameters are only used when they are defined by crystal symmetry. An approximate (isotropic) treatment of cell e.s.d.'s is used for estimating e.s.d.'s involving l.s. planes.

Fractional atomic coordinates and isotropic or equivalent isotropic displacement parameters $\left(\hat{A}^{2}\right)$

\begin{tabular}{|c|c|c|c|c|}
\hline & $x$ & $y$ & $z$ & $U_{\text {iso }} * / U_{\text {eq }}$ \\
\hline Ni1 & $0.88293(9)$ & $0.08567(5)$ & $0.84243(4)$ & $0.0392(3)$ \\
\hline N1A & $0.6872(5)$ & $0.0444(3)$ & $0.8476(3)$ & $0.0448(15)$ \\
\hline $\mathrm{N} 2 \mathrm{~A}$ & $0.8989(5)$ & 0.0117 & 0.7826 & $0.0424(14)$ \\
\hline N3A & $1.0826(5)$ & $0.0964(3)$ & $0.8072(3)$ & 0.0439 (14) \\
\hline N4A & $0.5513(6)$ & -0.0333 & $0.8142(3)$ & $0.0555(18)$ \\
\hline H4NA & 0.523803 & -0.066115 & 0.793923 & $0.067 *$ \\
\hline N5A & $1.2442(6)$ & $0.0573(3)$ & 0.7459 & $0.0508(17)$ \\
\hline H5NA & 1.285383 & 0.031955 & 0.720939 & $0.061 *$ \\
\hline $\mathrm{C} 1 \mathrm{~A}$ & $0.5640(7)$ & $0.0489(4)$ & $0.8785(4)$ & 0.0469 (18) \\
\hline $\mathrm{C} 2 \mathrm{~A}$ & $0.5188(8)$ & $0.0932(4)$ & $0.9213(4)$ & $0.061(2)$ \\
\hline $\mathrm{H} 2 \mathrm{AA}$ & 0.572642 & 0.126997 & 0.934752 & $0.074^{*}$ \\
\hline $\mathrm{C} 3 \mathrm{~A}$ & $0.3880(8)$ & $0.0832(5)$ & $0.9424(5)$ & $0.080(3)$ \\
\hline H3AA & 0.353593 & 0.111188 & 0.971828 & $0.096^{*}$ \\
\hline $\mathrm{C} 4 \mathrm{~A}$ & $0.3063(9)$ & $0.0350(5)$ & $0.9228(5)$ & $0.075(3)$ \\
\hline H4AA & 0.219539 & 0.030882 & 0.939718 & $0.090 *$ \\
\hline C5A & $0.3475(7)$ & $-0.0071(5)$ & 0.8795 & $0.066(2)$ \\
\hline H5AA & 0.290457 & -0.039530 & 0.865700 & $0.079 *$ \\
\hline C6A & $0.4781(7)$ & $0.0003(4)$ & $0.8567(4)$ & $0.0482(18)$ \\
\hline C7A & $0.6749(7)$ & $-0.0053(3)$ & $0.8099(3)$ & 0.0461 (19) \\
\hline C8A & $0.7926(7)$ & -0.0270 & $0.7752(4)$ & $0.052(2)$ \\
\hline C9A & $0.8036(8)$ & $-0.0816(5)$ & $0.7394(5)$ & $0.076(3)$ \\
\hline H9AA & 0.728977 & -0.108296 & 0.733392 & $0.091 *$ \\
\hline C10A & $0.9262(9)$ & $-0.0949(5)$ & $0.7132(5)$ & $0.090(4)$ \\
\hline $\mathrm{H} 10 \mathrm{~A}$ & 0.935425 & -0.131541 & 0.689406 & $0.108^{*}$ \\
\hline C11A & $1.0384(9)$ & $-0.0550(4)$ & $0.7212(5)$ & $0.072(3)$ \\
\hline H11A & 1.122472 & -0.064533 & 0.703554 & $0.086^{*}$ \\
\hline $\mathrm{C} 12 \mathrm{~A}$ & $1.0199(6)$ & $-0.0005(3)$ & $0.7564(4)$ & $0.0457(18)$ \\
\hline C13A & $1.1186(7)$ & $0.0496(3)$ & $0.7702(3)$ & 0.0435 (16) \\
\hline C14A & $1.2933(7)$ & $0.1143(3)$ & $0.7692(4)$ & $0.0482(18)$ \\
\hline $\mathrm{C} 15 \mathrm{~A}$ & $1.4155(8)$ & $0.1466(4)$ & $0.7584(5)$ & $0.067(3)$ \\
\hline $\mathrm{H} 15 \mathrm{~A}$ & 1.483524 & 0.129796 & 0.733345 & $0.081 *$ \\
\hline C16A & $1.4288(9)$ & $0.2035(5)$ & $0.7866(5)$ & $0.076(3)$ \\
\hline H16A & 1.508808 & 0.226567 & 0.780600 & $0.092 *$ \\
\hline C17A & $1.3282(8)$ & 0.2293 & $0.8244(5)$ & $0.071(3)$ \\
\hline H17A & 1.342304 & 0.269074 & 0.842373 & $0.086^{*}$ \\
\hline
\end{tabular}




\begin{tabular}{|c|c|c|c|c|}
\hline C18A & $1.2091(8)$ & 0.1975 (4) & $0.8356(5)$ & $0.061(2)$ \\
\hline H18A & 1.141536 & 0.214640 & 0.860656 & $0.073^{*}$ \\
\hline C19A & $1.1936(7)$ & 0.1381 (3) & 0.8076 (4) & 0.0469 (18) \\
\hline N1B & $0.8202(6)$ & 0.1655 (3) & 0.7902 (3) & $0.0430(15)$ \\
\hline $\mathrm{N} 2 \mathrm{~B}$ & $0.8719(6)$ & $0.1553(2)$ & 0.9068 (2) & $0.0371(13)$ \\
\hline N3B & $0.9392(6)$ & $0.0370(2)$ & $0.9242(3)$ & $0.0411(14)$ \\
\hline N4B & $0.7729(6)$ & $0.2694(3)$ & $0.7912(3)$ & 0.0458 \\
\hline H4NB & 0.760629 & 0.307484 & 0.804941 & $0.055^{*}$ \\
\hline N5B & $0.9636(7)$ & $0.0440(3)$ & $1.0261(3)$ & $0.0473(15)$ \\
\hline H5NB & 0.965944 & 0.060504 & 1.062391 & $0.057^{*}$ \\
\hline C1B & $0.7895(7)$ & $0.1843(3)$ & $0.7306(3)$ & $0.0454(17)$ \\
\hline $\mathrm{C} 2 \mathrm{~B}$ & $0.7839(10)$ & 0.1499 (4) & 0.6764 (3) & $0.065(2)$ \\
\hline $\mathrm{H} 2 \mathrm{BA}$ & 0.804359 & 0.106636 & 0.674822 & $0.078^{*}$ \\
\hline C3B & $0.7462(11)$ & $0.1837(5)$ & 0.6247 (4) & $0.075(3)$ \\
\hline H3BA & 0.740590 & 0.162069 & 0.587424 & $0.090 *$ \\
\hline C4B & $0.7168(11)$ & $0.2469(5)$ & $0.6253(4)$ & 0.075 \\
\hline H4BA & 0.690994 & 0.266911 & 0.588882 & $0.090^{*}$ \\
\hline C5B & $0.7242(10)$ & 0.2814 (4) & 0.6777 (4) & $0.067(3)$ \\
\hline H5BA & 0.704945 & 0.324872 & 0.677771 & $0.080 *$ \\
\hline C6B & $0.7615(8)$ & $0.2499(3)$ & $0.7314(3)$ & $0.0460(17)$ \\
\hline C7B & $0.8070(8)$ & $0.2178(3)$ & $0.8246(3)$ & $0.0411(16)$ \\
\hline $\mathrm{C} 8 \mathrm{~B}$ & $0.8339(9)$ & $0.2138(3)$ & $0.8902(3)$ & 0.0477 (19) \\
\hline C9B & $0.8197(15)$ & 0.2618 (4) & 0.9337 (4) & 0.099 (4) \\
\hline H9BA & 0.792888 & 0.302607 & 0.922180 & $0.118^{*}$ \\
\hline $\mathrm{C} 10 \mathrm{~B}$ & $0.8460(17)$ & $0.2477(5)$ & $0.9936(4)$ & $0.123(6)$ \\
\hline H10B & 0.831651 & 0.278571 & 1.023653 & $0.148^{*}$ \\
\hline C11B & $0.8940(15)$ & 0.1877 (4) & $1.0108(4)$ & $0.099(4)$ \\
\hline H11B & 0.920952 & 0.179170 & 1.051076 & $0.119 *$ \\
\hline C12B & $0.8998(9)$ & $0.1412(3)$ & 0.9654 (3) & $0.050(2)$ \\
\hline C13B & $0.9343(7)$ & $0.0740(3)$ & $0.9728(3)$ & $0.0408(15)$ \\
\hline C14B & $0.9891(8)$ & $-0.0188(3)$ & $1.0106(3)$ & $0.0447(17)$ \\
\hline C15B & $1.0240(9)$ & $-0.0708(3)$ & 1.0469 (4) & $0.064(2)$ \\
\hline H15B & 1.034589 & -0.067328 & 1.089340 & $0.077 *$ \\
\hline C16B & $1.0417(11)$ & $-0.1266(4)$ & $1.0173(5)$ & $0.079(3)$ \\
\hline H16B & 1.063909 & -0.162706 & 1.040103 & $0.095 *$ \\
\hline C17B & $1.0280(11)$ & $-0.1322(4)$ & $0.9537(5)$ & $0.077(3)$ \\
\hline H17B & 1.043347 & -0.171601 & 0.935233 & $0.093 *$ \\
\hline C18B & $0.9925(9)$ & -0.0814 & 0.9179 (4) & $0.062(2)$ \\
\hline H18B & 0.980261 & -0.085581 & 0.875547 & $0.074 *$ \\
\hline C19B & $0.9754(8)$ & $-0.0230(3)$ & $0.9475(3)$ & $0.0464(18)$ \\
\hline $\mathrm{S} 1$ & $1.0813(2)$ & $0.09127(10)$ & $1.17397(10)$ & $0.0506(5)$ \\
\hline O1 & $1.1035(7)$ & $0.0222(3)$ & $1.1762(3)$ & $0.0732(19)$ \\
\hline $\mathrm{O} 2$ & $1.0534(7)$ & 0.1175 (3) & $1.2361(3)$ & $0.0677(18)$ \\
\hline $\mathrm{O} 3$ & $0.9641(6)$ & $0.1041(3)$ & $1.1343(3)$ & 0.0707 (19) \\
\hline O4 & $1.2034(6)$ & $0.1228(3)$ & $1.1497(3)$ & 0.0699 (18) \\
\hline
\end{tabular}


Atomic displacement parameters $\left(\AA^{2}\right)$

\begin{tabular}{|c|c|c|c|c|c|c|}
\hline & $U^{11}$ & $U^{22}$ & $U^{33}$ & $U^{12}$ & $U^{13}$ & $U^{23}$ \\
\hline Nil & $0.0392(5)$ & $0.0372(5)$ & $0.0412(5)$ & $-0.0011(5)$ & $0.0003(4)$ & $-0.0053(5)$ \\
\hline N1A & $0.043(3)$ & $0.043(3)$ & $0.048(4)$ & $-0.001(3)$ & $0.002(3)$ & -0.009 \\
\hline N2A & $0.041(3)$ & $0.038(3)$ & $0.048(4)$ & $-0.002(2)$ & $0.005(2)$ & -0.010 \\
\hline N3A & $0.042(3)$ & $0.044(3)$ & $0.046(3)$ & $-0.004(2)$ & $0.004(2)$ & $-0.006(3)$ \\
\hline $\mathrm{N} 4 \mathrm{~A}$ & $0.053(3)$ & $0.050(4)$ & $0.064(4)$ & $-0.016(3)$ & $0.005(3)$ & $-0.012(4)$ \\
\hline N5A & $0.046(3)$ & $0.054(3)$ & $0.053(4)$ & $-0.003(3)$ & $0.014(3)$ & $-0.006(3)$ \\
\hline $\mathrm{C} 1 \mathrm{~A}$ & $0.039(3)$ & $0.047(4)$ & $0.055(4)$ & 0.003 & $0.000(3)$ & $0.000(3)$ \\
\hline $\mathrm{C} 2 \mathrm{~A}$ & $0.053(4)$ & $0.053(4)$ & $0.078(6)$ & $-0.004(4)$ & $0.017(4)$ & $-0.012(4)$ \\
\hline $\mathrm{C} 3 \mathrm{~A}$ & $0.057(4)$ & $0.073(5)$ & $0.109(8)$ & $-0.003(4)$ & $0.029(4)$ & $-0.007(6)$ \\
\hline $\mathrm{C} 4 \mathrm{~A}$ & $0.056(5)$ & $0.076(5)$ & $0.093(7)$ & $-0.007(4)$ & $0.021(5)$ & $0.005(5)$ \\
\hline C5A & $0.045(4)$ & $0.076(6)$ & 0.077 (6) & -0.009 (4) & $0.002(4)$ & $0.005(5)$ \\
\hline C6A & $0.044(3)$ & $0.048(4)$ & $0.053(5)$ & $-0.001(3)$ & $-0.002(3)$ & $0.005(3)$ \\
\hline C7A & $0.046(3)$ & $0.045(4)$ & 0.048 (4) & -0.009 (3) & $0.004(3)$ & -0.008 \\
\hline C8A & 0.049 (3) & $0.051(4)$ & $0.056(5)$ & $-0.013(3)$ & $0.008(3)$ & $-0.016(4)$ \\
\hline C9A & $0.072(5)$ & $0.069(5)$ & $0.088(7)$ & $-0.021(5)$ & $0.015(5)$ & $-0.041(5)$ \\
\hline $\mathrm{C} 10 \mathrm{~A}$ & $0.086(5)$ & $0.066(6)$ & $0.118(9)$ & $-0.016(4)$ & $0.032(5)$ & $-0.053(7)$ \\
\hline C11A & $0.072(5)$ & $0.060(4)$ & $0.083(7)$ & $-0.006(4)$ & $0.025(5)$ & $-0.029(5)$ \\
\hline $\mathrm{C} 12 \mathrm{~A}$ & $0.044(3)$ & $0.046(3)$ & $0.047(5)$ & -0.001 & $0.008(3)$ & $-0.008(3)$ \\
\hline C13A & $0.042(3)$ & $0.044(3)$ & $0.044(4)$ & $-0.001(3)$ & $0.008(3)$ & $-0.003(3)$ \\
\hline C14A & $0.046(3)$ & $0.050(3)$ & $0.049(5)$ & $-0.003(3)$ & $-0.001(3)$ & $0.006(3)$ \\
\hline C15A & $0.055(4)$ & $0.074(5)$ & $0.073(6)$ & $-0.017(4)$ & $0.008(4)$ & $0.004(5)$ \\
\hline C16A & $0.058(5)$ & $0.083(5)$ & $0.088(7)$ & $-0.027(4)$ & $0.005(4)$ & $-0.006(5)$ \\
\hline C17A & $0.066(4)$ & $0.069(6)$ & $0.079(7)$ & $-0.024(4)$ & $0.001(4)$ & $-0.008(5)$ \\
\hline C18A & $0.058(4)$ & $0.056(4)$ & $0.068(6)$ & $-0.013(3)$ & $0.004(4)$ & $-0.011(4)$ \\
\hline C19A & $0.045(3)$ & $0.048(3)$ & $0.048(4)$ & -0.007 & $-0.002(3)$ & $0.002(3)$ \\
\hline N1B & 0.049 (4) & $0.039(3)$ & $0.041(3)$ & -0.001 & -0.003 & $-0.004(2)$ \\
\hline N2B & $0.038(3)$ & $0.035(2)$ & $0.038(3)$ & $0.001(3)$ & $-0.002(3)$ & $0.000(2)$ \\
\hline N3B & $0.040(3)$ & $0.035(2)$ & $0.048(3)$ & $0.001(3)$ & $0.000(3)$ & $-0.004(2)$ \\
\hline N4B & $0.048(4)$ & $0.046(3)$ & $0.044(3)$ & $0.006(3)$ & -0.003 & 0.007 (3) \\
\hline N5B & $0.053(4)$ & $0.040(3)$ & $0.049(3)$ & -0.001 & $-0.006(3)$ & $0.000(3)$ \\
\hline C1B & $0.040(4)$ & $0.055(3)$ & $0.041(3)$ & -0.001 & $-0.001(3)$ & $0.002(3)$ \\
\hline $\mathrm{C} 2 \mathrm{~B}$ & $0.081(6)$ & $0.068(5)$ & $0.045(3)$ & $0.011(5)$ & -0.005 & $-0.006(3)$ \\
\hline C3B & $0.094(7)$ & $0.092(5)$ & $0.039(4)$ & $0.011(5)$ & $-0.011(5)$ & $-0.004(4)$ \\
\hline C4B & $0.087(7)$ & $0.093(5)$ & $0.045(4)$ & $0.013(5)$ & $0.002(5)$ & $0.010(4)$ \\
\hline C5B & $0.080(6)$ & $0.076(6)$ & $0.044(3)$ & $0.018(5)$ & $0.003(4)$ & $0.017(4)$ \\
\hline C6B & $0.042(4)$ & $0.056(3)$ & $0.040(3)$ & $0.003(3)$ & $0.005(3)$ & $0.009(3)$ \\
\hline C7B & $0.048(4)$ & $0.036(3)$ & $0.039(3)$ & $0.002(3)$ & $-0.003(3)$ & $0.000(2)$ \\
\hline $\mathrm{C} 8 \mathrm{~B}$ & $0.069(5)$ & $0.034(3)$ & $0.040(3)$ & $0.006(3)$ & -0.007 & $0.000(3)$ \\
\hline C9B & $0.198(13)$ & $0.047(5)$ & $0.051(4)$ & $0.037(7)$ & $-0.033(6)$ & $-0.012(4)$ \\
\hline C10B & $0.261(17)$ & $0.057(5)$ & $0.051(4)$ & $0.051(8)$ & $-0.040(7)$ & $-0.017(5)$ \\
\hline C11B & $0.198(12)$ & $0.053(4)$ & $0.045(5)$ & $0.032(6)$ & $-0.033(7)$ & $-0.012(4)$ \\
\hline C12B & $0.072(5)$ & $0.041(3)$ & 0.039 (3) & 0.008 (4) & -0.008 & $-0.002(3)$ \\
\hline C13B & $0.040(4)$ & $0.037(3)$ & $0.046(3)$ & $0.000(3)$ & $-0.004(3)$ & $-0.004(2)$ \\
\hline C14B & $0.042(4)$ & $0.038(3)$ & $0.055(3)$ & $-0.003(3)$ & -0.003 & $0.004(3)$ \\
\hline C15B & $0.076(6)$ & 0.047 (4) & $0.068(5)$ & $0.007(4)$ & $-0.001(5)$ & $0.015(3)$ \\
\hline
\end{tabular}




\begin{tabular}{lllllll} 
C16B & $0.096(7)$ & $0.047(4)$ & $0.096(5)$ & $0.015(5)$ & $0.002(6)$ & $0.010(5)$ \\
C17B & $0.095(7)$ & $0.039(4)$ & $0.098(5)$ & $0.009(5)$ & $0.001(6)$ & $0.000(4)$ \\
C18B & $0.070(5)$ & $0.040(3)$ & $0.075(5)$ & $0.001(4)$ & $-0.004(4)$ & $-0.010(3)$ \\
C19B & $0.047(4)$ & $0.036(3)$ & $0.056(4)$ & $-0.001(3)$ & $-0.005(3)$ & $-0.002(3)$ \\
S1 & $0.0652(13)$ & $0.0423(10)$ & $0.0444(11)$ & $-0.0060(11)$ & $-0.0112(9)$ & $0.0104(11)$ \\
O1 & $0.086(4)$ & $0.039(3)$ & $0.094(5)$ & $-0.008(3)$ & $-0.038(4)$ & $0.012(3)$ \\
O2 & $0.096(4)$ & $0.070(4)$ & $0.037(3)$ & $-0.033(4)$ & $0.001(3)$ & $0.004(3)$ \\
O3 & $0.072(4)$ & $0.083(5)$ & $0.057(4)$ & $0.014(4)$ & $-0.025(3)$ & $0.002(4)$ \\
O4 & $0.076(4)$ & $0.055(3)$ & $0.079(5)$ & $-0.019(3)$ & $0.015(4)$ & $0.003(4)$ \\
\hline
\end{tabular}

Geometric parameters $\left(A,{ }^{\circ}\right)$

\begin{tabular}{|c|c|c|c|}
\hline Ni1-N2B & $2.022(5)$ & $\mathrm{C} 18 \mathrm{~A}-\mathrm{H} 18 \mathrm{~A}$ & 0.9300 \\
\hline $\mathrm{Ni} 1-\mathrm{N} 2 \mathrm{~A}$ & $2.029(5)$ & $\mathrm{N} 1 \mathrm{~B}-\mathrm{C} 7 \mathrm{~B}$ & $1.333(7)$ \\
\hline Ni1-N1A & $2.108(5)$ & $\mathrm{N} 1 \mathrm{~B}-\mathrm{C} 1 \mathrm{~B}$ & $1.384(8)$ \\
\hline Ni1-N1B & $2.112(6)$ & $\mathrm{N} 2 \mathrm{~B}-\mathrm{C} 8 \mathrm{~B}$ & $1.330(7)$ \\
\hline $\mathrm{Ni} 1-\mathrm{N} 3 \mathrm{~A}$ & $2.113(5)$ & $\mathrm{N} 2 \mathrm{~B}-\mathrm{C} 12 \mathrm{~B}$ & $1.335(7)$ \\
\hline Ni1-N3B & $2.120(6)$ & $\mathrm{N} 3 \mathrm{~B}-\mathrm{C} 13 \mathrm{~B}$ & $1.311(7)$ \\
\hline $\mathrm{N} 1 \mathrm{~A}-\mathrm{C} 7 \mathrm{~A}$ & $1.328(7)$ & $\mathrm{N} 3 \mathrm{~B}-\mathrm{C} 19 \mathrm{~B}$ & $1.400(7)$ \\
\hline $\mathrm{N} 1 \mathrm{~A}-\mathrm{C} 1 \mathrm{~A}$ & $1.385(8)$ & $\mathrm{N} 4 \mathrm{~B}-\mathrm{C} 7 \mathrm{~B}$ & $1.345(7)$ \\
\hline $\mathrm{N} 2 \mathrm{~A}-\mathrm{C} 8 \mathrm{~A}$ & $1.330(7)$ & $\mathrm{N} 4 \mathrm{~B}-\mathrm{C} 6 \mathrm{~B}$ & $1.366(8)$ \\
\hline $\mathrm{N} 2 \mathrm{~A}-\mathrm{C} 12 \mathrm{~A}$ & $1.339(7)$ & $\mathrm{N} 4 \mathrm{~B}-\mathrm{H} 4 \mathrm{NB}$ & 0.8600 \\
\hline $\mathrm{N} 3 \mathrm{~A}-\mathrm{C} 13 \mathrm{~A}$ & $1.315(7)$ & $\mathrm{N} 5 \mathrm{~B}-\mathrm{C} 13 \mathrm{~B}$ & $1.350(7)$ \\
\hline $\mathrm{N} 3 \mathrm{~A}-\mathrm{C} 19 \mathrm{~A}$ & $1.396(7)$ & $\mathrm{N} 5 \mathrm{~B}-\mathrm{C} 14 \mathrm{~B}$ & $1.381(7)$ \\
\hline $\mathrm{N} 4 \mathrm{~A}-\mathrm{C} 7 \mathrm{~A}$ & $1.349(7)$ & $\mathrm{N} 5 \mathrm{~B}-\mathrm{H} 5 \mathrm{NB}$ & 0.8600 \\
\hline $\mathrm{N} 4 \mathrm{~A}-\mathrm{C} 6 \mathrm{~A}$ & $1.364(8)$ & $\mathrm{C} 1 \mathrm{~B}-\mathrm{C} 2 \mathrm{~B}$ & $1.381(9)$ \\
\hline $\mathrm{N} 4 \mathrm{~A}-\mathrm{H} 4 \mathrm{NA}$ & 0.8600 & $\mathrm{C} 1 \mathrm{~B}-\mathrm{C} 6 \mathrm{~B}$ & $1.403(9)$ \\
\hline $\mathrm{N} 5 \mathrm{~A}-\mathrm{C} 13 \mathrm{~A}$ & $1.349(7)$ & $\mathrm{C} 2 \mathrm{~B}-\mathrm{C} 3 \mathrm{~B}$ & $1.377(9)$ \\
\hline $\mathrm{N} 5 \mathrm{~A}-\mathrm{C} 14 \mathrm{~A}$ & $1.382(7)$ & $\mathrm{C} 2 \mathrm{~B}-\mathrm{H} 2 \mathrm{BA}$ & 0.9300 \\
\hline N5A-H5NA & 0.8600 & $\mathrm{C} 3 \mathrm{~B}-\mathrm{C} 4 \mathrm{~B}$ & $1.355(11)$ \\
\hline $\mathrm{C} 1 \mathrm{~A}-\mathrm{C} 2 \mathrm{~A}$ & $1.385(9)$ & $\mathrm{C} 3 \mathrm{~B}-\mathrm{H} 3 \mathrm{BA}$ & 0.9300 \\
\hline $\mathrm{C} 1 \mathrm{~A}-\mathrm{C} 6 \mathrm{~A}$ & $1.403(9)$ & $\mathrm{C} 4 \mathrm{~B}-\mathrm{C} 5 \mathrm{~B}$ & $1.348(11)$ \\
\hline $\mathrm{C} 2 \mathrm{~A}-\mathrm{C} 3 \mathrm{~A}$ & $1.378(9)$ & $\mathrm{C} 4 \mathrm{~B}-\mathrm{H} 4 \mathrm{BA}$ & 0.9300 \\
\hline $\mathrm{C} 2 \mathrm{~A}-\mathrm{H} 2 \mathrm{AA}$ & 0.9300 & $\mathrm{C} 5 \mathrm{~B}-\mathrm{C} 6 \mathrm{~B}$ & $1.389(9)$ \\
\hline $\mathrm{C} 3 \mathrm{~A}-\mathrm{C} 4 \mathrm{~A}$ & $1.357(11)$ & $\mathrm{C} 5 \mathrm{~B}-\mathrm{H} 5 \mathrm{BA}$ & 0.9300 \\
\hline $\mathrm{C} 3 \mathrm{~A}-\mathrm{H} 3 \mathrm{AA}$ & 0.9300 & $\mathrm{C} 7 \mathrm{~B}-\mathrm{C} 8 \mathrm{~B}$ & $1.450(9)$ \\
\hline $\mathrm{C} 4 \mathrm{~A}-\mathrm{C} 5 \mathrm{~A}$ & $1.351(10)$ & $\mathrm{C} 8 \mathrm{~B}-\mathrm{C} 9 \mathrm{~B}$ & $1.387(9)$ \\
\hline $\mathrm{C} 4 \mathrm{~A}-\mathrm{H} 4 \mathrm{AA}$ & 0.9300 & $\mathrm{C} 9 \mathrm{~B}-\mathrm{C} 10 \mathrm{~B}$ & $1.357(11)$ \\
\hline $\mathrm{C} 5 \mathrm{~A}-\mathrm{C} 6 \mathrm{~A}$ & $1.382(9)$ & $\mathrm{C} 9 \mathrm{~B}-\mathrm{H} 9 \mathrm{BA}$ & 0.9300 \\
\hline $\mathrm{C} 5 \mathrm{~A}-\mathrm{H} 5 \mathrm{AA}$ & 0.9300 & $\mathrm{C} 10 \mathrm{~B}-\mathrm{C} 11 \mathrm{~B}$ & $1.393(10)$ \\
\hline $\mathrm{C} 7 \mathrm{~A}-\mathrm{C} 8 \mathrm{~A}$ & $1.451(9)$ & $\mathrm{C} 10 \mathrm{~B}-\mathrm{H} 10 \mathrm{~B}$ & 0.9300 \\
\hline $\mathrm{C} 8 \mathrm{~A}-\mathrm{C} 9 \mathrm{~A}$ & $1.388(9)$ & $\mathrm{C} 11 \mathrm{~B}-\mathrm{C} 12 \mathrm{~B}$ & $1.387(9)$ \\
\hline $\mathrm{C} 9 \mathrm{~A}-\mathrm{C} 10 \mathrm{~A}$ & $1.358(10)$ & $\mathrm{C} 11 \mathrm{~B}-\mathrm{H} 11 \mathrm{~B}$ & 0.9300 \\
\hline C9A-H9AA & 0.9300 & $\mathrm{C} 12 \mathrm{~B}-\mathrm{C} 13 \mathrm{~B}$ & $1.456(8)$ \\
\hline $\mathrm{C} 10 \mathrm{~A}-\mathrm{C} 11 \mathrm{~A}$ & $1.393(10)$ & $\mathrm{C} 14 \mathrm{~B}-\mathrm{C} 19 \mathrm{~B}$ & $1.380(9)$ \\
\hline $\mathrm{C} 10 \mathrm{~A}-\mathrm{H} 10 \mathrm{~A}$ & 0.9300 & $\mathrm{C} 14 \mathrm{~B}-\mathrm{C} 15 \mathrm{~B}$ & $1.387(8)$ \\
\hline $\mathrm{C} 11 \mathrm{~A}-\mathrm{C} 12 \mathrm{~A}$ & $1.387(9)$ & $\mathrm{C} 15 \mathrm{~B}-\mathrm{C} 16 \mathrm{~B}$ & $1.345(10)$ \\
\hline $\mathrm{C} 11 \mathrm{~A}-\mathrm{H} 11 \mathrm{~A}$ & 0.9300 & $\mathrm{C} 15 \mathrm{~B}-\mathrm{H} 15 \mathrm{~B}$ & 0.9300 \\
\hline
\end{tabular}




\begin{tabular}{|c|c|c|c|}
\hline $\mathrm{C} 12 \mathrm{~A}-\mathrm{C} 13 \mathrm{~A}$ & $1.458(8)$ & $\mathrm{C} 16 \mathrm{~B}-\mathrm{C} 17 \mathrm{~B}$ & $1.390(11)$ \\
\hline $\mathrm{C} 14 \mathrm{~A}-\mathrm{C} 19 \mathrm{~A}$ & $1.378(9)$ & $\mathrm{C} 16 \mathrm{~B}-\mathrm{H} 16 \mathrm{~B}$ & 0.9300 \\
\hline $\mathrm{C} 14 \mathrm{~A}-\mathrm{C} 15 \mathrm{~A}$ & $1.394(8)$ & $\mathrm{C} 17 \mathrm{~B}-\mathrm{C} 18 \mathrm{~B}$ & $1.364(9)$ \\
\hline $\mathrm{C} 15 \mathrm{~A}-\mathrm{C} 16 \mathrm{~A}$ & $1.348(10)$ & $\mathrm{C} 17 \mathrm{~B}-\mathrm{H} 17 \mathrm{~B}$ & 0.9300 \\
\hline $\mathrm{C} 15 \mathrm{~A}-\mathrm{H} 15 \mathrm{~A}$ & 0.9300 & $\mathrm{C} 18 \mathrm{~B}-\mathrm{C} 19 \mathrm{~B}$ & $1.393(9)$ \\
\hline $\mathrm{C} 16 \mathrm{~A}-\mathrm{C} 17 \mathrm{~A}$ & $1.392(10)$ & $\mathrm{C} 18 \mathrm{~B}-\mathrm{H} 18 \mathrm{~B}$ & 0.9300 \\
\hline $\mathrm{C} 16 \mathrm{~A}-\mathrm{H} 16 \mathrm{~A}$ & 0.9300 & $\mathrm{~S} 1-\mathrm{O} 3$ & $1.461(6)$ \\
\hline $\mathrm{C} 17 \mathrm{~A}-\mathrm{C} 18 \mathrm{~A}$ & $1.367(9)$ & $\mathrm{S} 1-\mathrm{O} 1$ & $1.464(6)$ \\
\hline $\mathrm{C} 17 \mathrm{~A}-\mathrm{H} 17 \mathrm{~A}$ & 0.9300 & $\mathrm{~S} 1-\mathrm{O} 4$ & $1.465(6)$ \\
\hline $\mathrm{C} 18 \mathrm{~A}-\mathrm{C} 19 \mathrm{~A}$ & $1.393(8)$ & $\mathrm{S} 1-\mathrm{O} 2$ & $1.481(6)$ \\
\hline $\mathrm{N} 2 \mathrm{~B}-\mathrm{Ni1}-\mathrm{N} 2 \mathrm{~A}$ & $176.0(2)$ & $\mathrm{C} 19 \mathrm{~A}-\mathrm{C} 18 \mathrm{~A}-\mathrm{H} 18 \mathrm{~A}$ & 121.5 \\
\hline $\mathrm{N} 2 \mathrm{~B}-\mathrm{Ni1}-\mathrm{N} 1 \mathrm{~A}$ & $102.2(2)$ & $\mathrm{C} 14 \mathrm{~A}-\mathrm{C} 19 \mathrm{~A}-\mathrm{C} 18 \mathrm{~A}$ & $120.6(6)$ \\
\hline $\mathrm{N} 2 \mathrm{~A}-\mathrm{Ni1}-\mathrm{N} 1 \mathrm{~A}$ & $77.9(2)$ & $\mathrm{C} 14 \mathrm{~A}-\mathrm{C} 19 \mathrm{~A}-\mathrm{N} 3 \mathrm{~A}$ & $108.8(6)$ \\
\hline $\mathrm{N} 2 \mathrm{~B}-\mathrm{Ni1}-\mathrm{N} 1 \mathrm{~B}$ & $77.5(2)$ & $\mathrm{C} 18 \mathrm{~A}-\mathrm{C} 19 \mathrm{~A}-\mathrm{N} 3 \mathrm{~A}$ & $130.3(6)$ \\
\hline N2A-Ni1-N1B & $106.5(2)$ & $\mathrm{C} 7 \mathrm{~B}-\mathrm{N} 1 \mathrm{~B}-\mathrm{C} 1 \mathrm{~B}$ & $105.6(5)$ \\
\hline N1A-Ni1-N1B & $95.1(3)$ & $\mathrm{C} 7 \mathrm{~B}-\mathrm{N} 1 \mathrm{~B}-\mathrm{Ni1}$ & $112.2(4)$ \\
\hline $\mathrm{N} 2 \mathrm{~B}-\mathrm{Ni1}-\mathrm{N} 3 \mathrm{~A}$ & $102.9(2)$ & $\mathrm{C} 1 \mathrm{~B}-\mathrm{N} 1 \mathrm{~B}-\mathrm{Ni1}$ & $142.2(5)$ \\
\hline $\mathrm{N} 2 \mathrm{~A}-\mathrm{Ni} 1-\mathrm{N} 3 \mathrm{~A}$ & $77.2(2)$ & $\mathrm{C} 8 \mathrm{~B}-\mathrm{N} 2 \mathrm{~B}-\mathrm{C} 12 \mathrm{~B}$ & $121.3(6)$ \\
\hline $\mathrm{N} 1 \mathrm{~A}-\mathrm{Ni1}-\mathrm{N} 3 \mathrm{~A}$ & $154.9(2)$ & $\mathrm{C} 8 \mathrm{~B}-\mathrm{N} 2 \mathrm{~B}-\mathrm{Ni1}$ & $119.5(4)$ \\
\hline $\mathrm{N} 1 \mathrm{~B}-\mathrm{Ni1}-\mathrm{N} 3 \mathrm{~A}$ & $89.5(3)$ & $\mathrm{C} 12 \mathrm{~B}-\mathrm{N} 2 \mathrm{~B}-\mathrm{Ni1}$ & $119.2(4)$ \\
\hline $\mathrm{N} 2 \mathrm{~B}-\mathrm{Ni1}-\mathrm{N} 3 \mathrm{~B}$ & $77.5(2)$ & $\mathrm{C} 13 \mathrm{~B}-\mathrm{N} 3 \mathrm{~B}-\mathrm{C} 19 \mathrm{~B}$ & $104.5(5)$ \\
\hline $\mathrm{N} 2 \mathrm{~A}-\mathrm{Ni1}-\mathrm{N} 3 \mathrm{~B}$ & $98.5(2)$ & $\mathrm{C} 13 \mathrm{~B}-\mathrm{N} 3 \mathrm{~B}-\mathrm{Ni1}$ & $112.2(4)$ \\
\hline N1A-Ni1-N3B & $89.7(3)$ & $\mathrm{C} 19 \mathrm{~B}-\mathrm{N} 3 \mathrm{~B}-\mathrm{Ni1}$ & $143.2(5)$ \\
\hline $\mathrm{N} 1 \mathrm{~B}-\mathrm{Ni1}-\mathrm{N} 3 \mathrm{~B}$ & $155.0(2)$ & $\mathrm{C} 7 \mathrm{~B}-\mathrm{N} 4 \mathrm{~B}-\mathrm{C} 6 \mathrm{~B}$ & $107.0(5)$ \\
\hline $\mathrm{N} 3 \mathrm{~A}-\mathrm{Ni1}-\mathrm{N} 3 \mathrm{~B}$ & $96.5(2)$ & $\mathrm{C} 7 \mathrm{~B}-\mathrm{N} 4 \mathrm{~B}-\mathrm{H} 4 \mathrm{NB}$ & 126.5 \\
\hline $\mathrm{C} 7 \mathrm{~A}-\mathrm{N} 1 \mathrm{~A}-\mathrm{C} 1 \mathrm{~A}$ & $105.8(5)$ & $\mathrm{C} 6 \mathrm{~B}-\mathrm{N} 4 \mathrm{~B}-\mathrm{H} 4 \mathrm{NB}$ & 126.5 \\
\hline $\mathrm{C} 7 \mathrm{~A}-\mathrm{N} 1 \mathrm{~A}-\mathrm{Ni1}$ & $111.8(4)$ & $\mathrm{C} 13 \mathrm{~B}-\mathrm{N} 5 \mathrm{~B}-\mathrm{C} 14 \mathrm{~B}$ & $105.9(6)$ \\
\hline $\mathrm{C} 1 \mathrm{~A}-\mathrm{N} 1 \mathrm{~A}-\mathrm{Ni1}$ & $142.3(5)$ & $\mathrm{C} 13 \mathrm{~B}-\mathrm{N} 5 \mathrm{~B}-\mathrm{H} 5 \mathrm{NB}$ & 127.0 \\
\hline $\mathrm{C} 8 \mathrm{~A}-\mathrm{N} 2 \mathrm{~A}-\mathrm{C} 12 \mathrm{~A}$ & $121.8(5)$ & $\mathrm{C} 14 \mathrm{~B}-\mathrm{N} 5 \mathrm{~B}-\mathrm{H} 5 \mathrm{NB}$ & 127.0 \\
\hline $\mathrm{C} 8 \mathrm{~A}-\mathrm{N} 2 \mathrm{~A}-\mathrm{Ni1}$ & $118.8(4)$ & $\mathrm{C} 2 \mathrm{~B}-\mathrm{C} 1 \mathrm{~B}-\mathrm{N} 1 \mathrm{~B}$ & $131.0(7)$ \\
\hline $\mathrm{C} 12 \mathrm{~A}-\mathrm{N} 2 \mathrm{~A}-\mathrm{Ni1}$ & $119.0(4)$ & $\mathrm{C} 2 \mathrm{~B}-\mathrm{C} 1 \mathrm{~B}-\mathrm{C} 6 \mathrm{~B}$ & $120.9(6)$ \\
\hline $\mathrm{C} 13 \mathrm{~A}-\mathrm{N} 3 \mathrm{~A}-\mathrm{C} 19 \mathrm{~A}$ & $105.1(5)$ & $\mathrm{N} 1 \mathrm{~B}-\mathrm{C} 1 \mathrm{~B}-\mathrm{C} 6 \mathrm{~B}$ & $108.1(6)$ \\
\hline $\mathrm{C} 13 \mathrm{~A}-\mathrm{N} 3 \mathrm{~A}-\mathrm{Ni1}$ & $113.0(4)$ & $\mathrm{C} 3 \mathrm{~B}-\mathrm{C} 2 \mathrm{~B}-\mathrm{C} 1 \mathrm{~B}$ & $115.9(7)$ \\
\hline $\mathrm{C} 19 \mathrm{~A}-\mathrm{N} 3 \mathrm{~A}-\mathrm{Ni1}$ & $141.8(4)$ & $\mathrm{C} 3 \mathrm{~B}-\mathrm{C} 2 \mathrm{~B}-\mathrm{H} 2 \mathrm{BA}$ & 122.0 \\
\hline $\mathrm{C} 7 \mathrm{~A}-\mathrm{N} 4 \mathrm{~A}-\mathrm{C} 6 \mathrm{~A}$ & $107.1(5)$ & $\mathrm{C} 1 \mathrm{~B}-\mathrm{C} 2 \mathrm{~B}-\mathrm{H} 2 \mathrm{BA}$ & 122.0 \\
\hline $\mathrm{C} 7 \mathrm{~A}-\mathrm{N} 4 \mathrm{~A}-\mathrm{H} 4 \mathrm{NA}$ & 126.5 & $\mathrm{C} 4 \mathrm{~B}-\mathrm{C} 3 \mathrm{~B}-\mathrm{C} 2 \mathrm{~B}$ & $123.5(8)$ \\
\hline $\mathrm{C} 6 \mathrm{~A}-\mathrm{N} 4 \mathrm{~A}-\mathrm{H} 4 \mathrm{NA}$ & 126.5 & $\mathrm{C} 4 \mathrm{~B}-\mathrm{C} 3 \mathrm{~B}-\mathrm{H} 3 \mathrm{BA}$ & 118.2 \\
\hline $\mathrm{C} 13 \mathrm{~A}-\mathrm{N} 5 \mathrm{~A}-\mathrm{C} 14 \mathrm{~A}$ & $106.2(6)$ & $\mathrm{C} 2 \mathrm{~B}-\mathrm{C} 3 \mathrm{~B}-\mathrm{H} 3 \mathrm{BA}$ & 118.2 \\
\hline $\mathrm{C} 13 \mathrm{~A}-\mathrm{N} 5 \mathrm{~A}-\mathrm{H} 5 \mathrm{NA}$ & 126.9 & $\mathrm{C} 5 \mathrm{~B}-\mathrm{C} 4 \mathrm{~B}-\mathrm{C} 3 \mathrm{~B}$ & $121.4(8)$ \\
\hline $\mathrm{C} 14 \mathrm{~A}-\mathrm{N} 5 \mathrm{~A}-\mathrm{H} 5 \mathrm{NA}$ & 126.9 & $\mathrm{C} 5 \mathrm{~B}-\mathrm{C} 4 \mathrm{~B}-\mathrm{H} 4 \mathrm{BA}$ & 119.3 \\
\hline $\mathrm{C} 2 \mathrm{~A}-\mathrm{C} 1 \mathrm{~A}-\mathrm{N} 1 \mathrm{~A}$ & $130.5(6)$ & $\mathrm{C} 3 \mathrm{~B}-\mathrm{C} 4 \mathrm{~B}-\mathrm{H} 4 \mathrm{BA}$ & 119.3 \\
\hline $\mathrm{C} 2 \mathrm{~A}-\mathrm{C} 1 \mathrm{~A}-\mathrm{C} 6 \mathrm{~A}$ & $121.4(6)$ & $\mathrm{C} 4 \mathrm{~B}-\mathrm{C} 5 \mathrm{~B}-\mathrm{C} 6 \mathrm{~B}$ & $117.8(8)$ \\
\hline $\mathrm{N} 1 \mathrm{~A}-\mathrm{C} 1 \mathrm{~A}-\mathrm{C} 6 \mathrm{~A}$ & $108.0(6)$ & $\mathrm{C} 4 \mathrm{~B}-\mathrm{C} 5 \mathrm{~B}-\mathrm{H} 5 \mathrm{BA}$ & 121.1 \\
\hline $\mathrm{C} 3 \mathrm{~A}-\mathrm{C} 2 \mathrm{~A}-\mathrm{C} 1 \mathrm{~A}$ & $114.7(7)$ & $\mathrm{C} 6 \mathrm{~B}-\mathrm{C} 5 \mathrm{~B}-\mathrm{H} 5 \mathrm{BA}$ & 121.1 \\
\hline $\mathrm{C} 3 \mathrm{~A}-\mathrm{C} 2 \mathrm{~A}-\mathrm{H} 2 \mathrm{AA}$ & 122.6 & $\mathrm{~N} 4 \mathrm{~B}-\mathrm{C} 6 \mathrm{~B}-\mathrm{C} 5 \mathrm{~B}$ & $132.6(7)$ \\
\hline $\mathrm{C} 1 \mathrm{~A}-\mathrm{C} 2 \mathrm{~A}-\mathrm{H} 2 \mathrm{AA}$ & 122.6 & $\mathrm{~N} 4 \mathrm{~B}-\mathrm{C} 6 \mathrm{~B}-\mathrm{C} 1 \mathrm{~B}$ & $106.8(6)$ \\
\hline $\mathrm{C} 4 \mathrm{~A}-\mathrm{C} 3 \mathrm{~A}-\mathrm{C} 2 \mathrm{~A}$ & $123.9(8)$ & $\mathrm{C} 5 \mathrm{~B}-\mathrm{C} 6 \mathrm{~B}-\mathrm{C} 1 \mathrm{~B}$ & $120.5(7)$ \\
\hline
\end{tabular}




\begin{tabular}{|c|c|}
\hline $\mathrm{C} 4 \mathrm{~A}-\mathrm{C} 3 \mathrm{~A}-\mathrm{H} 3 \mathrm{AA}$ & 118.0 \\
\hline $\mathrm{C} 2 \mathrm{~A}-\mathrm{C} 3 \mathrm{~A}-\mathrm{H} 3 \mathrm{AA}$ & 118.0 \\
\hline $\mathrm{C} 5 \mathrm{~A}-\mathrm{C} 4 \mathrm{~A}-\mathrm{C} 3 \mathrm{~A}$ & $121.8(8)$ \\
\hline $\mathrm{C} 5 \mathrm{~A}-\mathrm{C} 4 \mathrm{~A}-\mathrm{H} 4 \mathrm{AA}$ & 119.1 \\
\hline $\mathrm{C} 3 \mathrm{~A}-\mathrm{C} 4 \mathrm{~A}-\mathrm{H} 4 \mathrm{AA}$ & 119.1 \\
\hline $\mathrm{C} 4 \mathrm{~A}-\mathrm{C} 5 \mathrm{~A}-\mathrm{C} 6 \mathrm{~A}$ & $116.9(8)$ \\
\hline $\mathrm{C} 4 \mathrm{~A}-\mathrm{C} 5 \mathrm{~A}-\mathrm{H} 5 \mathrm{AA}$ & 121.5 \\
\hline $\mathrm{C} 6 \mathrm{~A}-\mathrm{C} 5 \mathrm{~A}-\mathrm{H} 5 \mathrm{AA}$ & 121.5 \\
\hline $\mathrm{N} 4 \mathrm{~A}-\mathrm{C} 6 \mathrm{~A}-\mathrm{C} 5 \mathrm{~A}$ & $132.2(7)$ \\
\hline $\mathrm{N} 4 \mathrm{~A}-\mathrm{C} 6 \mathrm{~A}-\mathrm{C} 1 \mathrm{~A}$ & $106.7(6)$ \\
\hline $\mathrm{C} 5 \mathrm{~A}-\mathrm{C} 6 \mathrm{~A}-\mathrm{C} 1 \mathrm{~A}$ & $121.1(7)$ \\
\hline $\mathrm{N} 1 \mathrm{~A}-\mathrm{C} 7 \mathrm{~A}-\mathrm{N} 4 \mathrm{~A}$ & $112.3(5)$ \\
\hline $\mathrm{N} 1 \mathrm{~A}-\mathrm{C} 7 \mathrm{~A}-\mathrm{C} 8 \mathrm{~A}$ & $119.5(6)$ \\
\hline $\mathrm{N} 4 \mathrm{~A}-\mathrm{C} 7 \mathrm{~A}-\mathrm{C} 8 \mathrm{~A}$ & $127.8(6)$ \\
\hline $\mathrm{N} 2 \mathrm{~A}-\mathrm{C} 8 \mathrm{~A}-\mathrm{C} 9 \mathrm{~A}$ & $120.6(6)$ \\
\hline $\mathrm{N} 2 \mathrm{~A}-\mathrm{C} 8 \mathrm{~A}-\mathrm{C} 7 \mathrm{~A}$ & $111.7(6)$ \\
\hline $\mathrm{C} 9 \mathrm{~A}-\mathrm{C} 8 \mathrm{~A}-\mathrm{C} 7 \mathrm{~A}$ & $127.7(6)$ \\
\hline $\mathrm{C} 10 \mathrm{~A}-\mathrm{C} 9 \mathrm{~A}-\mathrm{C} 8 \mathrm{~A}$ & $118.2(7)$ \\
\hline $\mathrm{C} 10 \mathrm{~A}-\mathrm{C} 9 \mathrm{~A}-\mathrm{H} 9 \mathrm{AA}$ & 120.9 \\
\hline C8A-C9A-H9AA & 120.9 \\
\hline $\mathrm{C} 9 \mathrm{~A}-\mathrm{C} 10 \mathrm{~A}-\mathrm{C} 11 \mathrm{~A}$ & $121.6(7)$ \\
\hline $\mathrm{C} 9 \mathrm{~A}-\mathrm{C} 10 \mathrm{~A}-\mathrm{H} 10 \mathrm{~A}$ & 119.2 \\
\hline $\mathrm{C} 11 \mathrm{~A}-\mathrm{C} 10 \mathrm{~A}-\mathrm{H} 10 \mathrm{~A}$ & 119.2 \\
\hline $\mathrm{C} 12 \mathrm{~A}-\mathrm{C} 11 \mathrm{~A}-\mathrm{C} 10 \mathrm{~A}$ & $117.4(7)$ \\
\hline $\mathrm{C} 12 \mathrm{~A}-\mathrm{C} 11 \mathrm{~A}-\mathrm{H} 11 \mathrm{~A}$ & 121.3 \\
\hline $\mathrm{C} 10 \mathrm{~A}-\mathrm{C} 11 \mathrm{~A}-\mathrm{H} 11 \mathrm{~A}$ & 121.3 \\
\hline $\mathrm{N} 2 \mathrm{~A}-\mathrm{C} 12 \mathrm{~A}-\mathrm{C} 11 \mathrm{~A}$ & $120.4(6)$ \\
\hline $\mathrm{N} 2 \mathrm{~A}-\mathrm{C} 12 \mathrm{~A}-\mathrm{C} 13 \mathrm{~A}$ & $111.3(5)$ \\
\hline $\mathrm{C} 11 \mathrm{~A}-\mathrm{C} 12 \mathrm{~A}-\mathrm{C} 13 \mathrm{~A}$ & $128.3(6)$ \\
\hline $\mathrm{N} 3 \mathrm{~A}-\mathrm{C} 13 \mathrm{~A}-\mathrm{N} 5 \mathrm{~A}$ & $113.2(5)$ \\
\hline $\mathrm{N} 3 \mathrm{~A}-\mathrm{C} 13 \mathrm{~A}-\mathrm{C} 12 \mathrm{~A}$ & $118.9(5)$ \\
\hline $\mathrm{N} 5 \mathrm{~A}-\mathrm{C} 13 \mathrm{~A}-\mathrm{C} 12 \mathrm{~A}$ & $127.7(6)$ \\
\hline $\mathrm{C} 19 \mathrm{~A}-\mathrm{C} 14 \mathrm{~A}-\mathrm{N} 5 \mathrm{~A}$ & $106.6(6)$ \\
\hline $\mathrm{C} 19 \mathrm{~A}-\mathrm{C} 14 \mathrm{~A}-\mathrm{C} 15 \mathrm{~A}$ & $122.3(7)$ \\
\hline $\mathrm{N} 5 \mathrm{~A}-\mathrm{C} 14 \mathrm{~A}-\mathrm{C} 15 \mathrm{~A}$ & $131.1(7)$ \\
\hline $\mathrm{C} 16 \mathrm{~A}-\mathrm{C} 15 \mathrm{~A}-\mathrm{C} 14 \mathrm{~A}$ & $115.9(8)$ \\
\hline $\mathrm{C} 16 \mathrm{~A}-\mathrm{C} 15 \mathrm{~A}-\mathrm{H} 15 \mathrm{~A}$ & 122.0 \\
\hline $\mathrm{C} 14 \mathrm{~A}-\mathrm{C} 15 \mathrm{~A}-\mathrm{H} 15 \mathrm{~A}$ & 122.0 \\
\hline $\mathrm{C} 15 \mathrm{~A}-\mathrm{C} 16 \mathrm{~A}-\mathrm{C} 17 \mathrm{~A}$ & $122.9(8)$ \\
\hline $\mathrm{C} 15 \mathrm{~A}-\mathrm{C} 16 \mathrm{~A}-\mathrm{H} 16 \mathrm{~A}$ & 118.6 \\
\hline $\mathrm{C} 17 \mathrm{~A}-\mathrm{C} 16 \mathrm{~A}-\mathrm{H} 16 \mathrm{~A}$ & 118.6 \\
\hline $\mathrm{C} 18 \mathrm{~A}-\mathrm{C} 17 \mathrm{~A}-\mathrm{C} 16 \mathrm{~A}$ & $121.3(8)$ \\
\hline $\mathrm{C} 18 \mathrm{~A}-\mathrm{C} 17 \mathrm{~A}-\mathrm{H} 17 \mathrm{~A}$ & 119.3 \\
\hline $\mathrm{C} 16 \mathrm{~A}-\mathrm{C} 17 \mathrm{~A}-\mathrm{H} 17 \mathrm{~A}$ & 119.3 \\
\hline $\mathrm{C} 17 \mathrm{~A}-\mathrm{C} 18 \mathrm{~A}-\mathrm{C} 19 \mathrm{~A}$ & $116.9(7)$ \\
\hline $\mathrm{C} 17 \mathrm{~A}-\mathrm{C} 18 \mathrm{~A}-\mathrm{H} 18 \mathrm{~A}$ & 121.5 \\
\hline
\end{tabular}

$112.6(5)$

$119.0(6)$

$128.4(6)$

$120.8(6)$

$111.7(6)$

$127.5(6)$

$118.4(7)$

120.8

120.8

$121.2(8)$

119.4

119.4

$117.2(7)$

121.4

121.4

$120.9(6)$

$111.5(5)$

$127.6(6)$

$114.0(5)$

$119.5(6)$

$126.5(6)$

$106.5(6)$

$122.5(7)$

$130.9(7)$

$116.3(8)$

121.8

121.8

$122.3(8)$

118.8

118.8

$121.6(8)$

119.2

119.2

117.0 (7)

121.5

121.5

120.1 (7)

$109.1(6)$

130.7 (7)

$108.6(4)$

110.3 (4)

109.7 (4)

108.8 (4)

111.4 (4)

108.1 (4) 
Hydrogen-bond geometry $\left(\AA,{ }^{\circ}\right)$

\begin{tabular}{lllll}
\hline$D-\mathrm{H}^{\cdots} \cdot A$ & $D-\mathrm{H}$ & $\mathrm{H} \cdots A$ & $D \cdots A$ & $D-\mathrm{H} \cdots A$ \\
\hline $\mathrm{N} 4 A-\mathrm{H} 4 N A \cdots \mathrm{O} 2^{\mathrm{i}}$ & 0.86 & 1.82 & $2.654(8)$ & 163 \\
$\mathrm{~N} 5 A-\mathrm{H} 5 N A \cdots \mathrm{O} 1^{\mathrm{ii}}$ & 0.86 & 1.85 & $2.701(8)$ & 171 \\
$\mathrm{~N} 4 B-\mathrm{H} 4 N B \cdots \mathrm{O} 4^{i i i}$ & 0.86 & 1.85 & $2.685(9)$ & 164 \\
$\mathrm{~N} 5 B-\mathrm{H} 5 N B \cdots \mathrm{O} 3$ & 0.86 & 1.81 & $2.663(9)$ & 173
\end{tabular}

Symmetry codes: (i) $-x+3 / 2,-y, z-1 / 2$; (ii) $-x+5 / 2,-y, z-1 / 2$; (iii) $x-1 / 2,-y+1 / 2,-z+2$.

Bis $\left[2,6\right.$-bis $\left(1 H\right.$-benzimidazol-2-yl- $\left.\kappa N^{3}\right)$ pyridine- $\left.\kappa N\right]$ nickel(II) sulfate (II)

Crystal data

$\left[\mathrm{Ni}\left(\mathrm{C}_{19} \mathrm{H}_{13} \mathrm{~N}_{5}\right)_{2}\right] \mathrm{SO}_{4}$

$M_{r}=777.46$

Tetragonal, $I \overline{4}$

$a=28.596(2) \AA$

$c=13.5438(9) \AA$

$V=11075.0(17) \AA^{3}$

$Z=8$

$F(000)=3200$

\section{Data collection}

Oxford Diffraction Gemini CCD S Ultra diffractometer

Radiation source: fine-focus sealed tube Graphite monochromator

$\omega$ scans, thick slices

Absorption correction: multi-scan

(CrysAlis PRO; Oxford Diffraction, 2009)

$T_{\min }=0.96, T_{\max }=0.98$

\section{Refinement}

Refinement on $F^{2}$

Least-squares matrix: full

$R\left[F^{2}>2 \sigma\left(F^{2}\right)\right]=0.078$

$w R\left(F^{2}\right)=0.220$

$S=0.89$

11374 reflections

488 parameters

586 restraints
$D_{\mathrm{x}}=0.933 \mathrm{Mg} \mathrm{m}^{-3}$

Mo $K \alpha$ radiation, $\lambda=0.71073 \AA$

Cell parameters from 3126 reflections

$\theta=4.0-24.9^{\circ}$

$\mu=0.43 \mathrm{~mm}^{-1}$

$T=294 \mathrm{~K}$

Needles, light red

$0.38 \times 0.14 \times \overline{0} .12 \mathrm{~mm}$

16294 measured reflections

11374 independent reflections

4172 reflections with $I>2 \sigma(I)$

$R_{\text {int }}=0.095$

$\theta_{\max }=28.9^{\circ}, \theta_{\min }=3.6^{\circ}$

$h=-31 \rightarrow 33$

$k=-38 \rightarrow 37$

$l=-18 \rightarrow 9$

Hydrogen site location: inferred from neighbouring sites

$\mathrm{H}$-atom parameters constrained

$w=1 /\left[\sigma^{2}\left(F_{\mathrm{o}}^{2}\right)+(0.0593 P)^{2}\right]$

where $P=\left(F_{\mathrm{o}}^{2}+2 F_{\mathrm{c}}^{2}\right) / 3$

$(\Delta / \sigma)_{\max }<0.001$

$\Delta \rho_{\max }=0.55$ e $\AA^{-3}$

$\Delta \rho_{\min }=-0.23$ e $\AA^{-3}$

Absolute structure: Refined as an inversion twin

Absolute structure parameter: 0.45 (3)

Special details

Geometry. All e.s.d.'s (except the e.s.d. in the dihedral angle between two 1.s. planes) are estimated using the full covariance matrix. The cell e.s.d.'s are taken into account individually in the estimation of e.s.d.'s in distances, angles and torsion angles; correlations between e.s.d.'s in cell parameters are only used when they are defined by crystal symmetry. An approximate (isotropic) treatment of cell e.s.d.'s is used for estimating e.s.d.'s involving l.s. planes.

Refinement. Refined as a 2-component inversion twin 
Fractional atomic coordinates and isotropic or equivalent isotropic displacement parameters $\left(\AA^{2}\right)$

\begin{tabular}{|c|c|c|c|c|}
\hline & $x$ & $y$ & $z$ & $U_{\text {iso }} * / U_{\text {eq }}$ \\
\hline Nil & $0.64892(5)$ & $0.18669(5)$ & $0.10607(8)$ & 0.0538 \\
\hline N1A & $0.5815(2)$ & $0.2131(3)$ & $0.1423(5)$ & $0.062(2)$ \\
\hline $\mathrm{N} 2 \mathrm{~A}$ & $0.6099(2)$ & $0.1636(3)$ & $-0.0099(5)$ & 0.0531 (19) \\
\hline N3A & $0.6974(2)$ & 0.1497 & $0.0178(5)$ & $0.061(2)$ \\
\hline $\mathrm{N} 4 \mathrm{~A}$ & 0.5075 & 0.2189 & $0.0920(5)$ & $0.075(2)$ \\
\hline H4NA & 0.483254 & 0.217136 & 0.054487 & $0.089^{*}$ \\
\hline N5A & 0.7103 & 0.1091 & $-0.1203(5)$ & $0.068(2)$ \\
\hline H5NA & 0.704557 & 0.094781 & -0.174876 & $0.082^{*}$ \\
\hline $\mathrm{C} 1 \mathrm{~A}$ & 0.5558 & $0.2366(4)$ & $0.2124(6)$ & $0.078(3)$ \\
\hline $\mathrm{C} 2 \mathrm{~A}$ & $0.5689(4)$ & $0.2539(5)$ & $0.3048(7)$ & $0.116(5)$ \\
\hline H2AA & 0.599239 & 0.250325 & 0.328178 & $0.139 *$ \\
\hline $\mathrm{C} 3 \mathrm{~A}$ & $0.5360(4)$ & $0.2759(6)$ & $0.3593(8)$ & $0.126(5)$ \\
\hline H3AA & 0.544595 & 0.290804 & 0.417519 & $0.151^{*}$ \\
\hline $\mathrm{C} 4 \mathrm{~A}$ & $0.4893(5)$ & $0.2765(8)$ & $0.3294(10)$ & $0.190(9)$ \\
\hline H4AA & 0.467164 & 0.287917 & 0.373500 & $0.228^{*}$ \\
\hline C5A & $0.4733(4)$ & $0.2606(6)$ & $0.2344(9)$ & $0.138(6)$ \\
\hline H5AA & 0.442980 & 0.264417 & 0.211100 & $0.165^{*}$ \\
\hline C6A & 0.5091 & 0.2388 & $0.1817(7)$ & $0.084(3)$ \\
\hline C7A & $0.5506(3)$ & $0.2021(4)$ & $0.0710(6)$ & $0.056(2)$ \\
\hline C8A & $0.5646(3)$ & $0.1756(3)$ & $-0.0156(6)$ & $0.056(2)$ \\
\hline C9A & 0.5358 & $0.1621(4)$ & $-0.0931(6)$ & $0.079(3)$ \\
\hline H9AA & 0.504281 & 0.170180 & -0.095187 & $0.094^{*}$ \\
\hline $\mathrm{C} 10 \mathrm{~A}$ & $0.5563(4)$ & $0.1363(5)$ & $-0.1659(7)$ & $0.091(4)$ \\
\hline $\mathrm{H} 10 \mathrm{~A}$ & 0.538173 & 0.125808 & -0.218326 & $0.109 *$ \\
\hline C11A & 0.6050 & $0.1251(4)$ & $-0.1639(7)$ & $0.079(3)$ \\
\hline H11A & 0.619238 & 0.109115 & -0.215507 & $0.095 *$ \\
\hline $\mathrm{C} 12 \mathrm{~A}$ & $0.6297(3)$ & $0.1391(3)$ & $-0.0818(6)$ & $0.055(2)$ \\
\hline C13A & 0.6780 & 0.1318 & $-0.0631(6)$ & 0.057 (2) \\
\hline C14A & 0.7533 & $0.1129(4)$ & $-0.0763(6)$ & $0.070(3)$ \\
\hline $\mathrm{C} 15 \mathrm{~A}$ & $0.7965(3)$ & $0.1010(5)$ & $-0.1081(8)$ & $0.094(4)$ \\
\hline $\mathrm{H} 15 \mathrm{~A}$ & 0.801520 & 0.086460 & -0.168601 & $0.112^{*}$ \\
\hline C16A & $0.8319(4)$ & $0.1116(5)$ & $-0.0462(9)$ & $0.114(5)$ \\
\hline H16A & 0.862131 & 0.102871 & -0.063832 & $0.137^{*}$ \\
\hline C17A & $0.8253(4)$ & $0.1354(6)$ & $0.0443(8)$ & $0.116(5)$ \\
\hline H17A & 0.850964 & 0.142010 & 0.084021 & $0.139^{*}$ \\
\hline C18A & 0.7822 & $0.1485(4)$ & $0.0737(7)$ & $0.080(3)$ \\
\hline $\mathrm{H} 18 \mathrm{~A}$ & 0.777337 & 0.164500 & 0.132678 & $0.096^{*}$ \\
\hline C19A & 0.7450 & $0.1369(4)$ & $0.0108(6)$ & $0.065(3)$ \\
\hline N1B & 0.6459 & $0.1332(2)$ & $0.2173(5)$ & $0.0525(18)$ \\
\hline $\mathrm{N} 2 \mathrm{~B}$ & $0.6829(3)$ & $0.2170(2)$ & $0.2206(5)$ & 0.0533 (19) \\
\hline N3B & 0.6708 & 0.2514 & $0.0476(5)$ & $0.063(2)$ \\
\hline N4B & 0.6554 & 0.1188 & $0.3777(5)$ & $0.068(2)$ \\
\hline H4NB & 0.663031 & 0.122576 & 0.438651 & $0.081^{*}$ \\
\hline N5B & 0.7053 & $0.3203(3)$ & $0.0782(5)$ & $0.067(2)$ \\
\hline H5NB & 0.719367 & 0.341965 & 0.110748 & $0.080^{*}$ \\
\hline
\end{tabular}




$\begin{array}{lllll}\text { C1B } & 0.6283(3) & 0.0894(3) & 0.2377(6) & 0.058(2) \\ \text { C2B } & 0.6095(4) & 0.0554(3) & 0.1762(7) & 0.082(3) \\ \text { H2BA } & 0.607350 & 0.060309 & 0.108477 & 0.098^{*} \\ \text { C3B } & 0.5944(5) & 0.0151(4) & 0.2172(9) & 0.104(4) \\ \text { H3BA } & 0.581404 & -0.007986 & 0.177407 & 0.125^{*} \\ \text { C4B } & 0.5983(5) & 0.0078(4) & 0.3186(9) & 0.120(5) \\ \text { H4BA } & 0.587447 & -0.020378 & 0.344123 & 0.144^{*} \\ \text { C5B } & 0.6179(4) & 0.0410(3) & 0.3860(8) & 0.086(3) \\ \text { H5BA } & 0.619811 & 0.036275 & 0.453831 & 0.103^{*} \\ \text { C6B } & 0.6339(4) & 0.0811(3) & 0.3389(6) & 0.070(3) \\ \text { C7B } & 0.6630(4) & 0.1495(3) & 0.3036(6) & 0.064(3) \\ \text { C8B } & 0.6850(4) & 0.1950(3) & 0.3084(6) & 0.063(3) \\ \text { C9B } & 0.7042(4) & 0.2157(3) & 0.3924(7) & 0.076(3) \\ \text { H9BA } & 0.703975 & 0.200865 & 0.453502 & 0.091^{*} \\ \text { C10B } & 0.7233(4) & 0.2590(4) & 0.3793(7) & 0.094(4) \\ \text { H10B } & 0.737897 & 0.273297 & 0.432737 & 0.113^{*} \\ \text { C11B } & 0.7220(4) & 0.2832(3) & 0.2871(7) & 0.077(3) \\ \text { H11B } & 0.734631 & 0.312904 & 0.279180 & 0.093^{*} \\ \text { C12B } & 0.7006(4) & 0.2597(3) & 0.2109(6) & 0.061(3) \\ \text { C13B } & 0.6930(4) & 0.2774(3) & 0.1145(6) & 0.064(3) \\ \text { C14B } & 0.6915(4) & 0.3231(3) & -0.0184(7) & 0.074(3) \\ \text { C15B } & 0.6943(4) & 0.3587(4) & -0.0847(7) & 0.090(3) \\ \text { H15B } & 0.708964 & 0.386891 & -0.070613 & 0.108^{*} \\ \text { C16B } & 0.6739(5) & 0.3497(4) & -0.1730(8) & 0.100(4) \\ \text { H16B } & 0.673751 & 0.373328 & -0.220207 & 0.120^{*} \\ \text { C17B } & 0.6529(5) & 0.3064(4) & -0.1973(7) & 0.094(4) \\ \text { H17B } & 0.641722 & 0.301502 & -0.260922 & 0.113^{*} \\ \text { C18B } & 0.6489(4) & 0.2721(3) & -0.1290(6) & 0.076(3) \\ \text { H18B } & 0.634138 & 0.243910 & -0.143115 & 0.092^{*} \\ \text { C19B } & 0.6683(3) & 0.2813(3) & -0.0361(6) & 0.060(2) \\ \text { S1 } & 0.79506(12) & 0.40472(10) & 0.1171(2) & 0.0672(8) \\ \text { O1 } & 0.7526(3) & 0.3852(3) & 0.1690(5) & 0.099(3) \\ \text { O2 } & 0.8230(3) & 0.4275(3) & 0.1943(5) & 0.086(2) \\ \text { O3 } & 0.7816(3) & 0.4362(3) & 0.0395(5) & 0.095(3) \\ \text { O4 } & 0.8206(4) & 0.3659(3) & 0.0714(5) & 0.110(3) \\ & & & & \end{array}$

Atomic displacement parameters $\left(\AA^{2}\right)$

\begin{tabular}{lllllll}
\hline & $U^{11}$ & $U^{22}$ & $U^{33}$ & $U^{12}$ & $U^{13}$ & $U^{23}$ \\
\hline Ni1 & $0.0611(9)$ & $0.0644(9)$ & $0.0361(6)$ & $-0.0025(7)$ & $-0.0033(7)$ & $-0.0036(7)$ \\
N1A & $0.068(3)$ & $0.079(5)$ & $0.040(3)$ & $-0.001(2)$ & $-0.0065(16)$ & $-0.008(3)$ \\
N2A & $0.062(2)$ & $0.062(4)$ & $0.036(2)$ & $-0.004(2)$ & $-0.001(2)$ & $-0.001(3)$ \\
N3A & $0.069(3)$ & $0.073(4)$ & $0.042(3)$ & $0.000(3)$ & $-0.0023(18)$ & $-0.007(3)$ \\
N4A & $0.067(3)$ & $0.105(6)$ & $0.052(4)$ & $0.009(3)$ & $-0.003(2)$ & $-0.017(4)$ \\
N5A & $0.076(3)$ & $0.086(6)$ & $0.042(3)$ & $0.011(3)$ & $0.002(2)$ & $-0.004(3)$ \\
C1A & $0.085(4)$ & $0.103(7)$ & $0.046(3)$ & $0.015(4)$ & $-0.005(2)$ & $-0.014(4)$ \\
C2A & $0.106(6)$ & $0.176(11)$ & $0.066(4)$ & $0.048(7)$ & $-0.024(4)$ & $-0.052(5)$ \\
C3A & $0.117(5)$ & $0.182(13)$ & $0.078(7)$ & $0.053(7)$ & $-0.014(4)$ & $-0.051(7)$
\end{tabular}




\begin{tabular}{|c|c|c|c|c|c|c|}
\hline $\mathrm{C} 4 \mathrm{~A}$ & $0.120(5)$ & $0.34(2)$ & $0.107(7)$ & $0.074(8)$ & $-0.023(5)$ & $-0.112(10)$ \\
\hline C5A & $0.106(5)$ & $0.221(14)$ & $0.085(6)$ & $0.055(7)$ & $-0.011(4)$ & $-0.063(7)$ \\
\hline C6A & $0.086(4)$ & $0.113(8)$ & $0.054(4)$ & $0.018(4)$ & -0.008 & -0.021 \\
\hline C7A & $0.063(3)$ & $0.071(5)$ & 0.035 & -0.001 & $-0.002(2)$ & -0.005 \\
\hline $\mathrm{C} 8 \mathrm{~A}$ & $0.062(2)$ & $0.069(5)$ & $0.035(3)$ & $-0.002(3)$ & $-0.002(2)$ & $-0.004(3)$ \\
\hline C9A & $0.078(4)$ & $0.110(8)$ & $0.048(3)$ & $-0.005(4)$ & -0.013 & $-0.017(4)$ \\
\hline $\mathrm{C} 10 \mathrm{~A}$ & $0.086(4)$ & $0.131(9)$ & $0.055(5)$ & $0.003(5)$ & -0.014 & $-0.028(5)$ \\
\hline C11A & $0.084(4)$ & $0.103(9)$ & $0.050(3)$ & -0.003 & $-0.011(3)$ & -0.015 \\
\hline $\mathrm{C} 12 \mathrm{~A}$ & $0.066(2)$ & $0.062(5)$ & $0.037(3)$ & $-0.003(3)$ & 0.0007 (19) & -0.001 \\
\hline $\mathrm{C} 13 \mathrm{~A}$ & $0.066(2)$ & $0.067(6)$ & $0.039(3)$ & $-0.002(3)$ & $0.000(2)$ & $-0.004(3)$ \\
\hline C14A & $0.077(3)$ & $0.092(7)$ & $0.041(3)$ & $0.006(3)$ & $0.001(2)$ & $0.004(4)$ \\
\hline $\mathrm{C} 15 \mathrm{~A}$ & $0.079(3)$ & $0.140(11)$ & $0.062(5)$ & $0.012(4)$ & $0.002(3)$ & $-0.018(6)$ \\
\hline $\mathrm{C} 16 \mathrm{~A}$ & $0.083(4)$ & $0.178(12)$ & $0.081(5)$ & $0.020(6)$ & $-0.006(4)$ & $-0.041(6)$ \\
\hline C17A & $0.081(3)$ & $0.187(12)$ & $0.080(5)$ & $0.039(6)$ & $-0.017(4)$ & $-0.043(6)$ \\
\hline C18A & $0.076(3)$ & $0.097(9)$ & $0.067(5)$ & $0.020(4)$ & -0.013 & $-0.016(5)$ \\
\hline C19A & $0.070(3)$ & $0.086(6)$ & $0.040(3)$ & $0.005(2)$ & $0.0014(18)$ & $0.007(4)$ \\
\hline N1B & $0.056(5)$ & 0.060 & $0.042(2)$ & $0.001(2)$ & $0.002(2)$ & $-0.004(2)$ \\
\hline N2B & $0.063(4)$ & $0.056(3)$ & $0.041(2)$ & $0.002(3)$ & $-0.005(3)$ & $-0.0037(16)$ \\
\hline N3B & $0.072(5)$ & $0.072(3)$ & $0.045(2)$ & $-0.006(3)$ & $-0.006(3)$ & $0.001(2)$ \\
\hline N4B & $0.076(6)$ & $0.073(3)$ & $0.054(3)$ & $-0.007(3)$ & $-0.011(4)$ & 0.009 (2) \\
\hline N5B & $0.078(6)$ & $0.069(3)$ & $0.053(3)$ & $-0.004(3)$ & $-0.005(3)$ & $0.004(2)$ \\
\hline C1B & $0.053(5)$ & $0.061(3)$ & $0.059(3)$ & $0.000(3)$ & $-0.005(3)$ & $0.002(2)$ \\
\hline $\mathrm{C} 2 \mathrm{~B}$ & $0.103(8)$ & $0.068(4)$ & $0.073(4)$ & $-0.010(4)$ & $-0.025(5)$ & $0.001(3)$ \\
\hline C3B & $0.131(11)$ & $0.074(5)$ & $0.107(4)$ & $-0.013(6)$ & $-0.009(6)$ & $0.011(4)$ \\
\hline $\mathrm{C} 4 \mathrm{~B}$ & $0.154(13)$ & $0.098(7)$ & $0.108(4)$ & $-0.029(7)$ & $-0.013(6)$ & $0.016(4)$ \\
\hline C5B & $0.084(7)$ & $0.081(4)$ & $0.094(5)$ & $-0.004(4)$ & $0.004(6)$ & 0.019 (3) \\
\hline $\mathrm{C} 6 \mathrm{~B}$ & $0.075(7)$ & $0.075(4)$ & $0.060(3)$ & $-0.008(4)$ & -0.009 (3) & $0.005(2)$ \\
\hline C7B & $0.086(6)$ & $0.060(3)$ & $0.045(3)$ & $-0.006(4)$ & -0.008 & $-0.002(2)$ \\
\hline $\mathrm{C} 8 \mathrm{~B}$ & $0.087(7)$ & $0.061(3)$ & $0.043(2)$ & $-0.006(3)$ & $-0.010(3)$ & $-0.001(2)$ \\
\hline C9B & $0.099(7)$ & $0.080(4)$ & $0.048(3)$ & $-0.011(5)$ & $-0.013(4)$ & -0.008 \\
\hline C10B & $0.128(10)$ & $0.089(5)$ & $0.065(4)$ & $-0.028(5)$ & $-0.037(5)$ & $0.005(4)$ \\
\hline C11B & $0.105(8)$ & $0.069(5)$ & $0.058(3)$ & $-0.006(5)$ & $-0.025(4)$ & $-0.007(3)$ \\
\hline C12B & $0.079(6)$ & $0.059(3)$ & $0.045(2)$ & $-0.006(3)$ & -0.010 & $-0.001(2)$ \\
\hline C13B & $0.078(6)$ & 0.069 (3) & $0.046(2)$ & $-0.006(3)$ & $-0.010(3)$ & $0.0026(19)$ \\
\hline C14B & $0.077(7)$ & $0.091(4)$ & $0.054(3)$ & $-0.015(4)$ & $-0.005(4)$ & $0.010(2)$ \\
\hline C15B & $0.114(9)$ & 0.095 (4) & $0.062(4)$ & $-0.004(5)$ & $0.011(4)$ & $0.015(3)$ \\
\hline C16B & $0.141(10)$ & $0.093(5)$ & $0.066(4)$ & $-0.014(6)$ & $0.000(6)$ & $0.020(4)$ \\
\hline C17B & $0.126(10)$ & $0.088(5)$ & 0.069 (4) & -0.004 (5) & $-0.010(6)$ & 0.024 (4) \\
\hline C18B & $0.095(8)$ & $0.078(5)$ & $0.057(3)$ & $-0.009(5)$ & $-0.019(4)$ & $0.013(3)$ \\
\hline C19B & $0.048(5)$ & $0.083(4)$ & $0.050(2)$ & $0.000(3)$ & $0.000(3)$ & $0.008(2)$ \\
\hline $\mathrm{S} 1$ & $0.102(2)$ & $0.0547(18)$ & $0.0452(15)$ & $-0.0061(17)$ & $-0.0069(17)$ & $0.0074(15)$ \\
\hline $\mathrm{O} 1$ & $0.124(4)$ & $0.104(7)$ & $0.070(4)$ & $-0.041(5)$ & $0.020(4)$ & $-0.007(4)$ \\
\hline $\mathrm{O} 2$ & $0.121(5)$ & $0.067(5)$ & $0.070(4)$ & $0.002(4)$ & $-0.032(5)$ & $-0.011(4)$ \\
\hline $\mathrm{O} 3$ & $0.137(7)$ & $0.082(4)$ & $0.065(4)$ & $0.002(5)$ & $-0.015(4)$ & $0.033(5)$ \\
\hline $\mathrm{O} 4$ & $0.181(7)$ & $0.071(4)$ & $0.077(5)$ & $0.032(5)$ & $0.028(5)$ & $0.007(4)$ \\
\hline
\end{tabular}


Geometric parameters $\left(A,{ }^{o}\right)$

\begin{tabular}{|c|c|c|c|}
\hline Ni1-N2B & $2.026(6)$ & C18A-H18A & 0.9300 \\
\hline $\mathrm{Ni1}-\mathrm{N} 2 \mathrm{~A}$ & $2.037(6)$ & $\mathrm{N} 1 \mathrm{~B}-\mathrm{C} 7 \mathrm{~B}$ & $1.350(8)$ \\
\hline Ni1-N3B & $2.109(7)$ & $\mathrm{N} 1 \mathrm{~B}-\mathrm{C} 1 \mathrm{~B}$ & $1.378(9)$ \\
\hline $\mathrm{Ni} 1-\mathrm{N} 3 \mathrm{~A}$ & $2.115(7)$ & $\mathrm{N} 2 \mathrm{~B}-\mathrm{C} 12 \mathrm{~B}$ & $1.327(9)$ \\
\hline Nil-N1A & $2.128(7)$ & $\mathrm{N} 2 \mathrm{~B}-\mathrm{C} 8 \mathrm{~B}$ & $1.346(8)$ \\
\hline Ni1-N1B & $2.149(7)$ & $\mathrm{N} 3 \mathrm{~B}-\mathrm{C} 13 \mathrm{~B}$ & $1.332(8)$ \\
\hline $\mathrm{N} 1 \mathrm{~A}-\mathrm{C} 7 \mathrm{~A}$ & $1.346(8)$ & $\mathrm{N} 3 \mathrm{~B}-\mathrm{C} 19 \mathrm{~B}$ & $1.421(9)$ \\
\hline $\mathrm{N} 1 \mathrm{~A}-\mathrm{C} 1 \mathrm{~A}$ & $1.375(9)$ & $\mathrm{N} 4 \mathrm{~B}-\mathrm{C} 7 \mathrm{~B}$ & $1.351(9)$ \\
\hline $\mathrm{N} 2 \mathrm{~A}-\mathrm{C} 12 \mathrm{~A}$ & $1.326(8)$ & $\mathrm{N} 4 \mathrm{~B}-\mathrm{C} 6 \mathrm{~B}$ & $1.349(9)$ \\
\hline $\mathrm{N} 2 \mathrm{~A}-\mathrm{C} 8 \mathrm{~A}$ & $1.343(9)$ & $\mathrm{N} 4 \mathrm{~B}-\mathrm{H} 4 \mathrm{NB}$ & 0.8600 \\
\hline $\mathrm{N} 3 \mathrm{~A}-\mathrm{C} 13 \mathrm{~A}$ & $1.330(8)$ & $\mathrm{N} 5 \mathrm{~B}-\mathrm{C} 13 \mathrm{~B}$ & $1.369(9)$ \\
\hline $\mathrm{N} 3 \mathrm{~A}-\mathrm{C} 19 \mathrm{~A}$ & $1.413(9)$ & $\mathrm{N} 5 \mathrm{~B}-\mathrm{C} 14 \mathrm{~B}$ & $1.369(9)$ \\
\hline $\mathrm{N} 4 \mathrm{~A}-\mathrm{C} 6 \mathrm{~A}$ & $1.342(9)$ & $\mathrm{N} 5 \mathrm{~B}-\mathrm{H} 5 \mathrm{NB}$ & 0.8600 \\
\hline $\mathrm{N} 4 \mathrm{~A}-\mathrm{C} 7 \mathrm{~A}$ & $1.354(9)$ & $\mathrm{C} 1 \mathrm{~B}-\mathrm{C} 2 \mathrm{~B}$ & $1.387(10)$ \\
\hline $\mathrm{N} 4 \mathrm{~A}-\mathrm{H} 4 \mathrm{NA}$ & 0.8600 & $\mathrm{C} 1 \mathrm{~B}-\mathrm{C} 6 \mathrm{~B}$ & $1.399(10)$ \\
\hline $\mathrm{N} 5 \mathrm{~A}-\mathrm{C} 13 \mathrm{~A}$ & $1.370(9)$ & $\mathrm{C} 2 \mathrm{~B}-\mathrm{C} 3 \mathrm{~B}$ & $1.350(11)$ \\
\hline $\mathrm{N} 5 \mathrm{~A}-\mathrm{C} 14 \mathrm{~A}$ & $1.372(9)$ & $\mathrm{C} 2 \mathrm{~B}-\mathrm{H} 2 \mathrm{BA}$ & 0.9300 \\
\hline N5A-H5NA & 0.8600 & $\mathrm{C} 3 \mathrm{~B}-\mathrm{C} 4 \mathrm{~B}$ & $1.394(12)$ \\
\hline $\mathrm{C} 1 \mathrm{~A}-\mathrm{C} 6 \mathrm{~A}$ & $1.400(10)$ & $\mathrm{C} 3 \mathrm{~B}-\mathrm{H} 3 \mathrm{BA}$ & 0.9300 \\
\hline $\mathrm{C} 1 \mathrm{~A}-\mathrm{C} 2 \mathrm{~A}$ & $1.397(11)$ & $\mathrm{C} 4 \mathrm{~B}-\mathrm{C} 5 \mathrm{~B}$ & $1.432(13)$ \\
\hline $\mathrm{C} 2 \mathrm{~A}-\mathrm{C} 3 \mathrm{~A}$ & $1.350(11)$ & $\mathrm{C} 4 \mathrm{~B}-\mathrm{H} 4 \mathrm{BA}$ & 0.9300 \\
\hline $\mathrm{C} 2 \mathrm{~A}-\mathrm{H} 2 \mathrm{AA}$ & 0.9300 & $\mathrm{C} 5 \mathrm{~B}-\mathrm{C} 6 \mathrm{~B}$ & $1.389(11)$ \\
\hline $\mathrm{C} 3 \mathrm{~A}-\mathrm{C} 4 \mathrm{~A}$ & $1.395(12)$ & $\mathrm{C} 5 \mathrm{~B}-\mathrm{H} 5 \mathrm{BA}$ & 0.9300 \\
\hline $\mathrm{C} 3 \mathrm{~A}-\mathrm{H} 3 \mathrm{AA}$ & 0.9300 & $\mathrm{C} 7 \mathrm{~B}-\mathrm{C} 8 \mathrm{~B}$ & $1.449(10)$ \\
\hline $\mathrm{C} 4 \mathrm{~A}-\mathrm{C} 5 \mathrm{~A}$ & $1.438(13)$ & $\mathrm{C} 8 \mathrm{~B}-\mathrm{C} 9 \mathrm{~B}$ & $1.394(10)$ \\
\hline $\mathrm{C} 4 \mathrm{~A}-\mathrm{H} 4 \mathrm{AA}$ & 0.9300 & $\mathrm{C} 9 \mathrm{~B}-\mathrm{C} 10 \mathrm{~B}$ & $1.365(11)$ \\
\hline $\mathrm{C} 5 \mathrm{~A}-\mathrm{C} 6 \mathrm{~A}$ & $1.395(11)$ & $\mathrm{C} 9 \mathrm{~B}-\mathrm{H} 9 \mathrm{BA}$ & 0.9300 \\
\hline $\mathrm{C} 5 \mathrm{~A}-\mathrm{H} 5 \mathrm{AA}$ & 0.9300 & $\mathrm{C} 10 \mathrm{~B}-\mathrm{C} 11 \mathrm{~B}$ & $1.429(11)$ \\
\hline $\mathrm{C} 7 \mathrm{~A}-\mathrm{C} 8 \mathrm{~A}$ & $1.453(10)$ & $\mathrm{C} 10 \mathrm{~B}-\mathrm{H} 10 \mathrm{~B}$ & 0.9300 \\
\hline $\mathrm{C} 8 \mathrm{~A}-\mathrm{C} 9 \mathrm{~A}$ & $1.390(10)$ & $\mathrm{C} 11 \mathrm{~B}-\mathrm{C} 12 \mathrm{~B}$ & $1.375(10)$ \\
\hline $\mathrm{C} 9 \mathrm{~A}-\mathrm{C} 10 \mathrm{~A}$ & $1.365(11)$ & $\mathrm{C} 11 \mathrm{~B}-\mathrm{H} 11 \mathrm{~B}$ & 0.9300 \\
\hline C9A-H9AA & 0.9300 & $\mathrm{C} 12 \mathrm{~B}-\mathrm{C} 13 \mathrm{~B}$ & $1.417(9)$ \\
\hline $\mathrm{C} 10 \mathrm{~A}-\mathrm{C} 11 \mathrm{~A}$ & $1.427(11)$ & $\mathrm{C} 14 \mathrm{~B}-\mathrm{C} 15 \mathrm{~B}$ & $1.359(10)$ \\
\hline $\mathrm{C} 10 \mathrm{~A}-\mathrm{H} 10 \mathrm{~A}$ & 0.9300 & $\mathrm{C} 14 \mathrm{~B}-\mathrm{C} 19 \mathrm{~B}$ & $1.388(10)$ \\
\hline $\mathrm{C} 11 \mathrm{~A}-\mathrm{C} 12 \mathrm{~A}$ & $1.377(9)$ & $\mathrm{C} 15 \mathrm{~B}-\mathrm{C} 16 \mathrm{~B}$ & $1.354(11)$ \\
\hline $\mathrm{C} 11 \mathrm{~A}-\mathrm{H} 11 \mathrm{~A}$ & 0.9300 & $\mathrm{C} 15 \mathrm{~B}-\mathrm{H} 15 \mathrm{~B}$ & 0.9300 \\
\hline $\mathrm{C} 12 \mathrm{~A}-\mathrm{C} 13 \mathrm{~A}$ & $1.419(9)$ & $\mathrm{C} 16 \mathrm{~B}-\mathrm{C} 17 \mathrm{~B}$ & $1.415(12)$ \\
\hline $\mathrm{C} 14 \mathrm{~A}-\mathrm{C} 15 \mathrm{~A}$ & $1.352(10)$ & $\mathrm{C} 16 \mathrm{~B}-\mathrm{H} 16 \mathrm{~B}$ & 0.9300 \\
\hline $\mathrm{C} 14 \mathrm{~A}-\mathrm{C} 19 \mathrm{~A}$ & $1.385(10)$ & $\mathrm{C} 17 \mathrm{~B}-\mathrm{C} 18 \mathrm{~B}$ & $1.353(11)$ \\
\hline $\mathrm{C} 15 \mathrm{~A}-\mathrm{C} 16 \mathrm{~A}$ & $1.349(11)$ & $\mathrm{C} 17 \mathrm{~B}-\mathrm{H} 17 \mathrm{~B}$ & 0.9300 \\
\hline $\mathrm{C} 15 \mathrm{~A}-\mathrm{H} 15 \mathrm{~A}$ & 0.9300 & $\mathrm{C} 18 \mathrm{~B}-\mathrm{C} 19 \mathrm{~B}$ & $1.401(10)$ \\
\hline $\mathrm{C} 16 \mathrm{~A}-\mathrm{C} 17 \mathrm{~A}$ & $1.414(12)$ & $\mathrm{C} 18 \mathrm{~B}-\mathrm{H} 18 \mathrm{~B}$ & 0.9300 \\
\hline $\mathrm{C} 16 \mathrm{~A}-\mathrm{H} 16 \mathrm{~A}$ & 0.9300 & $\mathrm{~S} 1-\mathrm{O} 3$ & $1.436(7)$ \\
\hline $\mathrm{C} 17 \mathrm{~A}-\mathrm{C} 18 \mathrm{~A}$ & $1.350(11)$ & $\mathrm{S} 1-\mathrm{O} 4$ & $1.464(9)$ \\
\hline $\mathrm{C} 17 \mathrm{~A}-\mathrm{H} 17 \mathrm{~A}$ & 0.9300 & $\mathrm{~S} 1-\mathrm{O} 2$ & $1.469(8)$ \\
\hline $\mathrm{C} 18 \mathrm{~A}-\mathrm{C} 19 \mathrm{~A}$ & $1.403(10)$ & $\mathrm{S} 1-\mathrm{O} 1$ & $1.509(8)$ \\
\hline
\end{tabular}




$\begin{array}{ll}\text { N2B-Ni1-N2A } & 172.9(3) \\ \text { N2B-Ni1-N3B } & 76.7(3) \\ \text { N2A-Ni1-N3B } & 99.1(3) \\ \text { N2B-Ni1-N3A } & 109.4(3) \\ \text { N2A-Ni1-N3A } & 76.1(3) \\ \text { N3B-Ni1-N3A } & 91.8(3) \\ \text { N2B-Ni1-N1A } & 96.1(3) \\ \text { N2A-Ni1-N1A } & 78.3(3) \\ \text { N3B-Ni1-N1A } & 92.5(3) \\ \text { N3A-Ni1-N1A } & 154.4(3) \\ \text { N2B-Ni1-N1B } & 77.7(2) \\ \text { N2A-Ni1-N1B } & 106.7(3) \\ \text { N3B-Ni1-N1B } & 154.2(3) \\ \text { N3A-Ni1-N1B } & 93.8(3) \\ \text { N1A-Ni1-N1B } & 93.2(3) \\ \text { C7A-N1A-C1A } & 105.0(7) \\ \text { C7A-N1A-Ni1 } & 110.3(5) \\ \text { C1A-N1A-Ni1 } & 144.7(6) \\ \text { C12A-N2A-C8A } & 120.4(7) \\ \text { C12A-N2A-Ni1 } & 120.2(5) \\ \text { C8A-N2A-Ni1 } & 119.2(5) \\ \text { C13A-N3A-C19A } & 104.3(6) \\ \text { C13A-N3A-Ni1 } & 112.6(5) \\ \text { C19A-N3A-Ni1 } & 143.1(5) \\ \text { C6A-N4A-C7A } & 107.9(7) \\ \text { C6A-N4A-H4NA } & 126.0 \\ \text { C7A-N4A-H4NA } & 126.0 \\ \text { C13A-N5A-C14A } & 108.7(7) \\ \text { C13A-N5A-H5NA } & 125.6 \\ \text { C14A-N5A-H5NA } & 125.6 \\ \text { N1A-C1A-C6A } & 109.1(7) \\ \text { N1A-C1A-C2A } & 130.5(8) \\ \text { C6A-C1A-C2A } & 120.3(8) \\ \text { C3A-C2A-C1A } & 118.0(9) \\ \text { C3A-C2A-H2AA } & 121.0 \\ \text { C1A-C2A-H2AA } & 121.0 \\ \text { C2A-C3A-C4A } & 120.9(10) \\ \text { C2A-C3A-H3AA } & 119.6 \\ \text { C4A-C3A-H3AA } & 119.6 \\ \text { C3A-C4A-C5A } & 124.0(10) \\ \text { C3A-C4A-H4AA } & 118.0 \\ \text { C5A-C4A-H4AA } & 118.0 \\ \text { C6A-C5A-C4A } & 111.5(9) \\ \text { C6A-C5A-H5AA } & 124.3 \\ \text { C4A-C5A-H5AA } & 124.3 \\ \text { N4A-C6A-C1A } & \text { N4A-C6A-C5A } \\ & \end{array}$

\begin{tabular}{|c|c|}
\hline $\mathrm{C} 19 \mathrm{~A}-\mathrm{C} 18 \mathrm{~A}-\mathrm{H} 18 \mathrm{~A}$ & 121.7 \\
\hline $\mathrm{C} 14 \mathrm{~A}-\mathrm{C} 19 \mathrm{~A}-\mathrm{C} 18 \mathrm{~A}$ & $120.3(8)$ \\
\hline $\mathrm{C} 14 \mathrm{~A}-\mathrm{C} 19 \mathrm{~A}-\mathrm{N} 3 \mathrm{~A}$ & $110.6(7)$ \\
\hline $\mathrm{C} 18 \mathrm{~A}-\mathrm{C} 19 \mathrm{~A}-\mathrm{N} 3 \mathrm{~A}$ & $128.9(8)$ \\
\hline $\mathrm{C} 7 \mathrm{~B}-\mathrm{N} 1 \mathrm{~B}-\mathrm{C} 1 \mathrm{~B}$ & $105.8(7)$ \\
\hline $\mathrm{C} 7 \mathrm{~B}-\mathrm{N} 1 \mathrm{~B}-\mathrm{Ni} 1$ & $110.2(5)$ \\
\hline C1B-N1B-Ni1 & $143.4(5)$ \\
\hline $\mathrm{C} 12 \mathrm{~B}-\mathrm{N} 2 \mathrm{~B}-\mathrm{C} 8 \mathrm{~B}$ & $120.0(7)$ \\
\hline $\mathrm{C} 12 \mathrm{~B}-\mathrm{N} 2 \mathrm{~B}-\mathrm{Ni1}$ & $120.0(5)$ \\
\hline $\mathrm{C} 8 \mathrm{~B}-\mathrm{N} 2 \mathrm{~B}-\mathrm{Ni1}$ & $119.9(5)$ \\
\hline $\mathrm{C} 13 \mathrm{~B}-\mathrm{N} 3 \mathrm{~B}-\mathrm{C} 19 \mathrm{~B}$ & $103.4(6)$ \\
\hline $\mathrm{C} 13 \mathrm{~B}-\mathrm{N} 3 \mathrm{~B}-\mathrm{Ni1}$ & $112.0(5)$ \\
\hline $\mathrm{C} 19 \mathrm{~B}-\mathrm{N} 3 \mathrm{~B}-\mathrm{Ni1}$ & $144.3(5)$ \\
\hline $\mathrm{C} 7 \mathrm{~B}-\mathrm{N} 4 \mathrm{~B}-\mathrm{C} 6 \mathrm{~B}$ & $107.6(7)$ \\
\hline $\mathrm{C} 7 \mathrm{~B}-\mathrm{N} 4 \mathrm{~B}-\mathrm{H} 4 \mathrm{NB}$ & 126.2 \\
\hline $\mathrm{C} 6 \mathrm{~B}-\mathrm{N} 4 \mathrm{~B}-\mathrm{H} 4 \mathrm{NB}$ & 126.2 \\
\hline $\mathrm{C} 13 \mathrm{~B}-\mathrm{N} 5 \mathrm{~B}-\mathrm{C} 14 \mathrm{~B}$ & $108.7(7)$ \\
\hline $\mathrm{C} 13 \mathrm{~B}-\mathrm{N} 5 \mathrm{~B}-\mathrm{H} 5 \mathrm{NB}$ & 125.6 \\
\hline $\mathrm{C} 14 \mathrm{~B}-\mathrm{N} 5 \mathrm{~B}-\mathrm{H} 5 \mathrm{NB}$ & 125.6 \\
\hline $\mathrm{N} 1 \mathrm{~B}-\mathrm{C} 1 \mathrm{~B}-\mathrm{C} 2 \mathrm{~B}$ & $131.1(8)$ \\
\hline $\mathrm{N} 1 \mathrm{~B}-\mathrm{C} 1 \mathrm{~B}-\mathrm{C} 6 \mathrm{~B}$ & $108.0(7)$ \\
\hline $\mathrm{C} 2 \mathrm{~B}-\mathrm{C} 1 \mathrm{~B}-\mathrm{C} 6 \mathrm{~B}$ & $120.9(8)$ \\
\hline $\mathrm{C} 3 \mathrm{~B}-\mathrm{C} 2 \mathrm{~B}-\mathrm{C} 1 \mathrm{~B}$ & $118.3(9)$ \\
\hline $\mathrm{C} 3 \mathrm{~B}-\mathrm{C} 2 \mathrm{~B}-\mathrm{H} 2 \mathrm{BA}$ & 120.8 \\
\hline $\mathrm{C} 1 \mathrm{~B}-\mathrm{C} 2 \mathrm{~B}-\mathrm{H} 2 \mathrm{BA}$ & 120.8 \\
\hline $\mathrm{C} 2 \mathrm{~B}-\mathrm{C} 3 \mathrm{~B}-\mathrm{C} 4 \mathrm{~B}$ & $120.5(10)$ \\
\hline $\mathrm{C} 2 \mathrm{~B}-\mathrm{C} 3 \mathrm{~B}-\mathrm{H} 3 \mathrm{BA}$ & 119.7 \\
\hline $\mathrm{C} 4 \mathrm{~B}-\mathrm{C} 3 \mathrm{~B}-\mathrm{H} 3 \mathrm{BA}$ & 119.7 \\
\hline $\mathrm{C} 3 \mathrm{~B}-\mathrm{C} 4 \mathrm{~B}-\mathrm{C} 5 \mathrm{~B}$ & $124.1(10)$ \\
\hline $\mathrm{C} 3 \mathrm{~B}-\mathrm{C} 4 \mathrm{~B}-\mathrm{H} 4 \mathrm{BA}$ & 118.0 \\
\hline $\mathrm{C} 5 \mathrm{~B}-\mathrm{C} 4 \mathrm{~B}-\mathrm{H} 4 \mathrm{BA}$ & 118.0 \\
\hline $\mathrm{C} 6 \mathrm{~B}-\mathrm{C} 5 \mathrm{~B}-\mathrm{C} 4 \mathrm{~B}$ & $112.5(9)$ \\
\hline $\mathrm{C} 6 \mathrm{~B}-\mathrm{C} 5 \mathrm{~B}-\mathrm{H} 5 \mathrm{BA}$ & 123.7 \\
\hline $\mathrm{C} 4 \mathrm{~B}-\mathrm{C} 5 \mathrm{~B}-\mathrm{H} 5 \mathrm{BA}$ & 123.7 \\
\hline $\mathrm{N} 4 \mathrm{~B}-\mathrm{C} 6 \mathrm{~B}-\mathrm{C} 5 \mathrm{~B}$ & $129.0(8)$ \\
\hline $\mathrm{N} 4 \mathrm{~B}-\mathrm{C} 6 \mathrm{~B}-\mathrm{C} 1 \mathrm{~B}$ & $107.4(8)$ \\
\hline $\mathrm{C} 5 \mathrm{~B}-\mathrm{C} 6 \mathrm{~B}-\mathrm{C} 1 \mathrm{~B}$ & $123.5(9)$ \\
\hline $\mathrm{N} 4 \mathrm{~B}-\mathrm{C} 7 \mathrm{~B}-\mathrm{N} 1 \mathrm{~B}$ & $111.2(7)$ \\
\hline $\mathrm{N} 4 \mathrm{~B}-\mathrm{C} 7 \mathrm{~B}-\mathrm{C} 8 \mathrm{~B}$ & $128.4(7)$ \\
\hline $\mathrm{N} 1 \mathrm{~B}-\mathrm{C} 7 \mathrm{~B}-\mathrm{C} 8 \mathrm{~B}$ & $120.5(7)$ \\
\hline $\mathrm{N} 2 \mathrm{~B}-\mathrm{C} 8 \mathrm{~B}-\mathrm{C} 9 \mathrm{~B}$ & $122.8(8)$ \\
\hline $\mathrm{N} 2 \mathrm{~B}-\mathrm{C} 8 \mathrm{~B}-\mathrm{C} 7 \mathrm{~B}$ & $111.1(7)$ \\
\hline $\mathrm{C} 9 \mathrm{~B}-\mathrm{C} 8 \mathrm{~B}-\mathrm{C} 7 \mathrm{~B}$ & $126.0(8)$ \\
\hline $\mathrm{C} 10 \mathrm{~B}-\mathrm{C} 9 \mathrm{~B}-\mathrm{C} 8 \mathrm{~B}$ & $115.8(9)$ \\
\hline $\mathrm{C} 10 \mathrm{~B}-\mathrm{C} 9 \mathrm{~B}-\mathrm{H} 9 \mathrm{BA}$ & 122.1 \\
\hline $\mathrm{C} 8 \mathrm{~B}-\mathrm{C} 9 \mathrm{~B}-\mathrm{H} 9 \mathrm{BA}$ & 122.1 \\
\hline $\mathrm{C} 9 \mathrm{~B}-\mathrm{C} 10 \mathrm{~B}-\mathrm{C} 11 \mathrm{~B}$ & $122.8(9)$ \\
\hline
\end{tabular}




\begin{tabular}{|c|c|}
\hline $\mathrm{C} 1 \mathrm{~A}-\mathrm{C} 6 \mathrm{~A}-\mathrm{C} 5 \mathrm{~A}$ & $124.6(9)$ \\
\hline $\mathrm{N} 1 \mathrm{~A}-\mathrm{C} 7 \mathrm{~A}-\mathrm{N} 4 \mathrm{~A}$ & $111.4(7)$ \\
\hline $\mathrm{N} 1 \mathrm{~A}-\mathrm{C} 7 \mathrm{~A}-\mathrm{C} 8 \mathrm{~A}$ & $121.3(7)$ \\
\hline $\mathrm{N} 4 \mathrm{~A}-\mathrm{C} 7 \mathrm{~A}-\mathrm{C} 8 \mathrm{~A}$ & $127.3(7)$ \\
\hline $\mathrm{N} 2 \mathrm{~A}-\mathrm{C} 8 \mathrm{~A}-\mathrm{C} 9 \mathrm{~A}$ & $123.0(8)$ \\
\hline $\mathrm{N} 2 \mathrm{~A}-\mathrm{C} 8 \mathrm{~A}-\mathrm{C} 7 \mathrm{~A}$ & $110.7(7)$ \\
\hline $\mathrm{C} 9 \mathrm{~A}-\mathrm{C} 8 \mathrm{~A}-\mathrm{C} 7 \mathrm{~A}$ & $126.2(8)$ \\
\hline $\mathrm{C} 10 \mathrm{~A}-\mathrm{C} 9 \mathrm{~A}-\mathrm{C} 8 \mathrm{~A}$ & $116.1(8)$ \\
\hline $\mathrm{C} 10 \mathrm{~A}-\mathrm{C} 9 \mathrm{~A}-\mathrm{H} 9 \mathrm{AA}$ & 121.9 \\
\hline C8A-C9A-H9AA & 121.9 \\
\hline $\mathrm{C} 9 \mathrm{~A}-\mathrm{C} 10 \mathrm{~A}-\mathrm{C} 11 \mathrm{~A}$ & $121.8(9)$ \\
\hline $\mathrm{C} 9 \mathrm{~A}-\mathrm{C} 10 \mathrm{~A}-\mathrm{H} 10 \mathrm{~A}$ & 119.1 \\
\hline $\mathrm{C} 11 \mathrm{~A}-\mathrm{C} 10 \mathrm{~A}-\mathrm{H} 10 \mathrm{~A}$ & 119.1 \\
\hline $\mathrm{C} 12 \mathrm{~A}-\mathrm{C} 11 \mathrm{~A}-\mathrm{C} 10 \mathrm{~A}$ & $116.8(8)$ \\
\hline $\mathrm{C} 12 \mathrm{~A}-\mathrm{C} 11 \mathrm{~A}-\mathrm{H} 11 \mathrm{~A}$ & 121.6 \\
\hline $\mathrm{C} 10 \mathrm{~A}-\mathrm{C} 11 \mathrm{~A}-\mathrm{H} 11 \mathrm{~A}$ & 121.6 \\
\hline $\mathrm{N} 2 \mathrm{~A}-\mathrm{C} 12 \mathrm{~A}-\mathrm{C} 11 \mathrm{~A}$ & $121.7(8)$ \\
\hline $\mathrm{N} 2 \mathrm{~A}-\mathrm{C} 12 \mathrm{~A}-\mathrm{C} 13 \mathrm{~A}$ & $111.2(7)$ \\
\hline $\mathrm{C} 11 \mathrm{~A}-\mathrm{C} 12 \mathrm{~A}-\mathrm{C} 13 \mathrm{~A}$ & $127.0(8)$ \\
\hline $\mathrm{N} 3 \mathrm{~A}-\mathrm{C} 13 \mathrm{~A}-\mathrm{N} 5 \mathrm{~A}$ & $111.5(7)$ \\
\hline $\mathrm{N} 3 \mathrm{~A}-\mathrm{C} 13 \mathrm{~A}-\mathrm{C} 12 \mathrm{~A}$ & $119.8(7)$ \\
\hline $\mathrm{N} 5 \mathrm{~A}-\mathrm{C} 13 \mathrm{~A}-\mathrm{C} 12 \mathrm{~A}$ & $128.6(7)$ \\
\hline $\mathrm{C} 15 \mathrm{~A}-\mathrm{C} 14 \mathrm{~A}-\mathrm{N} 5 \mathrm{~A}$ & $131.4(8)$ \\
\hline $\mathrm{C} 15 \mathrm{~A}-\mathrm{C} 14 \mathrm{~A}-\mathrm{C} 19 \mathrm{~A}$ & $123.6(9)$ \\
\hline $\mathrm{N} 5 \mathrm{~A}-\mathrm{C} 14 \mathrm{~A}-\mathrm{C} 19 \mathrm{~A}$ & $104.8(7)$ \\
\hline $\mathrm{C} 14 \mathrm{~A}-\mathrm{C} 15 \mathrm{~A}-\mathrm{C} 16 \mathrm{~A}$ & $115.5(9)$ \\
\hline $\mathrm{C} 14 \mathrm{~A}-\mathrm{C} 15 \mathrm{~A}-\mathrm{H} 15 \mathrm{~A}$ & 122.2 \\
\hline $\mathrm{C} 16 \mathrm{~A}-\mathrm{C} 15 \mathrm{~A}-\mathrm{H} 15 \mathrm{~A}$ & 122.2 \\
\hline $\mathrm{C} 15 \mathrm{~A}-\mathrm{C} 16 \mathrm{~A}-\mathrm{C} 17 \mathrm{~A}$ & $123.1(10)$ \\
\hline $\mathrm{C} 15 \mathrm{~A}-\mathrm{C} 16 \mathrm{~A}-\mathrm{H} 16 \mathrm{~A}$ & 118.5 \\
\hline $\mathrm{C} 17 \mathrm{~A}-\mathrm{C} 16 \mathrm{~A}-\mathrm{H} 16 \mathrm{~A}$ & 118.5 \\
\hline $\mathrm{C} 18 \mathrm{~A}-\mathrm{C} 17 \mathrm{~A}-\mathrm{C} 16 \mathrm{~A}$ & $120.9(9)$ \\
\hline $\mathrm{C} 18 \mathrm{~A}-\mathrm{C} 17 \mathrm{~A}-\mathrm{H} 17 \mathrm{~A}$ & 119.6 \\
\hline $\mathrm{C} 16 \mathrm{~A}-\mathrm{C} 17 \mathrm{~A}-\mathrm{H} 17 \mathrm{~A}$ & 119.6 \\
\hline $\mathrm{C} 17 \mathrm{~A}-\mathrm{C} 18 \mathrm{~A}-\mathrm{C} 19 \mathrm{~A}$ & $116.5(9)$ \\
\hline $\mathrm{C} 17 \mathrm{~A}-\mathrm{C} 18 \mathrm{~A}-\mathrm{H} 18 \mathrm{~A}$ & 121.7 \\
\hline
\end{tabular}

\begin{tabular}{|c|c|}
\hline $\mathrm{C} 9 \mathrm{~B}-\mathrm{C} 10 \mathrm{~B}-\mathrm{H} 10 \mathrm{~B}$ & 118.6 \\
\hline $\mathrm{C} 11 \mathrm{~B}-\mathrm{C} 10 \mathrm{~B}-\mathrm{H} 10 \mathrm{~B}$ & 118.6 \\
\hline $\mathrm{C} 12 \mathrm{~B}-\mathrm{C} 11 \mathrm{~B}-\mathrm{C} 10 \mathrm{~B}$ & $115.5(8)$ \\
\hline $\mathrm{C} 12 \mathrm{~B}-\mathrm{C} 11 \mathrm{~B}-\mathrm{H} 11 \mathrm{~B}$ & 122.2 \\
\hline $\mathrm{C} 10 \mathrm{~B}-\mathrm{C} 11 \mathrm{~B}-\mathrm{H} 11 \mathrm{~B}$ & 122.2 \\
\hline $\mathrm{N} 2 \mathrm{~B}-\mathrm{C} 12 \mathrm{~B}-\mathrm{C} 11 \mathrm{~B}$ & $123.0(8)$ \\
\hline $\mathrm{N} 2 \mathrm{~B}-\mathrm{C} 12 \mathrm{~B}-\mathrm{C} 13 \mathrm{~B}$ & $111.2(7)$ \\
\hline $\mathrm{C} 11 \mathrm{~B}-\mathrm{C} 12 \mathrm{~B}-\mathrm{C} 13 \mathrm{~B}$ & $125.8(8)$ \\
\hline $\mathrm{N} 3 \mathrm{~B}-\mathrm{C} 13 \mathrm{~B}-\mathrm{N} 5 \mathrm{~B}$ & $112.2(7)$ \\
\hline $\mathrm{N} 3 \mathrm{~B}-\mathrm{C} 13 \mathrm{~B}-\mathrm{C} 12 \mathrm{~B}$ & $120.1(7)$ \\
\hline $\mathrm{N} 5 \mathrm{~B}-\mathrm{C} 13 \mathrm{~B}-\mathrm{C} 12 \mathrm{~B}$ & $127.7(7)$ \\
\hline $\mathrm{C} 15 \mathrm{~B}-\mathrm{C} 14 \mathrm{~B}-\mathrm{N} 5 \mathrm{~B}$ & $131.2(9)$ \\
\hline $\mathrm{C} 15 \mathrm{~B}-\mathrm{C} 14 \mathrm{~B}-\mathrm{C} 19 \mathrm{~B}$ & $123.9(8)$ \\
\hline $\mathrm{N} 5 \mathrm{~B}-\mathrm{C} 14 \mathrm{~B}-\mathrm{C} 19 \mathrm{~B}$ & $104.6(7)$ \\
\hline $\mathrm{C} 16 \mathrm{~B}-\mathrm{C} 15 \mathrm{~B}-\mathrm{C} 14 \mathrm{~B}$ & $114.7(9)$ \\
\hline $\mathrm{C} 16 \mathrm{~B}-\mathrm{C} 15 \mathrm{~B}-\mathrm{H} 15 \mathrm{~B}$ & 122.7 \\
\hline $\mathrm{C} 14 \mathrm{~B}-\mathrm{C} 15 \mathrm{~B}-\mathrm{H} 15 \mathrm{~B}$ & 122.7 \\
\hline $\mathrm{C} 15 \mathrm{~B}-\mathrm{C} 16 \mathrm{~B}-\mathrm{C} 17 \mathrm{~B}$ & $123.6(10)$ \\
\hline $\mathrm{C} 15 \mathrm{~B}-\mathrm{C} 16 \mathrm{~B}-\mathrm{H} 16 \mathrm{~B}$ & 118.2 \\
\hline $\mathrm{C} 17 \mathrm{~B}-\mathrm{C} 16 \mathrm{~B}-\mathrm{H} 16 \mathrm{~B}$ & 118.2 \\
\hline $\mathrm{C} 18 \mathrm{~B}-\mathrm{C} 17 \mathrm{~B}-\mathrm{C} 16 \mathrm{~B}$ & $120.8(9)$ \\
\hline $\mathrm{C} 18 \mathrm{~B}-\mathrm{C} 17 \mathrm{~B}-\mathrm{H} 17 \mathrm{~B}$ & 119.6 \\
\hline $\mathrm{C} 16 \mathrm{~B}-\mathrm{C} 17 \mathrm{~B}-\mathrm{H} 17 \mathrm{~B}$ & 119.6 \\
\hline $\mathrm{C} 17 \mathrm{~B}-\mathrm{C} 18 \mathrm{~B}-\mathrm{C} 19 \mathrm{~B}$ & $116.4(9)$ \\
\hline $\mathrm{C} 17 \mathrm{~B}-\mathrm{C} 18 \mathrm{~B}-\mathrm{H} 18 \mathrm{~B}$ & 121.8 \\
\hline $\mathrm{C} 19 \mathrm{~B}-\mathrm{C} 18 \mathrm{~B}-\mathrm{H} 18 \mathrm{~B}$ & 121.8 \\
\hline $\mathrm{C} 14 \mathrm{~B}-\mathrm{C} 19 \mathrm{~B}-\mathrm{C} 18 \mathrm{~B}$ & $120.4(8)$ \\
\hline $\mathrm{C} 14 \mathrm{~B}-\mathrm{C} 19 \mathrm{~B}-\mathrm{N} 3 \mathrm{~B}$ & $110.8(7)$ \\
\hline $\mathrm{C} 18 \mathrm{~B}-\mathrm{C} 19 \mathrm{~B}-\mathrm{N} 3 \mathrm{~B}$ & $128.6(8)$ \\
\hline $\mathrm{O} 3-\mathrm{S} 1-\mathrm{O} 4$ & $107.4(5)$ \\
\hline $\mathrm{O} 3-\mathrm{S} 1-\mathrm{O} 2$ & $112.8(5)$ \\
\hline $\mathrm{O} 4-\mathrm{S} 1-\mathrm{O} 2$ & $111.5(5)$ \\
\hline $\mathrm{O} 3-\mathrm{S} 1-\mathrm{O} 1$ & $111.0(6)$ \\
\hline $\mathrm{O} 4-\mathrm{S} 1-\mathrm{O} 1$ & $108.4(5)$ \\
\hline $\mathrm{O} 2-\mathrm{S} 1-\mathrm{O} 1$ & $105.7(5)$ \\
\hline
\end{tabular}

Hydrogen-bond geometry $\left(A,{ }^{\circ}\right)$

\begin{tabular}{lllll}
\hline$D-\mathrm{H} \cdots A$ & $D-\mathrm{H}$ & $\mathrm{H} \cdots A$ & $D \cdots A$ & $D-\mathrm{H} \cdots A$ \\
\hline $\mathrm{N} 4 A-\mathrm{H} 4 N A \cdots \mathrm{O} 3^{\mathrm{i}}$ & 0.86 & 1.85 & $2.706(10)$ & 172 \\
$\mathrm{~N} 5 A-\mathrm{H} 5 N A \cdots \mathrm{O} 2^{\mathrm{ii}}$ & 0.86 & 2.04 & $2.883(11)$ & 165 \\
$\mathrm{~N} 4 B-\mathrm{H} 4 N B \cdots \mathrm{O} 4^{\mathrm{iii}}$ & 0.86 & 1.89 & $2.746(10)$ & 177 \\
$\mathrm{~N} 5 B-\mathrm{H} 5 N B \cdots \mathrm{O} 1$ & 0.86 & 1.75 & $2.605(10)$ & 174 \\
\hline
\end{tabular}

Symmetry codes: (i) $y,-x+1,-z$; (ii) $-x+3 / 2,-y+1 / 2, z-1 / 2$; (iii) $-x+3 / 2,-y+1 / 2, z+1 / 2$. 
Bis[2,6-bis $\left(1 H\right.$-benzimidazol-2-yl- $\left.\kappa N^{3}\right)$ pyridine- $\left.\kappa N\right]$ nickel(II) sulfate-dimethylformamide-water (1/1/4.25) (III)

Crystal data

$\left[\mathrm{Ni}\left(\mathrm{C}_{19} \mathrm{H}_{13} \mathrm{~N}_{5}\right)_{2}\right] \mathrm{SO}_{4} \cdot \mathrm{C}_{3} \mathrm{H}_{7} \mathrm{NO} \cdot 4.25 \mathrm{H}_{2} \mathrm{O}$

$D_{\mathrm{x}}=1.338 \mathrm{Mg} \mathrm{m}^{-3}$

$M_{r}=925.10$

Orthorhombic, Pccn

$a=13.406(5) \AA$

$b=24.365(5) \AA$

$c=28.114(5) \AA$

$V=9183(4) \AA^{3}$

$Z=8$

$F(000)=3844$

Mo $K \alpha$ radiation, $\lambda=0.71073 \AA$

Cell parameters from 5212 reflections

$\theta=3.6-28.0^{\circ}$

$\mu=0.53 \mathrm{~mm}^{-1}$

$T=294 \mathrm{~K}$

Fragment, light red

$0.28 \times 0.18 \times 0.14 \mathrm{~mm}$

\section{Data collection}

Oxford Diffraction Gemini CCD S Ultra diffractometer

Radiation source: fine-focus sealed tube Graphite monochromator

$\omega$ scans, thick slices

Absorption correction: multi-scan

(CrysAlis PRO; Oxford Diffraction, 2009)

$T_{\min }=0.90, T_{\max }=0.94$

32970 measured reflections

10959 independent reflections

4106 reflections with $I>2 \sigma(I)$

$R_{\text {int }}=0.166$

$\theta_{\max }=29.4^{\circ}, \theta_{\min }=3.7^{\circ}$

$h=-16 \rightarrow 18$

$k=-33 \rightarrow 30$

$l=-37 \rightarrow 35$

Refinement

Refinement on $F^{2}$

Least-squares matrix: full

$R\left[F^{2}>2 \sigma\left(F^{2}\right)\right]=0.086$

$w R\left(F^{2}\right)=0.334$

$S=0.85$

10959 reflections

581 parameters

552 restraints

Hydrogen site location: inferred from neighbouring sites

$\mathrm{H}$-atom parameters constrained

$w=1 /\left[\sigma^{2}\left(F_{\mathrm{o}}^{2}\right)+(0.1868 P)^{2}\right]$

where $P=\left(F_{\mathrm{o}}^{2}+2 F_{\mathrm{c}}^{2}\right) / 3$

$(\Delta / \sigma)_{\max }<0.001$

$\Delta \rho_{\max }=0.67 \mathrm{e} \AA^{-3}$

$\Delta \rho_{\min }=-0.52$ e $\AA^{-3}$

Special details

Geometry. All e.s.d.'s (except the e.s.d. in the dihedral angle between two 1.s. planes) are estimated using the full covariance matrix. The cell e.s.d.'s are taken into account individually in the estimation of e.s.d.'s in distances, angles and torsion angles; correlations between e.s.d.'s in cell parameters are only used when they are defined by crystal symmetry. An approximate (isotropic) treatment of cell e.s.d.'s is used for estimating e.s.d.'s involving l.s. planes.

Fractional atomic coordinates and isotropic or equivalent isotropic displacement parameters $\left(\AA^{2}\right)$

\begin{tabular}{llllll}
\hline & $x$ & $y$ & $z$ & $U_{\text {iso }} * / U_{\text {eq }}$ & Occ. $(<1)$ \\
\hline Ni1 & $0.76257(5)$ & $0.45859(3)$ & $0.36949(3)$ & $0.0429(3)$ & \\
N1A & $0.8559(3)$ & $0.5276(2)$ & $0.35855(18)$ & $0.0468(11)$ & \\
N2A & $0.8718(3)$ & $0.4458(2)$ & $0.41757(17)$ & $0.0435(10)$ & \\
N3A & $0.7174(3)$ & $0.3858(2)$ & $0.40197(18)$ & $0.0497(12)$ & \\
N4A & $0.9952(4)$ & $0.5700(2)$ & $0.38078(18)$ & $0.0505(12)$ & \\
H4AA & 1.049741 & 0.576304 & 0.395923 & $0.061^{*}$ & \\
N5A & $0.7388(3)$ & $0.3281(2)$ & $0.46274(19)$ & $0.0553(13)$ & \\
H5AA & 0.765735 & 0.313306 & 0.487346 & $0.066^{*}$ & \\
C1A & $0.8650(4)$ & $0.5757(3)$ & $0.3333(2)$ & $0.0511(14)$ & \\
C2A & $0.8038(5)$ & $0.5982(3)$ & $0.2980(3)$ & $0.0691(19)$ &
\end{tabular}




\begin{tabular}{|c|c|c|c|c|}
\hline $\mathrm{H} 2 \mathrm{~A}$ & 0.746253 & 0.580038 & 0.288211 & $0.083^{*}$ \\
\hline $\mathrm{C} 3 \mathrm{~A}$ & $0.8301(6)$ & $0.6472(4)$ & $0.2781(3)$ & $0.093(3)$ \\
\hline $\mathrm{H} 3 \mathrm{~A}$ & 0.789639 & 0.663026 & 0.255010 & $0.111 *$ \\
\hline $\mathrm{C} 4 \mathrm{~A}$ & $0.9189(6)$ & $0.6741(3)$ & $0.2926(3)$ & $0.088(2)$ \\
\hline $\mathrm{H} 4 \mathrm{~A}$ & 0.936407 & 0.707106 & 0.278141 & $0.106^{*}$ \\
\hline C5A & 0.9805 (5) & $0.6528(3)$ & 0.3277 (3) & $0.080(2)$ \\
\hline H5A & 1.037865 & 0.670817 & 0.337802 & $0.096^{*}$ \\
\hline C6A & 0.9515 (4) & $0.6033(3)$ & $0.3466(2)$ & $0.0551(14)$ \\
\hline C7A & 0.9359 (4) & $0.5258(3)$ & $0.3857(2)$ & $0.0434(12)$ \\
\hline $\mathrm{C} 8 \mathrm{~A}$ & 0.9517 (4) & $0.4791(2)$ & $0.41858(19)$ & $0.0392(11)$ \\
\hline C9A & $1.0338(4)$ & $0.4684(3)$ & $0.4457(2)$ & 0.0495 (14) \\
\hline H9A & 1.089451 & 0.491149 & 0.444945 & $0.059^{*}$ \\
\hline $\mathrm{C} 10 \mathrm{~A}$ & $1.0310(5)$ & $0.4218(3)$ & $0.4746(2)$ & $0.0566(15)$ \\
\hline H10A & 1.085433 & 0.413438 & 0.493752 & $0.068^{*}$ \\
\hline C11A & $0.9491(4)$ & 0.3883 & $0.4751(2)$ & $0.0534(15)$ \\
\hline H11A & 0.947521 & 0.357633 & 0.494835 & $0.064 *$ \\
\hline C12A & 0.8689 (4) & $0.4002(2)$ & $0.4461(2)$ & $0.0461(12)$ \\
\hline C13A & 0.7750 (4) & $0.3711(3)$ & $0.4375(2)$ & $0.0468(12)$ \\
\hline C14A & 0.6507 (4) & $0.3128(3)$ & $0.4415(2)$ & $0.0568(15)$ \\
\hline $\mathrm{C} 15 \mathrm{~A}$ & $0.5796(5)$ & $0.2716(3)$ & $0.4507(3)$ & $0.079(2)$ \\
\hline $\mathrm{H} 15 \mathrm{~A}$ & 0.586130 & 0.248240 & 0.476645 & $0.095^{*}$ \\
\hline C16A & 0.5005 (6) & $0.2671(4)$ & $0.4201(3)$ & $0.086(2)$ \\
\hline H16A & 0.453090 & 0.239953 & 0.425587 & $0.103^{*}$ \\
\hline C17A & $0.4883(6)$ & $0.3022(3)$ & $0.3808(3)$ & $0.081(2)$ \\
\hline H17A & 0.434175 & 0.297596 & 0.360529 & $0.097 *$ \\
\hline C18A & $0.5560(5)$ & $0.3431(3)$ & $0.3724(3)$ & $0.0628(17)$ \\
\hline H18A & 0.547589 & 0.366991 & 0.346841 & $0.075^{*}$ \\
\hline C19A & 0.6383 (4) & $0.3485(3)$ & $0.4028(2)$ & $0.0521(14)$ \\
\hline N1B & $0.8208(3)$ & $0.4178(2)$ & $0.30917(18)$ & 0.0481 (11) \\
\hline N2B & $0.6529(3)$ & 0.4703 (2) & $0.32124(17)$ & 0.0445 (11) \\
\hline N3B & 0.6567 (3) & $0.5035(2)$ & $0.40849(17)$ & 0.0464 (11) \\
\hline N4B & 0.7954 (4) & $0.3962(2)$ & $0.23290(18)$ & $0.0540(12)$ \\
\hline H4BA & 0.765711 & 0.393393 & 0.205878 & $0.065^{*}$ \\
\hline N5B & $0.5092(4)$ & 0.5443 (2) & $0.40806(19)$ & $0.0553(13)$ \\
\hline H5BA & 0.452919 & 0.556436 & 0.397816 & $0.066^{*}$ \\
\hline C1B & 0.9025 (4) & $0.3893(3)$ & $0.2918(2)$ & $0.0520(14)$ \\
\hline $\mathrm{C} 2 \mathrm{~B}$ & 0.9899 (5) & 0.3719 (3) & $0.3153(3)$ & $0.071(2)$ \\
\hline H2B & 0.999623 & 0.378303 & 0.347550 & $0.085^{*}$ \\
\hline C3B & $1.0604(5)$ & $0.3450(4)$ & $0.2882(3)$ & 0.089 \\
\hline H3B & 1.119765 & 0.334159 & 0.302469 & $0.107 *$ \\
\hline C4B & $1.0455(5)$ & $0.3334(3)$ & 0.2398 & $0.075(2)$ \\
\hline H4B & 1.095094 & 0.315615 & 0.222607 & $0.090^{*}$ \\
\hline C5B & $0.9590(5)$ & $0.3481(3)$ & $0.2179(3)$ & 0.0712 (19) \\
\hline H5B & 0.948269 & 0.339847 & 0.185998 & $0.085^{*}$ \\
\hline C6B & 0.8871 (4) & $0.3757(3)$ & $0.2442(2)$ & 0.0535 (14) \\
\hline C7B & 0.7601 (4) & $0.4215(3)$ & $0.2716(2)$ & 0.0469 (13) \\
\hline C8B & 0.6649 (4) & 0.4508 (3) & $0.2758(2)$ & $0.0472(13)$ \\
\hline C9B & $0.5934(5)$ & $0.4584(3)$ & $0.2424(2)$ & $0.0616(17)$ \\
\hline
\end{tabular}




\begin{tabular}{|c|c|c|c|c|c|}
\hline H9B & 0.602302 & 0.445324 & 0.211601 & $0.074 *$ & \\
\hline C10B & $0.5063(5)$ & $0.4861(3)$ & $0.2549(3)$ & $0.075(2)$ & \\
\hline H10B & 0.455736 & 0.490905 & 0.232645 & $0.090 *$ & \\
\hline C11B & $0.4953(5)$ & 0.5068 & $0.3016(2)$ & $0.0596(16)$ & \\
\hline H11B & 0.438064 & 0.525831 & 0.310438 & $0.072 *$ & \\
\hline C12B & 0.5709 (4) & $0.4982(2)$ & $0.3337(2)$ & $0.0464(13)$ & \\
\hline C13B & $0.5761(4)$ & 0.5152 & $0.3831(2)$ & $0.0463(13)$ & \\
\hline C14B & $0.5470(4)$ & 0.5508 & $0.4526(2)$ & 0.0507 (13) & \\
\hline C15B & $0.5101(5)$ & 0.5775 & 0.4933 & 0.0720 (19) & \\
\hline H15B & 0.449117 & 0.595702 & 0.493034 & $0.086^{*}$ & \\
\hline $\mathrm{C} 16 \mathrm{~B}$ & $0.5678(5)$ & $0.5756(3)$ & 0.5333 & 0.0718 (19) & \\
\hline H16B & 0.544805 & 0.592263 & 0.561015 & $0.086^{*}$ & \\
\hline C17B & $0.6605(5)$ & $0.5494(3)$ & 0.5338 & $0.073(2)$ & \\
\hline H17B & 0.698427 & 0.550032 & 0.561528 & $0.087^{*}$ & \\
\hline C18B & $0.6966(5)$ & $0.5229(3)$ & $0.4948(2)$ & $0.0582(16)$ & \\
\hline H18B & 0.756945 & 0.504184 & 0.495799 & $0.070^{*}$ & \\
\hline C19B & $0.6401(4)$ & $0.5250(3)$ & $0.4536(2)$ & 0.0463 (13) & \\
\hline $\mathrm{O} 1 \mathrm{C}$ & $0.7965(6)$ & $0.6719(3)$ & 0.5069 & $0.141(3)$ & \\
\hline $\mathrm{N} 1 \mathrm{C}$ & $0.6856(6)$ & $0.6834(3)$ & 0.4458 & $0.108(2)$ & \\
\hline $\mathrm{C} 1 \mathrm{C}$ & $0.7529(10)$ & $0.6622(6)$ & $0.4125(5)$ & $0.187(6)$ & \\
\hline $\mathrm{H} 1 \mathrm{C} 1$ & 0.804412 & 0.642226 & 0.428683 & $0.280 *$ & \\
\hline $\mathrm{H} 1 \mathrm{C} 2$ & 0.782140 & 0.691813 & 0.394775 & $0.280 *$ & \\
\hline H1C3 & 0.718286 & 0.638075 & 0.391136 & $0.280 *$ & \\
\hline $\mathrm{C} 2 \mathrm{C}$ & $0.5885(9)$ & $0.7037(6)$ & $0.4343(6)$ & $0.186(6)$ & \\
\hline $\mathrm{H} 2 \mathrm{C} 1$ & 0.559437 & 0.720396 & 0.461972 & $0.279 *$ & \\
\hline $\mathrm{H} 2 \mathrm{C} 2$ & 0.547135 & 0.673844 & 0.423929 & $0.279 *$ & \\
\hline $\mathrm{H} 2 \mathrm{C} 3$ & 0.593710 & 0.730421 & 0.409372 & $0.279 *$ & \\
\hline $\mathrm{C} 3 \mathrm{C}$ & $0.7163(9)$ & $0.6863(5)$ & $0.4926(4)$ & $0.117(3)$ & \\
\hline $\mathrm{H} 3 \mathrm{C}$ & 0.671491 & 0.700508 & 0.514672 & $0.140^{*}$ & \\
\hline $\mathrm{S} 1$ & $0.74643(10)$ & $0.41339(7)$ & $0.61042(6)$ & $0.0524(5)$ & \\
\hline $\mathrm{O} 1$ & 0.6698 (4) & $0.3806(3)$ & $0.5878(2)$ & $0.103(2)$ & \\
\hline $\mathrm{O} 2$ & $0.7929(3)$ & $0.3821(2)$ & $0.64958(16)$ & 0.0685 (13) & \\
\hline $\mathrm{O} 3$ & $0.6975(6)$ & $0.4610(2)$ & $0.6293(2)$ & $0.100(2)$ & \\
\hline $\mathrm{O} 4$ & $0.8231(3)$ & $0.4251(3)$ & $0.57542(17)$ & $0.0831(16)$ & \\
\hline $\mathrm{O} 1 \mathrm{~W}$ & $0.8030(7)$ & $0.6612(3)$ & $0.6051(3)$ & $0.154(3)$ & \\
\hline O2WA & $0.7889(11)$ & $0.5565(6)$ & $0.6535(5)$ & $0.107(5)$ & 0.5 \\
\hline $\mathrm{O} 2 \mathrm{WB}$ & $0.714(2)$ & $0.5724(12)$ & $0.6580(11)$ & $0.107(5)$ & 0.25 \\
\hline O3W & 0.8399 (4) & $0.3018(2)$ & $0.54695(17)$ & 0.0882 (17) & \\
\hline $\mathrm{O} 4 \mathrm{~W}$ & $0.6274(5)$ & $0.2747(3)$ & $0.6159(2)$ & 0.1013 (19) & \\
\hline $\mathrm{O} 5 \mathrm{~W}$ & 0.750000 & 0.750000 & $0.6651(5)$ & $0.146(4)$ & \\
\hline
\end{tabular}

Atomic displacement parameters $\left(\AA^{2}\right)$

\begin{tabular}{lllllll}
\hline & $U^{11}$ & $U^{22}$ & $U^{33}$ & $U^{12}$ & $U^{13}$ & $U^{23}$ \\
\hline Ni1 & $0.0302(4)$ & $0.0524(5)$ & $0.0462(5)$ & $0.0020(3)$ & $-0.0044(3)$ & $0.0016(4)$ \\
N1A & $0.0295(19)$ & $0.055(2)$ & $0.056(3)$ & $0.0014(19)$ & $-0.0023(18)$ & $0.005(2)$ \\
N2A & $0.0279(18)$ & $0.055(2)$ & $0.048(2)$ & $0.0039(17)$ & $-0.003(2)$ & $0.0034(19)$ \\
N3A & $0.039(2)$ & $0.055(3)$ & $0.054(3)$ & $-0.0014(19)$ & $-0.003(2)$ & $0.001(2)$
\end{tabular}




\begin{tabular}{|c|c|c|c|c|c|c|}
\hline N4A & $0.034(2)$ & $0.056(3)$ & $0.061(3)$ & $-0.0064(19)$ & $-0.001(2)$ & $-0.002(2)$ \\
\hline N5A & $0.045(2)$ & $0.057(3)$ & $0.064(3)$ & $-0.001(2)$ & $0.002(2)$ & $0.008(2)$ \\
\hline $\mathrm{C} 1 \mathrm{~A}$ & $0.038(2)$ & $0.056(3)$ & 0.059 (3) & $0.003(2)$ & $0.000(2)$ & $0.008(2)$ \\
\hline $\mathrm{C} 2 \mathrm{~A}$ & $0.052(3)$ & $0.072(4)$ & $0.083(4)$ & 0.003 & -0.013 & $0.023(3)$ \\
\hline $\mathrm{C} 3 \mathrm{~A}$ & $0.058(4)$ & $0.090(4)$ & $0.130(7)$ & $0.005(3)$ & -0.008 (4) & $0.053(5)$ \\
\hline $\mathrm{C} 4 \mathrm{~A}$ & $0.060(4)$ & $0.082(5)$ & $0.123(6)$ & $0.003(3)$ & $0.004(4)$ & $0.044(5)$ \\
\hline $\mathrm{C} 5 \mathrm{~A}$ & $0.054(4)$ & $0.073(4)$ & $0.113(5)$ & $-0.005(3)$ & $0.001(4)$ & 0.029 (4) \\
\hline C6A & $0.041(3)$ & $0.057(3)$ & $0.067(4)$ & $0.000(2)$ & $0.001(2)$ & $0.003(2)$ \\
\hline C7A & 0.029 (2) & $0.050(2)$ & $0.051(3)$ & 0.0007 (19) & $-0.0006(19)$ & $0.000(2)$ \\
\hline C8A & $0.0256(18)$ & $0.050(3)$ & $0.042(3)$ & $0.0070(17)$ & $0.0031(18)$ & $-0.005(2)$ \\
\hline C9A & $0.034(2)$ & $0.057(3)$ & $0.057(3)$ & $0.001(2)$ & $-0.011(2)$ & $-0.003(2)$ \\
\hline $\mathrm{C} 10 \mathrm{~A}$ & $0.046(3)$ & $0.057(3)$ & $0.067(4)$ & $0.004(2)$ & -0.018 & $0.000(3)$ \\
\hline C11A & $0.051(2)$ & $0.052(3)$ & 0.057 (3) & $0.003(2)$ & $-0.013(3)$ & $0.004(3)$ \\
\hline $\mathrm{C} 12 \mathrm{~A}$ & $0.042(2)$ & $0.051(3)$ & 0.045 & $0.003(2)$ & $-0.003(2)$ & $0.000(2)$ \\
\hline $\mathrm{C} 13 \mathrm{~A}$ & $0.041(2)$ & 0.049 (3) & $0.051(3)$ & $0.003(2)$ & $0.001(2)$ & $-0.001(2)$ \\
\hline C14A & $0.044(3)$ & $0.055(3)$ & $0.071(3)$ & $0.003(2)$ & $0.001(2)$ & 0.003 (3) \\
\hline $\mathrm{C} 15 \mathrm{~A}$ & $0.056(3)$ & $0.061(4)$ & $0.120(6)$ & -0.004 & 0.011 & 0.005 \\
\hline $\mathrm{C} 16 \mathrm{~A}$ & 0.059 (4) & $0.074(5)$ & $0.124(6)$ & -0.013 & 0.009 (4) & $0.002(4)$ \\
\hline C17A & $0.052(4)$ & $0.076(4)$ & $0.115(5)$ & -0.009 (3) & $0.004(4)$ & $-0.004(4)$ \\
\hline C18A & $0.045(3)$ & $0.070(4)$ & $0.074(4)$ & -0.003 & -0.004 & $-0.003(3)$ \\
\hline C19A & $0.039(2)$ & $0.052(3)$ & $0.066(3)$ & $0.001(2)$ & $0.002(2)$ & $-0.002(2)$ \\
\hline N1B & $0.037(2)$ & 0.053 & $0.055(2)$ & $0.004(2)$ & $-0.0016(17)$ & $0.001(2)$ \\
\hline $\mathrm{N} 2 \mathrm{~B}$ & 0.0304 (19) & $0.051(3)$ & $0.052(2)$ & $0.0024(18)$ & $-0.0052(19)$ & $0.000(2)$ \\
\hline N3B & 0.0297 (19) & $0.057(3)$ & $0.053(2)$ & $0.003(2)$ & $-0.0032(18)$ & $-0.005(2)$ \\
\hline N4B & $0.043(2)$ & 0.065 & $0.054(2)$ & $0.000(2)$ & $0.001(2)$ & $-0.008(2)$ \\
\hline N5B & $0.035(2)$ & $0.067(3)$ & $0.063(2)$ & $0.010(2)$ & $-0.0027(19)$ & $0.004(2)$ \\
\hline C1B & $0.037(2)$ & 0.059 & 0.061 & $0.001(2)$ & $0.002(2)$ & -0.003 \\
\hline $\mathrm{C} 2 \mathrm{~B}$ & 0.045 & $0.094(5)$ & $0.074(4)$ & 0.018 & $-0.002(3)$ & $-0.004(4)$ \\
\hline C3B & $0.050(4)$ & $0.119(6)$ & 0.099 (4) & $0.024(4)$ & $0.000(3)$ & $-0.023(5)$ \\
\hline C4B & $0.048(3)$ & $0.084(5)$ & $0.093(4)$ & 0.008 & $0.013(3)$ & $-0.013(4)$ \\
\hline C5B & $0.051(3)$ & $0.083(5)$ & $0.079(4)$ & $0.000(3)$ & 0.008 & $-0.019(4)$ \\
\hline C6B & $0.040(2)$ & $0.062(4)$ & 0.059 (3) & -0.005 (2) & $0.001(2)$ & -0.003 \\
\hline C7B & $0.039(2)$ & $0.052(3)$ & $0.050(2)$ & $-0.001(2)$ & $0.0001(18)$ & $-0.001(2)$ \\
\hline $\mathrm{C} 8 \mathrm{~B}$ & $0.038(2)$ & $0.054(3)$ & $0.050(2)$ & $-0.001(2)$ & $-0.0026(19)$ & $0.002(2)$ \\
\hline C9B & $0.060(3)$ & $0.066(4)$ & 0.059 (3) & $0.008(3)$ & $-0.020(3)$ & -0.009 \\
\hline $\mathrm{C} 10 \mathrm{~B}$ & $0.064(4)$ & $0.091(5)$ & $0.071(3)$ & 0.020 & $-0.024(3)$ & $-0.014(4)$ \\
\hline C11B & $0.043(3)$ & $0.068(4)$ & $0.067(3)$ & 0.013 & $-0.016(3)$ & $-0.003(3)$ \\
\hline C12B & $0.028(2)$ & 0.055 & $0.056(2)$ & $0.003(2)$ & $-0.0050(18)$ & $0.005(2)$ \\
\hline C13B & $0.030(2)$ & $0.054(3)$ & $0.054(2)$ & -0.002 & $-0.0022(17)$ & $0.007(2)$ \\
\hline C14B & $0.034(2)$ & 0.059 (4) & $0.059(2)$ & $-0.006(2)$ & $0.005(2)$ & $0.006(2)$ \\
\hline $\mathrm{C} 15 \mathrm{~B}$ & $0.053(4)$ & $0.091(5)$ & 0.072 & -0.003 & $0.014(3)$ & $-0.010(3)$ \\
\hline C16B & $0.071(3)$ & $0.078(5)$ & $0.066(3)$ & $-0.002(3)$ & $0.011(3)$ & -0.011 \\
\hline C17B & $0.068(3)$ & $0.091(5)$ & 0.059 & -0.003 & $-0.004(3)$ & $-0.017(3)$ \\
\hline C18B & 0.049 (3) & $0.070(4)$ & $0.056(3)$ & $-0.006(3)$ & $-0.006(2)$ & -0.005 \\
\hline C19B & $0.034(2)$ & $0.053(3)$ & $0.052(2)$ & $-0.006(2)$ & 0.0035 (19) & $-0.003(2)$ \\
\hline $\mathrm{O} 1 \mathrm{C}$ & $0.110(5)$ & $0.125(6)$ & $0.187(7)$ & $0.006(5)$ & $-0.010(5)$ & $0.031(6)$ \\
\hline $\mathrm{N} 1 \mathrm{C}$ & $0.110(5)$ & $0.080(5)$ & $0.133(5)$ & $0.012(4)$ & $0.010(4)$ & $0.013(5)$ \\
\hline $\mathrm{C} 1 \mathrm{C}$ & $0.209(13)$ & $0.138(11)$ & $0.212(12)$ & $0.006(9)$ & $0.087(10)$ & $-0.030(10)$ \\
\hline
\end{tabular}




$\begin{array}{lllllll}\mathrm{C} 2 \mathrm{C} & 0.119(6) & 0.173(12) & 0.266(16) & 0.019(8) & -0.025(8) & 0.058(12) \\ \mathrm{C} 3 \mathrm{C} & 0.103(5) & 0.114(8) & 0.133(6) & 0.008(6) & 0.011(5) & 0.020(6) \\ \mathrm{S} 1 & 0.0344(8) & 0.0605(11) & 0.0623(10) & 0.0042(8) & 0.0034(7) & 0.0013(8) \\ \mathrm{O} 1 & 0.068(3) & 0.110(4) & 0.131(5) & -0.035(3) & -0.039(3) & 0.013(4) \\ \mathrm{O} 2 & 0.051(2) & 0.094(4) & 0.061(3) & 0.022(3) & 0.008(2) & 0.008(3) \\ \mathrm{O} 3 & 0.130(5) & 0.083(4) & 0.089(4) & 0.051(4) & 0.016(4) & -0.009(3) \\ \text { O4 } & 0.041(2) & 0.146(5) & 0.063(3) & -0.012(3) & 0.002(2) & 0.012(3) \\ \text { O1W } & 0.184(7) & 0.103(5) & 0.177(7) & -0.026(6) & -0.060(7) & -0.012(5) \\ \text { O2WA } & 0.138(12) & 0.085(8) & 0.099(7) & -0.043(10) & -0.044(11) & 0.010(7) \\ \text { O2WB } & 0.138(12) & 0.085(8) & 0.099(7) & -0.043(10) & -0.044(11) & 0.010(7) \\ \text { O3W } & 0.131(5) & 0.075(3) & 0.059(3) & 0.017(3) & 0.017(3) & 0.002(3) \\ \text { O4W } & 0.104(4) & 0.087(4) & 0.113(4) & -0.012(4) & 0.033(4) & -0.021(4) \\ \text { O5W } & 0.107(7) & 0.161(10) & 0.170(11) & 0.013(7) & 0.000 & 0.000\end{array}$

Geometric parameters $\left(A,{ }^{\circ}\right)$

\begin{tabular}{|c|c|c|c|}
\hline $\mathrm{Ni1}-\mathrm{N} 2 \mathrm{~A}$ & $2.017(4)$ & $\mathrm{N} 3 \mathrm{~B}-\mathrm{C} 19 \mathrm{~B}$ & $1.389(7)$ \\
\hline Ni1-N2B & $2.021(4)$ & $\mathrm{N} 4 \mathrm{~B}-\mathrm{C} 7 \mathrm{~B}$ & $1.337(7)$ \\
\hline $\mathrm{Ni} 1-\mathrm{N} 3 \mathrm{~A}$ & $2.086(5)$ & $\mathrm{N} 4 \mathrm{~B}-\mathrm{C} 6 \mathrm{~B}$ & $1.365(7)$ \\
\hline Ni1-N3B & $2.101(5)$ & $\mathrm{N} 4 \mathrm{~B}-\mathrm{H} 4 \mathrm{BA}$ & 0.8600 \\
\hline Ni1-N1B & $2.116(5)$ & $\mathrm{N} 5 \mathrm{~B}-\mathrm{C} 13 \mathrm{~B}$ & $1.341(7)$ \\
\hline $\mathrm{Ni1}-\mathrm{N} 1 \mathrm{~A}$ & $2.118(5)$ & $\mathrm{N} 5 \mathrm{~B}-\mathrm{C} 14 \mathrm{~B}$ & $1.360(8)$ \\
\hline $\mathrm{N} 1 \mathrm{~A}-\mathrm{C} 7 \mathrm{~A}$ & $1.316(7)$ & $\mathrm{N} 5 \mathrm{~B}-\mathrm{H} 5 \mathrm{BA}$ & 0.8600 \\
\hline $\mathrm{N} 1 \mathrm{~A}-\mathrm{C} 1 \mathrm{~A}$ & $1.376(8)$ & $\mathrm{C} 1 \mathrm{~B}-\mathrm{C} 6 \mathrm{~B}$ & $1.395(9)$ \\
\hline $\mathrm{N} 2 \mathrm{~A}-\mathrm{C} 8 \mathrm{~A}$ & $1.344(7)$ & $\mathrm{C} 1 \mathrm{~B}-\mathrm{C} 2 \mathrm{~B}$ & $1.410(8)$ \\
\hline $\mathrm{N} 2 \mathrm{~A}-\mathrm{C} 12 \mathrm{~A}$ & $1.372(7)$ & $\mathrm{C} 2 \mathrm{~B}-\mathrm{C} 3 \mathrm{~B}$ & $1.379(9)$ \\
\hline $\mathrm{N} 3 \mathrm{~A}-\mathrm{C} 13 \mathrm{~A}$ & $1.311(7)$ & $\mathrm{C} 2 \mathrm{~B}-\mathrm{H} 2 \mathrm{~B}$ & 0.9300 \\
\hline $\mathrm{N} 3 \mathrm{~A}-\mathrm{C} 19 \mathrm{~A}$ & $1.396(7)$ & $\mathrm{C} 3 \mathrm{~B}-\mathrm{C} 4 \mathrm{~B}$ & $1.403(11)$ \\
\hline $\mathrm{N} 4 \mathrm{~A}-\mathrm{C} 7 \mathrm{~A}$ & $1.345(7)$ & $\mathrm{C} 3 \mathrm{~B}-\mathrm{H} 3 \mathrm{~B}$ & 0.9300 \\
\hline $\mathrm{N} 4 \mathrm{~A}-\mathrm{C} 6 \mathrm{~A}$ & $1.388(8)$ & $\mathrm{C} 4 \mathrm{~B}-\mathrm{C} 5 \mathrm{~B}$ & $1.361(10)$ \\
\hline $\mathrm{N} 4 \mathrm{~A}-\mathrm{H} 4 \mathrm{AA}$ & 0.8600 & $\mathrm{C} 4 \mathrm{~B}-\mathrm{H} 4 \mathrm{~B}$ & 0.9300 \\
\hline $\mathrm{N} 5 \mathrm{~A}-\mathrm{C} 13 \mathrm{~A}$ & $1.356(8)$ & $\mathrm{C} 5 \mathrm{~B}-\mathrm{C} 6 \mathrm{~B}$ & $1.388(9)$ \\
\hline $\mathrm{N} 5 \mathrm{~A}-\mathrm{C} 14 \mathrm{~A}$ & $1.375(7)$ & $\mathrm{C} 5 \mathrm{~B}-\mathrm{H} 5 \mathrm{~B}$ & 0.9300 \\
\hline N5A-H5AA & 0.8600 & $\mathrm{C} 7 \mathrm{~B}-\mathrm{C} 8 \mathrm{~B}$ & $1.468(8)$ \\
\hline $\mathrm{C} 1 \mathrm{~A}-\mathrm{C} 6 \mathrm{~A}$ & $1.392(8)$ & $\mathrm{C} 8 \mathrm{~B}-\mathrm{C} 9 \mathrm{~B}$ & $1.355(8)$ \\
\hline $\mathrm{C} 1 \mathrm{~A}-\mathrm{C} 2 \mathrm{~A}$ & $1.400(9)$ & $\mathrm{C} 9 \mathrm{~B}-\mathrm{C} 10 \mathrm{~B}$ & $1.393(10)$ \\
\hline $\mathrm{C} 2 \mathrm{~A}-\mathrm{C} 3 \mathrm{~A}$ & $1.365(10)$ & C9B-H9B & 0.9300 \\
\hline $\mathrm{C} 2 \mathrm{~A}-\mathrm{H} 2 \mathrm{~A}$ & 0.9300 & $\mathrm{C} 10 \mathrm{~B}-\mathrm{C} 11 \mathrm{~B}$ & $1.416(9)$ \\
\hline $\mathrm{C} 3 \mathrm{~A}-\mathrm{C} 4 \mathrm{~A}$ & $1.420(11)$ & $\mathrm{C} 10 \mathrm{~B}-\mathrm{H} 10 \mathrm{~B}$ & 0.9300 \\
\hline $\mathrm{C} 3 \mathrm{~A}-\mathrm{H} 3 \mathrm{~A}$ & 0.9300 & $\mathrm{C} 11 \mathrm{~B}-\mathrm{C} 12 \mathrm{~B}$ & $1.372(8)$ \\
\hline $\mathrm{C} 4 \mathrm{~A}-\mathrm{C} 5 \mathrm{~A}$ & $1.388(10)$ & $\mathrm{C} 11 \mathrm{~B}-\mathrm{H} 11 \mathrm{~B}$ & 0.9300 \\
\hline $\mathrm{C} 4 \mathrm{~A}-\mathrm{H} 4 \mathrm{~A}$ & 0.9300 & $\mathrm{C} 12 \mathrm{~B}-\mathrm{C} 13 \mathrm{~B}$ & $1.453(8)$ \\
\hline $\mathrm{C} 5 \mathrm{~A}-\mathrm{C} 6 \mathrm{~A}$ & $1.373(9)$ & $\mathrm{C} 14 \mathrm{~B}-\mathrm{C} 19 \mathrm{~B}$ & $1.399(8)$ \\
\hline $\mathrm{C} 5 \mathrm{~A}-\mathrm{H} 5 \mathrm{~A}$ & 0.9300 & $\mathrm{C} 14 \mathrm{~B}-\mathrm{C} 15 \mathrm{~B}$ & $1.407(9)$ \\
\hline $\mathrm{C} 7 \mathrm{~A}-\mathrm{C} 8 \mathrm{~A}$ & $1.482(8)$ & $\mathrm{C} 15 \mathrm{~B}-\mathrm{C} 16 \mathrm{~B}$ & $1.366(9)$ \\
\hline $\mathrm{C} 8 \mathrm{~A}-\mathrm{C} 9 \mathrm{~A}$ & $1.364(7)$ & $\mathrm{C} 15 \mathrm{~B}-\mathrm{H} 15 \mathrm{~B}$ & 0.9300 \\
\hline $\mathrm{C} 9 \mathrm{~A}-\mathrm{C} 10 \mathrm{~A}$ & $1.396(9)$ & $\mathrm{C} 16 \mathrm{~B}-\mathrm{C} 17 \mathrm{~B}$ & $1.397(10)$ \\
\hline C9A-H9A & 0.9300 & $\mathrm{C} 16 \mathrm{~B}-\mathrm{H} 16 \mathrm{~B}$ & 0.9300 \\
\hline
\end{tabular}




\begin{tabular}{|c|c|c|c|}
\hline $\mathrm{C} 10 \mathrm{~A}-\mathrm{C} 11 \mathrm{~A}$ & $1.368(8)$ & $\mathrm{C} 17 \mathrm{~B}-\mathrm{C} 18 \mathrm{~B}$ & $1.362(9)$ \\
\hline $\mathrm{C} 10 \mathrm{~A}-\mathrm{H} 10 \mathrm{~A}$ & 0.9300 & $\mathrm{C} 17 \mathrm{~B}-\mathrm{H} 17 \mathrm{~B}$ & 0.9300 \\
\hline $\mathrm{C} 11 \mathrm{~A}-\mathrm{C} 12 \mathrm{~A}$ & $1.379(8)$ & $\mathrm{C} 18 \mathrm{~B}-\mathrm{C} 19 \mathrm{~B}$ & $1.385(8)$ \\
\hline $\mathrm{C} 11 \mathrm{~A}-\mathrm{H} 11 \mathrm{~A}$ & 0.9300 & $\mathrm{C} 18 \mathrm{~B}-\mathrm{H} 18 \mathrm{~B}$ & 0.9300 \\
\hline $\mathrm{C} 12 \mathrm{~A}-\mathrm{C} 13 \mathrm{~A}$ & $1.466(8)$ & $\mathrm{O} 1 \mathrm{C}-\mathrm{C} 3 \mathrm{C}$ & $1.201(12)$ \\
\hline $\mathrm{C} 14 \mathrm{~A}-\mathrm{C} 19 \mathrm{~A}$ & $1.404(9)$ & $\mathrm{N} 1 \mathrm{C}-\mathrm{C} 3 \mathrm{C}$ & $1.379(13)$ \\
\hline $\mathrm{C} 14 \mathrm{~A}-\mathrm{C} 15 \mathrm{~A}$ & $1.407(9)$ & $\mathrm{N} 1 \mathrm{C}-\mathrm{C} 1 \mathrm{C}$ & $1.399(13)$ \\
\hline $\mathrm{C} 15 \mathrm{~A}-\mathrm{C} 16 \mathrm{~A}$ & $1.369(10)$ & $\mathrm{N} 1 \mathrm{C}-\mathrm{C} 2 \mathrm{C}$ & $1.429(13)$ \\
\hline $\mathrm{C} 15 \mathrm{~A}-\mathrm{H} 15 \mathrm{~A}$ & 0.9300 & $\mathrm{C} 1 \mathrm{C}-\mathrm{H} 1 \mathrm{C} 1$ & 0.9600 \\
\hline $\mathrm{C} 16 \mathrm{~A}-\mathrm{C} 17 \mathrm{~A}$ & $1.407(11)$ & $\mathrm{C} 1 \mathrm{C}-\mathrm{H} 1 \mathrm{C} 2$ & 0.9600 \\
\hline $\mathrm{C} 16 \mathrm{~A}-\mathrm{H} 16 \mathrm{~A}$ & 0.9300 & $\mathrm{C} 1 \mathrm{C}-\mathrm{H} 1 \mathrm{C} 3$ & 0.9600 \\
\hline $\mathrm{C} 17 \mathrm{~A}-\mathrm{C} 18 \mathrm{~A}$ & $1.370(10)$ & $\mathrm{C} 2 \mathrm{C}-\mathrm{H} 2 \mathrm{C} 1$ & 0.9600 \\
\hline $\mathrm{C} 17 \mathrm{~A}-\mathrm{H} 17 \mathrm{~A}$ & 0.9300 & $\mathrm{C} 2 \mathrm{C}-\mathrm{H} 2 \mathrm{C} 2$ & 0.9600 \\
\hline $\mathrm{C} 18 \mathrm{~A}-\mathrm{C} 19 \mathrm{~A}$ & $1.402(8)$ & $\mathrm{C} 2 \mathrm{C}-\mathrm{H} 2 \mathrm{C} 3$ & 0.9600 \\
\hline $\mathrm{C} 18 \mathrm{~A}-\mathrm{H} 18 \mathrm{~A}$ & 0.9300 & $\mathrm{C} 3 \mathrm{C}-\mathrm{H} 3 \mathrm{C}$ & 0.9300 \\
\hline $\mathrm{N} 1 \mathrm{~B}-\mathrm{C} 7 \mathrm{~B}$ & $1.336(7)$ & $\mathrm{S} 1-\mathrm{O} 3$ & $1.434(5)$ \\
\hline $\mathrm{N} 1 \mathrm{~B}-\mathrm{C} 1 \mathrm{~B}$ & $1.385(7)$ & $\mathrm{S} 1-\mathrm{O} 1$ & $1.448(5)$ \\
\hline $\mathrm{N} 2 \mathrm{~B}-\mathrm{C} 12 \mathrm{~B}$ & $1.339(7)$ & $\mathrm{S} 1-\mathrm{O} 4$ & $1.451(5)$ \\
\hline $\mathrm{N} 2 \mathrm{~B}-\mathrm{C} 8 \mathrm{~B}$ & $1.372(7)$ & $\mathrm{S} 1-\mathrm{O} 2$ & $1.476(5)$ \\
\hline $\mathrm{N} 3 \mathrm{~B}-\mathrm{C} 13 \mathrm{~B}$ & $1.324(7)$ & & \\
\hline $\mathrm{N} 2 \mathrm{~A}-\mathrm{Ni1}-\mathrm{N} 2 \mathrm{~B}$ & $179.2(2)$ & $\mathrm{C} 8 \mathrm{~B}-\mathrm{N} 2 \mathrm{~B}-\mathrm{Ni1}$ & $119.4(4)$ \\
\hline $\mathrm{N} 2 \mathrm{~A}-\mathrm{Ni1}-\mathrm{N} 3 \mathrm{~A}$ & $77.65(19)$ & $\mathrm{C} 13 \mathrm{~B}-\mathrm{N} 3 \mathrm{~B}-\mathrm{C} 19 \mathrm{~B}$ & $106.3(5)$ \\
\hline $\mathrm{N} 2 \mathrm{~B}-\mathrm{Ni1}-\mathrm{N} 3 \mathrm{~A}$ & $101.70(19)$ & $\mathrm{C} 13 \mathrm{~B}-\mathrm{N} 3 \mathrm{~B}-\mathrm{Ni1}$ & $112.5(4)$ \\
\hline $\mathrm{N} 2 \mathrm{~A}-\mathrm{Ni1}-\mathrm{N} 3 \mathrm{~B}$ & $102.78(19)$ & $\mathrm{C} 19 \mathrm{~B}-\mathrm{N} 3 \mathrm{~B}-\mathrm{Ni} 1$ & $141.3(4)$ \\
\hline $\mathrm{N} 2 \mathrm{~B}-\mathrm{Ni1}-\mathrm{N} 3 \mathrm{~B}$ & $77.59(19)$ & $\mathrm{C} 7 \mathrm{~B}-\mathrm{N} 4 \mathrm{~B}-\mathrm{C} 6 \mathrm{~B}$ & $107.3(5)$ \\
\hline N3A-Ni1-N3B & $91.07(19)$ & $\mathrm{C} 7 \mathrm{~B}-\mathrm{N} 4 \mathrm{~B}-\mathrm{H} 4 \mathrm{BA}$ & 126.3 \\
\hline $\mathrm{N} 2 \mathrm{~A}-\mathrm{Ni1}-\mathrm{N} 1 \mathrm{~B}$ & $101.35(19)$ & $\mathrm{C} 6 \mathrm{~B}-\mathrm{N} 4 \mathrm{~B}-\mathrm{H} 4 \mathrm{BA}$ & 126.3 \\
\hline $\mathrm{N} 2 \mathrm{~B}-\mathrm{Ni1}-\mathrm{N} 1 \mathrm{~B}$ & $78.27(19)$ & $\mathrm{C} 13 \mathrm{~B}-\mathrm{N} 5 \mathrm{~B}-\mathrm{C} 14 \mathrm{~B}$ & $107.1(5)$ \\
\hline N3A-Ni1-N1B & $93.32(19)$ & $\mathrm{C} 13 \mathrm{~B}-\mathrm{N} 5 \mathrm{~B}-\mathrm{H} 5 \mathrm{BA}$ & 126.5 \\
\hline $\mathrm{N} 3 \mathrm{~B}-\mathrm{Ni1}-\mathrm{N} 1 \mathrm{~B}$ & $155.86(18)$ & $\mathrm{C} 14 \mathrm{~B}-\mathrm{N} 5 \mathrm{~B}-\mathrm{H} 5 \mathrm{BA}$ & 126.5 \\
\hline N2A-Ni1-N1A & $77.93(19)$ & N1B $-\mathrm{C} 1 \mathrm{~B}-\mathrm{C} 6 \mathrm{~B}$ & $109.9(5)$ \\
\hline $\mathrm{N} 2 \mathrm{~B}-\mathrm{Ni1}-\mathrm{N} 1 \mathrm{~A}$ & $102.73(19)$ & $\mathrm{N} 1 \mathrm{~B}-\mathrm{C} 1 \mathrm{~B}-\mathrm{C} 2 \mathrm{~B}$ & $130.0(6)$ \\
\hline $\mathrm{N} 3 \mathrm{~A}-\mathrm{Ni1}-\mathrm{N} 1 \mathrm{~A}$ & $155.57(19)$ & $\mathrm{C} 6 \mathrm{~B}-\mathrm{C} 1 \mathrm{~B}-\mathrm{C} 2 \mathrm{~B}$ & $120.0(6)$ \\
\hline $\mathrm{N} 3 \mathrm{~B}-\mathrm{Ni1}-\mathrm{N} 1 \mathrm{~A}$ & $93.53(19)$ & $\mathrm{C} 3 \mathrm{~B}-\mathrm{C} 2 \mathrm{~B}-\mathrm{C} 1 \mathrm{~B}$ & $117.0(7)$ \\
\hline $\mathrm{N} 1 \mathrm{~B}-\mathrm{Ni1}-\mathrm{N} 1 \mathrm{~A}$ & $92.23(19)$ & $\mathrm{C} 3 \mathrm{~B}-\mathrm{C} 2 \mathrm{~B}-\mathrm{H} 2 \mathrm{~B}$ & 121.5 \\
\hline $\mathrm{C} 7 \mathrm{~A}-\mathrm{N} 1 \mathrm{~A}-\mathrm{C} 1 \mathrm{~A}$ & $104.8(5)$ & $\mathrm{C} 1 \mathrm{~B}-\mathrm{C} 2 \mathrm{~B}-\mathrm{H} 2 \mathrm{~B}$ & 121.5 \\
\hline $\mathrm{C} 7 \mathrm{~A}-\mathrm{N} 1 \mathrm{~A}-\mathrm{Ni1}$ & $111.8(4)$ & $\mathrm{C} 2 \mathrm{~B}-\mathrm{C} 3 \mathrm{~B}-\mathrm{C} 4 \mathrm{~B}$ & $122.2(7)$ \\
\hline $\mathrm{C} 1 \mathrm{~A}-\mathrm{N} 1 \mathrm{~A}-\mathrm{Ni1}$ & $143.4(4)$ & $\mathrm{C} 2 \mathrm{~B}-\mathrm{C} 3 \mathrm{~B}-\mathrm{H} 3 \mathrm{~B}$ & 118.9 \\
\hline $\mathrm{C} 8 \mathrm{~A}-\mathrm{N} 2 \mathrm{~A}-\mathrm{C} 12 \mathrm{~A}$ & $119.9(5)$ & $\mathrm{C} 4 \mathrm{~B}-\mathrm{C} 3 \mathrm{~B}-\mathrm{H} 3 \mathrm{~B}$ & 118.9 \\
\hline $\mathrm{C} 8 \mathrm{~A}-\mathrm{N} 2 \mathrm{~A}-\mathrm{Ni} 1$ & $120.0(4)$ & $\mathrm{C} 5 \mathrm{~B}-\mathrm{C} 4 \mathrm{~B}-\mathrm{C} 3 \mathrm{~B}$ & $120.5(7)$ \\
\hline $\mathrm{C} 12 \mathrm{~A}-\mathrm{N} 2 \mathrm{~A}-\mathrm{Ni1}$ & $119.8(4)$ & $\mathrm{C} 5 \mathrm{~B}-\mathrm{C} 4 \mathrm{~B}-\mathrm{H} 4 \mathrm{~B}$ & 119.8 \\
\hline $\mathrm{C} 13 \mathrm{~A}-\mathrm{N} 3 \mathrm{~A}-\mathrm{C} 19 \mathrm{~A}$ & $105.0(5)$ & $\mathrm{C} 3 \mathrm{~B}-\mathrm{C} 4 \mathrm{~B}-\mathrm{H} 4 \mathrm{~B}$ & 119.8 \\
\hline $\mathrm{C} 13 \mathrm{~A}-\mathrm{N} 3 \mathrm{~A}-\mathrm{Ni1}$ & $113.2(4)$ & $\mathrm{C} 4 \mathrm{~B}-\mathrm{C} 5 \mathrm{~B}-\mathrm{C} 6 \mathrm{~B}$ & $118.5(7)$ \\
\hline $\mathrm{C} 19 \mathrm{~A}-\mathrm{N} 3 \mathrm{~A}-\mathrm{Ni1}$ & $141.4(4)$ & $\mathrm{C} 4 \mathrm{~B}-\mathrm{C} 5 \mathrm{~B}-\mathrm{H} 5 \mathrm{~B}$ & 120.7 \\
\hline $\mathrm{C} 7 \mathrm{~A}-\mathrm{N} 4 \mathrm{~A}-\mathrm{C} 6 \mathrm{~A}$ & $106.9(5)$ & $\mathrm{C} 6 \mathrm{~B}-\mathrm{C} 5 \mathrm{~B}-\mathrm{H} 5 \mathrm{~B}$ & 120.7 \\
\hline $\mathrm{C} 7 \mathrm{~A}-\mathrm{N} 4 \mathrm{~A}-\mathrm{H} 4 \mathrm{AA}$ & 126.6 & $\mathrm{~N} 4 \mathrm{~B}-\mathrm{C} 6 \mathrm{~B}-\mathrm{C} 5 \mathrm{~B}$ & $132.8(6)$ \\
\hline $\mathrm{C} 6 \mathrm{~A}-\mathrm{N} 4 \mathrm{~A}-\mathrm{H} 4 \mathrm{AA}$ & 126.6 & $\mathrm{~N} 4 \mathrm{~B}-\mathrm{C} 6 \mathrm{~B}-\mathrm{C} 1 \mathrm{~B}$ & $105.6(5)$ \\
\hline
\end{tabular}




\begin{tabular}{|c|c|}
\hline $\mathrm{C} 13 \mathrm{~A}-\mathrm{N} 5 \mathrm{~A}-\mathrm{C} 14 \mathrm{~A}$ & $106.9(5)$ \\
\hline $\mathrm{C} 13 \mathrm{~A}-\mathrm{N} 5 \mathrm{~A}-\mathrm{H} 5 \mathrm{AA}$ & 126.6 \\
\hline $\mathrm{C} 14 \mathrm{~A}-\mathrm{N} 5 \mathrm{~A}-\mathrm{H} 5 \mathrm{AA}$ & 126.6 \\
\hline $\mathrm{N} 1 \mathrm{~A}-\mathrm{C} 1 \mathrm{~A}-\mathrm{C} 6 \mathrm{~A}$ & $110.3(5)$ \\
\hline $\mathrm{N} 1 \mathrm{~A}-\mathrm{C} 1 \mathrm{~A}-\mathrm{C} 2 \mathrm{~A}$ & $130.4(6)$ \\
\hline $\mathrm{C} 6 \mathrm{~A}-\mathrm{C} 1 \mathrm{~A}-\mathrm{C} 2 \mathrm{~A}$ & $119.4(6)$ \\
\hline $\mathrm{C} 3 \mathrm{~A}-\mathrm{C} 2 \mathrm{~A}-\mathrm{C} 1 \mathrm{~A}$ & $118.7(7)$ \\
\hline $\mathrm{C} 3 \mathrm{~A}-\mathrm{C} 2 \mathrm{~A}-\mathrm{H} 2 \mathrm{~A}$ & 120.6 \\
\hline $\mathrm{C} 1 \mathrm{~A}-\mathrm{C} 2 \mathrm{~A}-\mathrm{H} 2 \mathrm{~A}$ & 120.6 \\
\hline $\mathrm{C} 2 \mathrm{~A}-\mathrm{C} 3 \mathrm{~A}-\mathrm{C} 4 \mathrm{~A}$ & $120.3(7)$ \\
\hline $\mathrm{C} 2 \mathrm{~A}-\mathrm{C} 3 \mathrm{~A}-\mathrm{H} 3 \mathrm{~A}$ & 119.9 \\
\hline $\mathrm{C} 4 \mathrm{~A}-\mathrm{C} 3 \mathrm{~A}-\mathrm{H} 3 \mathrm{~A}$ & 119.9 \\
\hline $\mathrm{C} 5 \mathrm{~A}-\mathrm{C} 4 \mathrm{~A}-\mathrm{C} 3 \mathrm{~A}$ & $122.0(7)$ \\
\hline $\mathrm{C} 5 \mathrm{~A}-\mathrm{C} 4 \mathrm{~A}-\mathrm{H} 4 \mathrm{~A}$ & 119.0 \\
\hline $\mathrm{C} 3 \mathrm{~A}-\mathrm{C} 4 \mathrm{~A}-\mathrm{H} 4 \mathrm{~A}$ & 119.0 \\
\hline $\mathrm{C} 6 \mathrm{~A}-\mathrm{C} 5 \mathrm{~A}-\mathrm{C} 4 \mathrm{~A}$ & $115.9(7)$ \\
\hline $\mathrm{C} 6 \mathrm{~A}-\mathrm{C} 5 \mathrm{~A}-\mathrm{H} 5 \mathrm{~A}$ & 122.1 \\
\hline $\mathrm{C} 4 \mathrm{~A}-\mathrm{C} 5 \mathrm{~A}-\mathrm{H} 5 \mathrm{~A}$ & 122.1 \\
\hline $\mathrm{C} 5 \mathrm{~A}-\mathrm{C} 6 \mathrm{~A}-\mathrm{N} 4 \mathrm{~A}$ & $131.5(6)$ \\
\hline $\mathrm{C} 5 \mathrm{~A}-\mathrm{C} 6 \mathrm{~A}-\mathrm{C} 1 \mathrm{~A}$ & $123.8(7)$ \\
\hline $\mathrm{N} 4 \mathrm{~A}-\mathrm{C} 6 \mathrm{~A}-\mathrm{C} 1 \mathrm{~A}$ & $104.7(5)$ \\
\hline $\mathrm{N} 1 \mathrm{~A}-\mathrm{C} 7 \mathrm{~A}-\mathrm{N} 4 \mathrm{~A}$ & $113.3(5)$ \\
\hline $\mathrm{N} 1 \mathrm{~A}-\mathrm{C} 7 \mathrm{~A}-\mathrm{C} 8 \mathrm{~A}$ & $120.2(5)$ \\
\hline $\mathrm{N} 4 \mathrm{~A}-\mathrm{C} 7 \mathrm{~A}-\mathrm{C} 8 \mathrm{~A}$ & $126.4(5)$ \\
\hline $\mathrm{N} 2 \mathrm{~A}-\mathrm{C} 8 \mathrm{~A}-\mathrm{C} 9 \mathrm{~A}$ & $122.7(5)$ \\
\hline $\mathrm{N} 2 \mathrm{~A}-\mathrm{C} 8 \mathrm{~A}-\mathrm{C} 7 \mathrm{~A}$ & $109.7(5)$ \\
\hline $\mathrm{C} 9 \mathrm{~A}-\mathrm{C} 8 \mathrm{~A}-\mathrm{C} 7 \mathrm{~A}$ & $127.7(5)$ \\
\hline $\mathrm{C} 8 \mathrm{~A}-\mathrm{C} 9 \mathrm{~A}-\mathrm{C} 10 \mathrm{~A}$ & $117.3(6)$ \\
\hline $\mathrm{C} 8 \mathrm{~A}-\mathrm{C} 9 \mathrm{~A}-\mathrm{H} 9 \mathrm{~A}$ & 121.4 \\
\hline $\mathrm{C} 10 \mathrm{~A}-\mathrm{C} 9 \mathrm{~A}-\mathrm{H} 9 \mathrm{~A}$ & 121.4 \\
\hline $\mathrm{C} 11 \mathrm{~A}-\mathrm{C} 10 \mathrm{~A}-\mathrm{C} 9 \mathrm{~A}$ & $120.9(6)$ \\
\hline $\mathrm{C} 11 \mathrm{~A}-\mathrm{C} 10 \mathrm{~A}-\mathrm{H} 10 \mathrm{~A}$ & 119.6 \\
\hline $\mathrm{C} 9 \mathrm{~A}-\mathrm{C} 10 \mathrm{~A}-\mathrm{H} 10 \mathrm{~A}$ & 119.6 \\
\hline $\mathrm{C} 10 \mathrm{~A}-\mathrm{C} 11 \mathrm{~A}-\mathrm{C} 12 \mathrm{~A}$ & $119.5(6)$ \\
\hline $\mathrm{C} 10 \mathrm{~A}-\mathrm{C} 11 \mathrm{~A}-\mathrm{H} 11 \mathrm{~A}$ & 120.2 \\
\hline $\mathrm{C} 12 \mathrm{~A}-\mathrm{C} 11 \mathrm{~A}-\mathrm{H} 11 \mathrm{~A}$ & 120.2 \\
\hline $\mathrm{N} 2 \mathrm{~A}-\mathrm{C} 12 \mathrm{~A}-\mathrm{C} 11 \mathrm{~A}$ & $119.7(5)$ \\
\hline $\mathrm{N} 2 \mathrm{~A}-\mathrm{C} 12 \mathrm{~A}-\mathrm{C} 13 \mathrm{~A}$ & $108.5(5)$ \\
\hline $\mathrm{C} 11 \mathrm{~A}-\mathrm{C} 12 \mathrm{~A}-\mathrm{C} 13 \mathrm{~A}$ & $131.8(6)$ \\
\hline N3A-C13A-N5A & $113.5(5)$ \\
\hline $\mathrm{N} 3 \mathrm{~A}-\mathrm{C} 13 \mathrm{~A}-\mathrm{C} 12 \mathrm{~A}$ & $120.1(6)$ \\
\hline $\mathrm{N} 5 \mathrm{~A}-\mathrm{C} 13 \mathrm{~A}-\mathrm{C} 12 \mathrm{~A}$ & $126.4(5)$ \\
\hline $\mathrm{N} 5 \mathrm{~A}-\mathrm{C} 14 \mathrm{~A}-\mathrm{C} 19 \mathrm{~A}$ & $105.6(5)$ \\
\hline $\mathrm{N} 5 \mathrm{~A}-\mathrm{C} 14 \mathrm{~A}-\mathrm{C} 15 \mathrm{~A}$ & $134.1(7)$ \\
\hline $\mathrm{C} 19 \mathrm{~A}-\mathrm{C} 14 \mathrm{~A}-\mathrm{C} 15 \mathrm{~A}$ & $120.2(6)$ \\
\hline $\mathrm{C} 16 \mathrm{~A}-\mathrm{C} 15 \mathrm{~A}-\mathrm{C} 14 \mathrm{~A}$ & $117.8(8)$ \\
\hline $\mathrm{C} 16 \mathrm{~A}-\mathrm{C} 15 \mathrm{~A}-\mathrm{H} 15 \mathrm{~A}$ & 121.1 \\
\hline $\mathrm{C} 14 \mathrm{~A}-\mathrm{C} 15 \mathrm{~A}-\mathrm{H} 15 \mathrm{~A}$ & 121.1 \\
\hline
\end{tabular}

$\begin{array}{ll} & \\ \text { C5B-C6B-C1B } & 121.6(6) \\ \text { N1B-C7B-N4B } & 113.4(5) \\ \text { N1B-C7B-C8B } & 120.0(5) \\ \text { N4B-C7B-C8B } & 126.7(6) \\ \text { C9B-C8B-N2B } & 121.1(5) \\ \text { C9B-C8B-C7B } & 128.7(6) \\ \text { N2B-C8B-C7B } & 110.2(5) \\ \text { C8B-C9B-C10B } & 118.9(6) \\ \text { C8B-C9B-H9B } & 120.5 \\ \text { C10B-C9B-H9B } & 120.5 \\ \text { C9B-C10B-C11B } & 119.6(6) \\ \text { C9B-C10B-H10B } & 120.2 \\ \text { C11B-C10B-H10B } & 120.2 \\ \text { C12B-C11B-C10B } & 118.6(6) \\ \text { C12B-C11B-H11B } & 120.7 \\ \text { C10B-C11B-H11B } & 120.7 \\ \text { N2B-C12B-C11B } & 120.8(6) \\ \text { N2B-C12B-C13B } & 110.8(5) \\ \text { C11B-C12B-C13B } & 128.4(6) \\ \text { N3B-C13B-N5B } & 112.2(5) \\ \text { N3B-C13B-C12B } & 119.6(5) \\ \text { N5B-C13B-C12B } & 128.2(5) \\ \text { N5B-C14B-C19B } & 107.3(5) \\ \text { N5B-C14B-C15B } & 132.3(6) \\ \text { C19B-C14B-C15B } & 120.4(6) \\ \text { C16B-C15B-C14B } & 117.1(7) \\ \text { C16B-C15B-H15B } & 121.4 \\ \text { C14B-C15B-H15B } & 121.4 \\ \text { C15B-C16B-C17B } & 121.8(7) \\ \text { C15B-C16B-H16B } & 119.1 \\ \text { C17B-C16B-H16B } & 119.1 \\ \text { C18B-C17B-C16B } & 121.6(7) \\ \text { C18B-C17B-H17B } & 119.2 \\ \text { C16B-C17B-H17B } & 119.2 \\ \text { C17B-C18B-C19B } & 117.6(6) \\ \text { C17B-C18B-H18B } & 121.2 \\ \text { C19B-C18B-H18B } & 121.2 \\ \text { C18B-C19B-N3B } & 131.5(6) \\ \text { C18B-C19B-C14B } & 121.3(6) \\ \text { N3B-C19B-C14B } & 107.1(5) \\ \text { C3C-N1C-C1C } & 117.7(11) \\ \text { C3C-N1C-C2C } & 118.0(11) \\ \text { C1C-N1C-C2C } & 124.3(12) \\ \text { N1C-C1C-H1C1 } & 109.5 \\ \text { N1C-C1C-H1C2 } & 109.5 \\ \text { H1C1-C1C-H1C2 } & 109.5 \\ \text { N1C-C1C-H1C3 } & 109.5 \\ \text { H1C1-C1C-H1C3 } & \\ & \end{array}$




$\begin{array}{ll}\mathrm{C} 15 \mathrm{~A}-\mathrm{C} 16 \mathrm{~A}-\mathrm{C} 17 \mathrm{~A} & 122.3(8) \\ \mathrm{C} 15 \mathrm{~A}-\mathrm{C} 16 \mathrm{~A}-\mathrm{H} 16 \mathrm{~A} & 118.9 \\ \mathrm{C} 17 \mathrm{~A}-\mathrm{C} 16 \mathrm{~A}-\mathrm{H} 16 \mathrm{~A} & 118.9 \\ \mathrm{C} 18 \mathrm{~A}-\mathrm{C} 17 \mathrm{~A}-\mathrm{C} 16 \mathrm{~A} & 120.2(8) \\ \mathrm{C} 18 \mathrm{~A}-\mathrm{C} 17 \mathrm{~A}-\mathrm{H} 17 \mathrm{~A} & 119.9 \\ \mathrm{C} 16 \mathrm{~A}-\mathrm{C} 17 \mathrm{~A}-\mathrm{H} 17 \mathrm{~A} & 119.9 \\ \mathrm{C} 17 \mathrm{~A}-\mathrm{C} 18 \mathrm{~A}-\mathrm{C} 19 \mathrm{~A} & 118.9(7) \\ \mathrm{C} 17 \mathrm{~A}-\mathrm{C} 18 \mathrm{~A}-\mathrm{H} 18 \mathrm{~A} & 120.5 \\ \mathrm{C} 19 \mathrm{~A}-\mathrm{C} 18 \mathrm{~A}-\mathrm{H} 18 \mathrm{~A} & 120.5 \\ \text { N3A-C19A-C18A } & 130.4(6) \\ \text { N3A-C19A-C14A } & 109.0(5) \\ \mathrm{C} 18 \mathrm{~A}-\mathrm{C} 19 \mathrm{~A}-\mathrm{C} 14 \mathrm{~A} & 120.6(6) \\ \mathrm{C} 7 \mathrm{~B}-\mathrm{N} 1 \mathrm{~B}-\mathrm{C} 1 \mathrm{~B} & 103.7(5) \\ \mathrm{C} 7 \mathrm{~B}-\mathrm{N} 1 \mathrm{~B}-\mathrm{Ni1} & 112.1(4) \\ \mathrm{C} 1 \mathrm{~B}-\mathrm{N} 1 \mathrm{~B}-\mathrm{Ni1} & 144.1(4) \\ \mathrm{C} 12 \mathrm{~B}-\mathrm{N} 2 \mathrm{~B}-\mathrm{C} 8 \mathrm{~B} & 121.0(5) \\ \mathrm{C} 12 \mathrm{~B}-\mathrm{N} 2 \mathrm{~B}-\mathrm{Ni1} & 119.6(4)\end{array}$

$\mathrm{H} 1 \mathrm{C} 2-\mathrm{C} 1 \mathrm{C}-\mathrm{H} 1 \mathrm{C} 3$

$\mathrm{N} 1 \mathrm{C}-\mathrm{C} 2 \mathrm{C}-\mathrm{H} 2 \mathrm{C} 1$

$\mathrm{N} 1 \mathrm{C}-\mathrm{C} 2 \mathrm{C}-\mathrm{H} 2 \mathrm{C} 2$

$\mathrm{H} 2 \mathrm{C} 1-\mathrm{C} 2 \mathrm{C}-\mathrm{H} 2 \mathrm{C} 2$

$\mathrm{N} 1 \mathrm{C}-\mathrm{C} 2 \mathrm{C}-\mathrm{H} 2 \mathrm{C} 3$

$\mathrm{H} 2 \mathrm{C} 1-\mathrm{C} 2 \mathrm{C}-\mathrm{H} 2 \mathrm{C} 3$

$\mathrm{H} 2 \mathrm{C} 2-\mathrm{C} 2 \mathrm{C}-\mathrm{H} 2 \mathrm{C} 3$

$\mathrm{O} 1 \mathrm{C}-\mathrm{C} 3 \mathrm{C}-\mathrm{N} 1 \mathrm{C}$

$\mathrm{O} 1 \mathrm{C}-\mathrm{C} 3 \mathrm{C}-\mathrm{H} 3 \mathrm{C}$

$\mathrm{N} 1 \mathrm{C}-\mathrm{C} 3 \mathrm{C}-\mathrm{H} 3 \mathrm{C}$

$\mathrm{O} 3-\mathrm{S} 1-\mathrm{O} 1$

$\mathrm{O} 3-\mathrm{S} 1-\mathrm{O} 4$

$\mathrm{O} 1-\mathrm{S} 1-\mathrm{O} 4$

$\mathrm{O} 3-\mathrm{S} 1-\mathrm{O} 2$

$\mathrm{O} 1-\mathrm{S} 1-\mathrm{O} 2$

$\mathrm{O} 4-\mathrm{S} 1-\mathrm{O} 2$
109.5

109.5

109.5

109.5

109.5

109.5

109.5

124.8 (12)

117.6

117.6

106.5 (4)

$114.6(4)$

$108.3(4)$

$109.6(3)$

$110.0(3)$

$107.9(3)$

Hydrogen-bond geometry $\left(\AA,{ }^{\circ}\right)$

\begin{tabular}{lllll}
\hline$D-\mathrm{H} \cdots A$ & $D-\mathrm{H}$ & $\mathrm{H} \cdots A$ & $D \cdots A$ & $D-\mathrm{H} \cdots A$ \\
\hline $\mathrm{N} 4 A-\mathrm{H} 4 A A \cdots \mathrm{O} 4^{\mathrm{i}}$ & 0.86 & 1.89 & $2.732(7)$ & 168 \\
$\mathrm{~N} 4 B-\mathrm{H} 4 B A \cdots \mathrm{S} 1^{\mathrm{ii}}$ & 0.86 & 2.73 & $3.514(5)$ & 152 \\
$\mathrm{~N} 4 B-\mathrm{H} 4 B A \cdots \mathrm{O} 2^{\mathrm{ii}}$ & 0.86 & 1.79 & $2.646(7)$ & 176
\end{tabular}

Symmetry codes: (i) $-x+2,-y+1,-z+1$; (ii) $-x+3 / 2, y, z-1 / 2$. 